\title{
Young Stellar Object Variability (YSOVAR): Long Timescale Variations in the Mid-Infrared
}

\author{
L. M. Rebull
}

A. M. Cody

K. R. Covey

H. M. Gunther

L. A. Hillenbrand

See next page for additional authors

Follow this and additional works at: https://bearworks.missouristate.edu/articles-cnas

\section{Recommended Citation}

Rebull, L. M., A. M. Cody, K. R. Covey, H. M. GÃ²/4nther, L. A. Hillenbrand, P. Plavchan, K. Poppenhaeger et al. "Young Stellar Object Variability (YSOVAR): Long Timescale Variations in the Mid-Infrared." The Astronomical Journal 148, no. 5 (2014): 92.

This article or document was made available through BearWorks, the institutional repository of Missouri State University. The work contained in it may be protected by copyright and require permission of the copyright holder for reuse or redistribution.

For more information, please contact BearWorks@library.missouristate.edu. 


\section{Authors}

L. M. Rebull; A. M. Cody; K. R. Covey; H. M. Gunther; L. A. Hillenbrand; P. Plavchan; K. Poppenhaeger; J. R. Stauffer; S. J. Wolk; and For complete list of authors, see publisher's website. 


\title{
YOUNG STELLAR OBJECT VARIABILITY (YSOVAR): LONG TIMESCALE VARIATIONS IN THE MID-INFRARED
}

\author{
L. M. Rebull ${ }^{1}$, A. M. Cody ${ }^{1}$, K. R. Cover $^{2}$, H. M. Günther ${ }^{3}$, L. A. Hillenbrand ${ }^{4}$, P. Plavehan ${ }^{5,6}$, K. Poppenhaeger $^{3,27}$, \\ J. R. Stauffer ${ }^{1}$, S. J. Wolk ${ }^{3}$, R. Gutermuth ${ }^{7}$, M. Morales-Calderón ${ }^{1,8}$, I. Song ${ }^{9}$, D. Barrado ${ }^{8}$, A. Bayo ${ }^{10,11}$, D. James ${ }^{12}$, \\ J. L. Hora ${ }^{3}$, F. J. Vrba ${ }^{13}$, C. Alves de Oliveira ${ }^{14}$, J. Bouvier ${ }^{15}, 16$, S. J. Carey ${ }^{1}$, J. M. Carpenter ${ }^{4}$, F. Favata ${ }^{17}$, \\ K. Flaherty ${ }^{18}$, J. Forbrich ${ }^{3,19}$, J. Hernandez ${ }^{20}$, M. J. McCaughrean ${ }^{17}$, S. T. Megeath ${ }^{21}$, G. Micela $^{22}$, H. A. Smith $^{3}$, \\ S. Terebey ${ }^{23}$, N. Turner ${ }^{24}$, L. Allen ${ }^{25}$, D. Ardila ${ }^{26}$, H. Bour ${ }^{8}$, And S. Guieu ${ }^{15}$ \\ ${ }^{1}$ Spitzer Science Center (SSC), Infrared Processing and Analysis Center (IPAC), 1200 East California Boulevard, \\ California Institute of Technology, Pasadena, CA 91125, USA; rebull@ipac.caltech.edu \\ ${ }^{2}$ Lowell Observatory, 1400 West Mars Hill Road, Flagstaff, AZ 86001, USA \\ ${ }^{3}$ Harvard-Smithsonian Center for Astrophysics, 60 Garden Street, Cambridge, MA 02138, USA \\ ${ }^{4}$ Department of Astronomy, California Institute of Technology, Pasadena, CA 91125, USA \\ ${ }^{5}$ NASA Exoplanet Science Institute (NExScI), Infrared Processing and Analysis Center (IPAC), 1200 East California Boulevard, \\ California Institute of Technology, Pasadena, CA 91125, USA \\ ${ }^{6}$ Missouri State University, 901 South National Avenue, Springfield, MO 65897, USA \\ ${ }^{7}$ Department of Astronomy, University of Massachusetts, Amherst, MA 01003, USA \\ ${ }^{8}$ Departamento de Astrofísica, Centro de Astrobiología (INTA-CSIC), ESAC campus, P.O. Box 78, E-28691 Villanueva de la Cañada, Spain \\ ${ }^{9}$ Physics and Astronomy Department, University of Georgia, Athens, GA 30602-2451, USA \\ ${ }^{10}$ Max Planck Institut für Astronomie, Königstuhl 17, D-69117, Heidelberg, Germany \\ ${ }^{11}$ Departamento de Física y Astronomía, Facultad de Ciencias, Universidad de Valparaíso, Av. Gran Bretaña 1111, 5030 Casilla, Valparaíso, Chile \\ ${ }^{12}$ Cerro Tololo InterAmerican Observatory (CTIO), Casilla 603, La Serena, Chile \\ ${ }^{13}$ US Naval Observatory, Flagstaff Station 10391 West Naval Observatory Road, Flagstaff, AZ 86005, USA \\ ${ }^{14}$ European Space Agency (ESA/ESAC), P.O. Box 78, E-28691 Villanueva de la Caãda, Madrid, Spain \\ ${ }^{15}$ Univ. Grenoble Alpes, IPAG, F-38000 Grenoble, France \\ ${ }^{16}$ CNRS, IPAG, F-38000 Grenoble, France \\ ${ }^{17}$ European Space Agency, ESTEC, Postbus 299, 2200 AG Noordwijk, The Netherlands \\ ${ }^{18}$ Astronomy Department, Wesleyan University, 96 Foss Hill Dr., Middletown, CT 06459, USA \\ ${ }^{19}$ Department of Astrophysics, University of Vienna, Türkenschanzstr. 17, A-1180 Vienna, Austria \\ ${ }^{20}$ Centro de Investigaciones de Astronomía, Apdo. Postal 264, Mérida 5101-A, Venezuela \\ ${ }^{21}$ Ritter Astrophysical Observatory, Department of Physics and Astronomy, University of Toledo, Toledo, OH 43606, USA \\ ${ }^{22}$ INAF-Osservatorio Astronomico di Palermo, Piazza del Parlamento 1, I-90134 Palermo, Italy \\ ${ }^{23}$ Department of Physics and Astronomy, 5151 State University Drive, California State University at Los Angeles, Los Angeles, CA 90032, USA \\ ${ }^{24}$ Jet Propulsion Laboratory, 4800 Oak Grove Drive, Pasadena, CA 91109, USA \\ ${ }^{25}$ NOAO, 950 North Cherry Avenue, Tucson, AZ, USA \\ ${ }^{26}$ NASA Herschel Science Center (NHSC), Infrared Processing and Analysis Center (IPAC), 1200 East California Boulevard, \\ California Institute of Technology, Pasadena, CA 91125, USA \\ Received 2014 June 3; accepted 2014 August 27; published 2014 October 14
}

\begin{abstract}
The YSOVAR (Young Stellar Object VARiability) Spitzer Space Telescope observing program obtained the first extensive mid-infrared (3.6 and $4.5 \mu \mathrm{m})$ time series photometry of the Orion Nebula Cluster plus smaller footprints in 11 other star-forming cores (AFGL 490, NGC 1333, Mon R2, GGD 12-15, NGC 2264, L1688, Serpens Main, Serpens South, IRAS 20050+2720, IC 1396A, and Ceph C). There are 29,000 unique objects with light curves in either or both IRAC channels in the YSOVAR data set. We present the data collection and reduction for the Spitzer and ancillary data, and define the "standard sample" on which we calculate statistics, consisting of fast cadence data, with epochs roughly twice per day for $\sim 40$ days. We also define a "standard sample of members" consisting of all the IR-selected members and X-ray-selected members. We characterize the standard sample in terms of other properties, such as spectral energy distribution shape. We use three mechanisms to identify variables in the fast cadence data-the Stetson index, a $\chi^{2}$ fit to a flat light curve, and significant periodicity. We also identified variables on the longest timescales possible of six to seven years by comparing measurements taken early in the Spitzer mission with the mean from our YSOVAR campaign. The fraction of members in each cluster that are variable on these longest timescales is a function of the ratio of Class I/total members in each cluster, such that clusters with a higher fraction of Class I objects also have a higher fraction of long-term variables. For objects with a YSOVAR-determined period and a [3.6]-[8] color, we find that a star with a longer period is more likely than those with shorter periods to have an IR excess. We do not find any evidence for variability that causes [3.6]-[4.5] excesses to appear or vanish within our data set; out of members and field objects combined, at most $0.02 \%$ may have transient IR excesses.
\end{abstract}

Key words: circumstellar matter - stars: pre-main sequence - stars: protostars - stars: variables: general

Online-only material: color figures 


\section{INTRODUCTION}

Optical variability was one of the original defining characteristics of the class of object later determined to be stars in the process of formation (Joy 1945; Herbig 1952), or young stellar objects (YSOs). Optical and near-infrared (NIR) monitoring over timescales of weeks to months of the nearest star-forming regions (SFRs) have shown that the surfaces of YSOs are often mottled, with both hot spots (where gas accretion columns from the inner disk impact the stellar surface) and cool spots (starspots analogous to sunspots; Rydgren \& Vrba 1983; Vrba et al. 1986; Bouvier et al. 1993). Because the stars are also rotating, the presence of spots causes their apparent luminosities and colors to vary with the stellar rotation period. As summarized in Herbst et al. (1994), cool spots are found on YSOs without disks, or at least without substantial accretion disks (weak-lined T Tauri stars, or WTTs), which is expected since those stars do not generally have other signatures of active accretion; however, both cool and hot spots have been identified on YSOs with substantial disks (classical T Tauri stars, or CTTs). The largest amplitude, most variable optical light curves are generally attributed to hot spots (Vrba et al. 1993).

The periodicities found in spot-dominated light curves have been taken to be the rotation period of the star, and the derivation of periods has long been the most common analysis of time series data of young stars. For solar mass YSOs and ages few Myr, the distribution of rotational velocities is bimodal, with one set of stars having periods on the order of 2-4 days and the other with characteristic periods of 8-12 days (e.g., Cieza \& Baliber 2007, and references therein). This period distribution has been interpreted in terms of a model where the rotational periods of the accreting stars are magnetically locked to the Keplerian rotation period of their inner disks (with periods on the order of 10 days), whereas stars that are no longer accreting spin up as they contract, thus associating the short-period peak in the rotation period distribution with stars that have lost their disks at young ages (e.g., Bouvier et al. 1997). This correlation at young ages appears to persist to later ages, with the slow rotators on the zero-age main sequence (the stars with longlived accretion disks) being more likely to have debris disks, which could suggest that these slow rotators are more likely to have formed planets (see, e.g., Bouvier 2008; McQuillan et al. 2013a, 2013b).

In the past, periodicities have been most frequently determined using ground-based optical time series observations. Optical observations are primarily sensitive to phenomena associated with the stellar photosphere or with other energetically "hot" regions (hot spots, accretion columns, chromospheres), and are limited in regions of high extinction. In contrast, observations at longer wavelengths penetrate extinction and also offer a new perspective by being sensitive to variability associated with "warm" or "cool" regions-the disks and envelopes of YSOs. The dominant contributions to YSO photometric variability in the IR include the Rayleigh-Jeans tail of the hotter processes, as well as dust reprocessing of emission from these hotter processes, along with phenomena uniquely associated with the disk. Relevant disk processes might involve thermal emission from an overdense (or overwarmed) region of the inner disk, variable disk accretion, structure in the disk rotating into and out of view causing changes in the measured $A_{V}$ toward the star, or disk instabilities (e.g., Fedele et al. 2007; Plavchan

\footnotetext{
27 NASA Sagan Fellow.
}

et al. 2008a, 2013; Herbst et al. 2010). Finally, standard geometric effects due to orbiting companions can also be probed in the mid-infrared, uniquely so for more embedded sources. Because many more physical processes can affect the variability of YSOs in the infrared, relatively few infrared light curves are periodic and thus straightforward to analyze (see, e.g., Cody et al. 2014; Morales-Calderón et al. 2011).

One of the first monitoring programs of YSOs at NIR wavelengths was Skrutskie et al. (1996), which monitored 15 YSOs in Taurus-Auriga. They found periodic variability, as well as variability due to accretion and extinction.

The first large program of time series photometry of YSOs at wavelengths longward of 1 micron was by Carpenter et al. (2001, hereafter CHS01). CHS01 obtained $J H K_{s}$ monitoring of $\sim 3 \mathrm{deg}^{2}$ of the Orion Nebula Cluster (ONC) over a $\sim 1$ month time period as part of the Two-Micron All Sky Survey (2MASS; Skrutskie et al. 2006). About 1000 Orion members showed NIR photometric variability in their data. ${ }^{28}$ Typical light curve amplitudes were of the order of $0.2 \mathrm{mag}$, but with some stars exhibiting amplitudes up to 2 mag; periods were determined for about a quarter of their stars. CHS01 attributed the variability for somewhat more than half of the stars to cool spots; they suspected that most of the others could be explained by hot spots, variable extinction, or variable accretion. However, they could not make a definitive determination and suggested multiple mechanisms could be involved. 2MASS monitored other SFRs as well, including Chamaeleon and Rho Oph. Carpenter et al. (2002) reported on the more limited 2MASS study of the Chamaeleon SFR and similarly characterized variability amplitudes and behaviors, along with identifying new candidate young star members via their infrared variability. Plavchan et al. (2008b) and Parks et al. (2014) report on the 2MASS observations of a small region in Rho Oph, finding about 100 variables with roughly similar variability properties as Orion in that the amplitude variations were found to be between a few tenths and $2 \mathrm{mag}$, with periods obtained for about onethird of the sample. Subsequent to 2MASS, more recently, there have been a number of NIR monitoring programs studying other SFRs, such as Wolk et al. (2013), which monitored Cyg OB7, finding several classes of YSO NIR variability.

In the mid-infrared (Cohen \& Schwartz 1976), as for the nearinfrared (e.g., Elias et al. 1978; Rydgren \& Vrba 1983), previous literature suggested at least small variations on timescales of months to years, likely attributable to circumstellar disk processes. However, in the same way that charge-coupled devices (CCDs) revolutionized our ability to discern precisely optical variability trends and 2MASS did the same for nearinfrared variability, the Spitzer Space Telescope (Werner et al. 2004) has allowed us to probe even small variations in the midinfrared; Spitzer is a photometrically stable (better than 1\%), sensitive, wide-field $\left(5^{\prime} \times 5^{\prime}\right)$, Earth-trailing (avoiding orbital day/night aliasing) platform. Spitzer, specifically the Infrared Array Camera (IRAC; Fazio et al. 2004), observes at bands sensitive to both YSO photospheres and circumstellar dust.

Cycle 6 was the first post-cryogen Spitzer cycle, using only IRAC's first two channels (3.6 and $4.5 \mu \mathrm{m}$, often abbreviated as IRAC-1 and IRAC-2, or I1 and I2; when reporting measurements in magnitudes, the bands are written with brackets, e.g., [3.6] = $16.38 \mathrm{mag}$ ). The YSOVAR (Young Stellar Object VARiability) the Spitzer Space Telescope Cycle-6 Exploration Science (ES) Program was approved for $550 \mathrm{hr}$ of observations,

28 http://www.astro.caltech.edu/ jmc/variables/orion 
Table 1

Summary of Cluster Properties

\begin{tabular}{|c|c|c|c|c|c|c|}
\hline Cluster $^{\mathrm{a}}$ & $\begin{array}{l}\text { Dist. } \\
(\mathrm{pc})\end{array}$ & Gal. Lat. $^{b}$ & $\begin{array}{l}\text { Class II/I } \\
(\mathrm{G} 09)^{\mathrm{c}}\end{array}$ & $\begin{array}{c}\text { Class II/I } \\
(\text { Obj. w/L.C. })^{\mathrm{d}}\end{array}$ & $\begin{array}{c}\text { Class I/tot } \\
(\text { Mem. w/L.C. })^{\mathrm{e}}\end{array}$ & Notes \\
\hline AFGL 490 & 900 & +1.8 & $\sim 3.2$ & $4.5 \pm 0.9$ & $0.20 \pm 0.04$ & $\begin{array}{l}\text { Distance: Testi et al. (1998); ratio: Masiunas et al. } \\
\text { (2012), using the same approach as G09 but deeper } \\
\text { data, report a ratio of } \sim 5\end{array}$ \\
\hline NGC 1333 & 235 & -20.5 & $\sim 2.7$ & $4.7 \pm 1.3$ & $0.16 \pm 0.04$ & $\begin{array}{l}\text { Distance: Hirota et al. (2008); see also Hirota et al. } \\
\text { (2011) }\end{array}$ \\
\hline Orion & 414 & -19.0 & $\cdots$ & $9.7 \pm 0.9$ & $0.07 \pm 0.01$ & Distance: Menten et al. (2007) \\
\hline Mon R2 & 830 & -12.6 & $\sim 4.7$ & $6.0 \pm 1.4$ & $0.15 \pm 0.03$ & $\begin{array}{l}\text { Distance: Herbst \& Racine (1976); see discussion in } \\
\text { Carpenter \& Hodapp (2008) }\end{array}$ \\
\hline GGD 12-15 & 830 & -11.9 & $\sim 4.2$ & $5.8 \pm 1.6$ & $0.13 \pm 0.03$ & $\begin{array}{l}\text { Distance: Herbst \& Racine (1976); see discussion in } \\
\text { Carpenter \& Hodapp (2008) }\end{array}$ \\
\hline NGC 2264 & 760 & +2.1 & $\cdots$ & $3.0 \pm 0.6$ & $0.15 \pm 0.03$ & Distance: Sung et al. (1997) \\
\hline L1688 & 120 & +16.6 & $\sim 3.0$ & $1.9 \pm 0.6$ & $0.28 \pm 0.08$ & $\begin{array}{l}\text { Distance: Wilking et al. (2008), Loinard et al. } \\
\text { (2013), Loinard et al. (2008); G09 cites Wilking } \\
\text { et al. (2005) for } 150 \mathrm{pc}\end{array}$ \\
\hline Serpens Main & 415 & +16.5 & $\sim 1.4$ & $2.2 \pm 0.6$ & $0.21 \pm 0.05$ & $\begin{array}{l}\text { Distance: Dzib et al. (2010), Loinard et al. (2013) } \\
\text { (see also Eiroa et al. 2008); G09, as do many other } \\
\text { authors, cites } 260 \text { pc from Straižys et al. (1996) }\end{array}$ \\
\hline Serpens South & 415 & +3.8 & $\sim 0.7$ & $0.9 \pm 0.2$ & $0.41 \pm 0.08$ & $\begin{array}{l}\text { G09 ratio calculated from numbers in Gutermuth } \\
\text { et al. }(2008 b) ; \text { assumed to be same distance as } \\
\text { Serpens Main }\end{array}$ \\
\hline IRAS $20050+2720$ & 700 & -2.6 & $\sim 1.9$ & $2.2 \pm 0.4$ & $0.30 \pm 0.05$ & Distance: Wilking et al. (1989), Günther et al. (2012) \\
\hline IC 1396A & 900 & +3.9 & $\cdots$ & $7.9 \pm 2.8$ & $0.04 \pm 0.01$ & $\begin{array}{l}\text { Distance: Contreras et al. ( } 2002) \text {; II/I ratio given is } \\
\text { in region with both } 11 \text { and } 2 \text { Light curves-II/I } \\
\text { ratio calculated in exactly the same way as other } \\
\text { clusters is } 11.6 \pm 4.0\end{array}$ \\
\hline Ceph C & 700 & +2.1 & $\sim 2.3$ & $3.2 \pm 1.0$ & $0.17 \pm 0.05$ & $\begin{array}{l}\text { Distance: Moscadelli et al. (2009); II/I ratio given is } \\
\text { in region with both } \mathrm{I} 1 \text { and } \mathrm{I} 2 \text { light curves-II/I ratio } \\
\text { calculated in exactly the same way as other clusters } \\
\text { is } 3.8 \pm 1.1\end{array}$ \\
\hline
\end{tabular}

Notes.

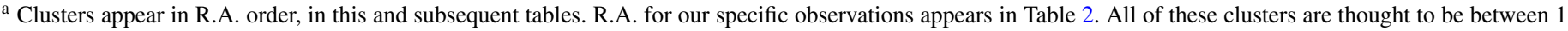
and 5 Myr old.

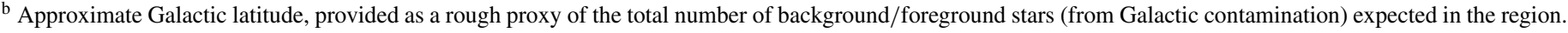

c Ratio of Class II to Class I sources, as presented in G09. For a brief definition of SED classes, see Appendix B.

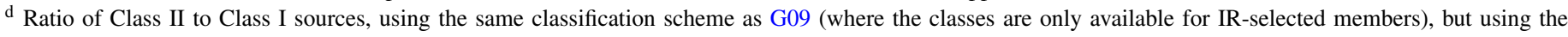

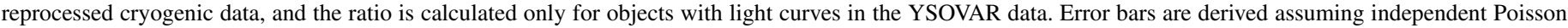
uncertainties on the numbers used to compute the ratio. See Section 4.1 for further discussion.

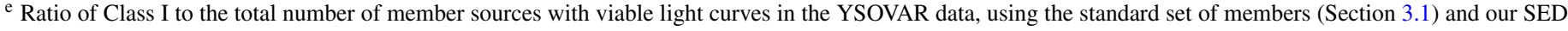

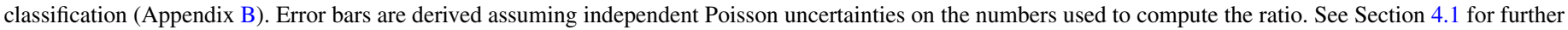
discussion on this ratio and how it compares to the Class II/Class I ratio.

with the goal of obtaining the first extensive mid-infrared time series photometry of the central $\sim 1^{\circ}$ of the ONC plus smaller footprints in 11 other star-forming cores; see Table 1 for a list of the clusters. There are several other ES and smaller programs exploring YSOs in the time domain with Spitzer, many of which are affiliated (to varying degrees) with the larger YSOVAR effort. We have incorporated under the YSOVAR umbrella some additional strongly related programs pre-dating and arising from YSOVAR, resulting in a total of $786 \mathrm{hr}$ of Spitzer time. About $130 \mathrm{hr}$ of that is dedicated observations of NGC 2264 (Coordinated Synoptic Investigation: NGC 2264, or CSI 2264), which is discussed by Cody et al. (2014) and others (e.g., Stauffer et al. 2014; Stauffer et al. 2015, in preparation). CSI 2264 is not discussed in the same way as the rest of the data here, in no small part because the observations are generally different and because it involves the coordination of additional telescopes. A list of core YSOVAR programs and affiliated programs is presented in Table 2 and discussed in Section 2.2. We sometimes refer to the components of the original YSOVAR program as "YSOVAR-classic" to distinguish them from the smaller affil- iated observations obtained over the same time period. There are $\sim 29,000$ unique objects with light curves from either (or both) of the IRAC channels in the YSOVAR data set, which are matched to $\sim 39,000$ individual light curves. These light curve counts include light curves from both cluster members and a significant number of non-member stars, and also likely include extragalactic objects. There are more light curves than objects because most objects have light curves at only I1 or I2, but many have light curves at both I1 and I2.

YSOVAR data were obtained to help reveal the structure of the inner disk region of YSOs, provide new constraints on accretion and extinction variability, assess timescales of mid-IR variability from seconds to years, identify new young eclipsing binaries, help identify new very low-mass substellar members of the surveyed clusters, constrain the short- and long-term stability of hot spots on the surfaces of YSOs, and determine rotational periods for objects too embedded for such monitoring in the optical.

In this paper, in addition to presenting an overview of the data set, one of our goals is to specifically address the longest 
Table 2

Summary of YSOVAR (and Closely Related) Spitzer Observations

\begin{tabular}{|c|c|c|c|c|c|c|c|c|c|c|}
\hline Cluster & $\mathrm{PID}^{\mathrm{a}}$ & $\mathrm{PI}^{\mathrm{b}}$ & $\begin{array}{l}\text { Approx. Obs. } \\
\text { Center (J2000) }\end{array}$ & $\begin{array}{l}\text { Approx. } \\
\text { Ecl.Lat. }\end{array}$ & Date Observed & Epochs & Cadence $^{\mathrm{c}}$ & $\begin{array}{l}\text { No. Obj } \\
\text { w/LCs }\end{array}$ & $\begin{array}{l}\text { Total Exp. } \\
\text { Time (hr) }\end{array}$ & Notes \\
\hline AFGL 490 & 60014 & J. R. Stauffer & $03: 27: 24+58: 44: 00$ & +38 & 2011 Oct-Nov & 46 & Fast & $\sim 1970$ & 9.1 & 2 IRAC FOVs \\
\hline NGC 1333 & 61026 & J. R. Stauffer & $03: 29: 06+31: 19: 30$ & +12 & 2011 Oct-Nov & 73 & Fast & $\sim 690$ & 18.5 & $2 \times 2$ IRAC FOVs \\
\hline Orion & 61028 & J. R. Stauffer & $05: 35: 15-05: 21: 00$ & -28 & $\begin{array}{l}2009 \text { Oct-Dec; } \\
2010 \text { Oct-Dec }\end{array}$ & 80 & Fast & $\sim 7500$ & 365.2 & $\begin{array}{l}\text { very large IRAC map }(\sim 0.9 \\
\text { sq. deg.) }\end{array}$ \\
\hline Orion & 61028 & J. R. Stauffer & $05: 35: 27-04: 47: 31$ & -28 & 2011 Nov & $\sim 270$ & Staring & $\sim 180$ & 10 & staring eclipsing binary follow-up \\
\hline Orion & 61028 & J. R. Stauffer & $05: 35: 27-04: 47: 31$ & -28 & 2011 Nov & 94 & $\mathrm{~EB}$ & $\sim 480$ & 14.7 & 2 FOVs, mapping; EB follow-up \\
\hline Orion & 70025 & J. R. Stauffer & $05: 35: 02-05: 18: 30$ & -28 & 2010 Nov-Dec & 74 & $\sim$ Hourly & e & 30.3 & Dipper follow-up \\
\hline Mon R2 & 61025 & J. R. Stauffer & $06: 07: 48-06: 25: 00$ & -30 & 2010 Nov-Dec & 46 & Fast & $\sim 710$ & 5.6 & 1 IRAC FOV \\
\hline GGD 12-15 & 61021 & J. R. Stauffer & $06: 10: 48-06: 12: 30$ & -30 & 2010 Nov-Dec & 77 & Fast & $\sim 1010$ & 14.8 & 2 IRAC FOVs \\
\hline GGD 12-15 & 70172 & J. Forbrich & $06: 10: 48-06: 12: 30$ & -30 & $2010 \mathrm{Dec}$ & $\sim 530$ & Staring & $\sim 370$ & 20.0 & $\begin{array}{l}1 \text { staring FOV; simul. w/CXO, \& } \\
\text { YSOVAR-like 2-field obs. at } \\
\text { start/end }\end{array}$ \\
\hline NGC 2264 & 61027 & J. R. Stauffer & $06: 41: 04+09: 35: 10$ & -13 & 2010 Nov-Dec & 39 & Fast & $\sim 780$ & 4.7 & one IRAC FOV \\
\hline NGC 2264 & 80040 & J. R. Stauffer & $06: 40: 48+09: 42: 00$ & -13 & $2011 \mathrm{Dec}$ & f & CSI & $\sim 16,500$ & 99.1 & $\begin{array}{l}\text { Cy8-CSI 2264, staring \& } \\
\text { mapping }\end{array}$ \\
\hline NGC 2264 & 90098 & J. R. Stauffer & $06: 40: 48+09: 42: 00$ & -13 & $\begin{array}{l}2013 \text { Dec-2014 } \\
\text { Jan }\end{array}$ & 80 & CSI & $\sim 4700$ & 30.3 & $\begin{array}{l}\text { Cy } 9-\text { CSI } 2264 \text { followup on } \sim 15 \\
\text { targets (cluster targets) }\end{array}$ \\
\hline L1688 & 61024 & J. R. Stauffer & $16: 27: 10-24: 37: 30$ & -3 & $\begin{array}{l}2010 \text { Apr-May; } \\
2010 \text { Sep-Oct; } \\
2011 \text { Apr-May; } \\
2011 \text { Oct-Nov }\end{array}$ & 108 & Fast/slow & $\sim 840$ & 30.7 & $\begin{array}{l}3 \text { IRAC FOVs, fast cadence } 2010 \\
\text { Apr-May }\end{array}$ \\
\hline L1688 & 60109 & P. Plavchan & $16: 27: 31-24: 40: 45$ & -3 & $2010 \mathrm{Apr}$ & $\sim 2500$ & Staring & $\sim 90$ & 24.0 & reverberation mapping \\
\hline L1688 & 90128 & H. M. Günther & $16: 27: 31-24: 40: 45$ & -3 & 2013 May-Jun & 10 & 3-4 days & $\sim 840$ & 2.8 & Cy9 follow-up \\
\hline Serpens Main & 30319 & G. Fazio & $18: 29: 59+01: 13: 53$ & +24 & 2006 Sep & 29 & Cryo & $\sim 2800$ & 6.5 & $\begin{array}{l}\text { cryo obs; same map as PC; } 9 \text { more } \\
\text { hrs staring obtained on subset }\end{array}$ \\
\hline Serpens Main & 61029 & J. R. Stauffer & $18: 29: 59+01: 13: 53$ & +24 & 2011 May-Jun & 82 & Fast & $\sim 3400$ & 16.2 & 2 IRAC FOVs \\
\hline Serpens South & 61030 & J. R. Stauffer & $18: 30: 04-02: 02: 05$ & +21 & 2011 May-Jun & 82 & Fast & $\sim 1540$ & 10.0 & 1 IRAC FOV \\
\hline IRAS $20050+2720$ & 61023 & J. R. Stauffer & $20: 07: 04+27: 29: 14$ & +46 & 2010 Jun-Aug & 102 & Fast & $\sim 3030$ & 13.4 & 1 IRAC FOV \\
\hline IC $1396 \mathrm{~A}$ & 470 & B. T. Soifer & $21: 36: 30+57: 29: 48$ & +64 & 2008 Jan-Feb & 29 & Cryo & $\sim 4500$ & 15.0 & $\begin{array}{l}2 \times 3 \text { IRAC FOVs; DDT; see } \\
\text { Morales-Calderón et al. (2009) }\end{array}$ \\
\hline IC $1396 \mathrm{~A}$ & 497 & J. R. Stauffer & $21: 36: 45+57: 30: 20$ & +64 & 2008 Dec & 10 & Cryo & $\sim 1800$ & 1.5 & $\begin{array}{l}1 \text { IRAC FOV; DDT; } 16 \\
\sim \text { contemporaneous MIPS } 24 \mu \mathrm{m} \\
\text { epochs obtained, } 2.1 \text { more hrs. }\end{array}$ \\
\hline IC $1396 \mathrm{~A}$ & 61022 & J. R. Stauffer & $21: 36: 30+57: 29: 48$ & +64 & $\begin{array}{l}2009 \text { Aug-2010 } \\
\text { Mar; } 2010 \\
\text { Aug-2011 Feb }\end{array}$ & $143^{g}$ & Fast/slow & $\sim 5100$ & 38.9 & $\begin{array}{l}2 \times 3 \text { IRAC FOVs; fast cadence } \\
2009 \text { Sep-Nov }\end{array}$ \\
\hline Ceph C & 61020 & J. R. Stauffer & $23: 05: 51+62: 30: 55$ & +59 & $\begin{array}{l}2009 \text { Dec-2010 } \\
\text { Mar; } 2011 \\
\text { Jan-Mar; } 2011 \\
\text { Sep-Nov }\end{array}$ & 39 & Fast/slow & $\sim 1950$ & 4.8 & $\begin{array}{l}1 \text { IRAC FOV; fast cadence } 2010 \\
\text { Aug-Nov }\end{array}$ \\
\hline$($ Ceph C) & $(61020)$ & (K. Covey) & $23: 05: 51+62: 30: 55$ & +59 & 2010 Aug-Nov & 105 & Fast & (as above) & 13.5 & $\begin{array}{l}1 \mathrm{FOV} \text {; simul. w/CXO (CXO } \\
\text { Cy11 P.I.:K. Covey); AORs } \\
\text { entirely included within program } \\
61020\end{array}$ \\
\hline
\end{tabular}

Notes.

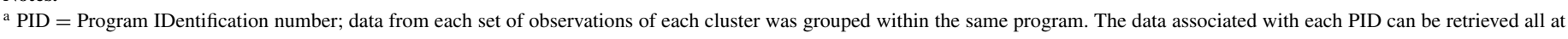
once from the Spitzer Heritage Archive using this number. YSOVAR-classic programs are 60014 and 61020-61030, inclusive.

b PI = Program's Primary Investigator.

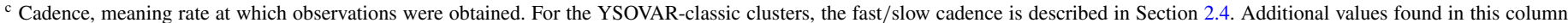

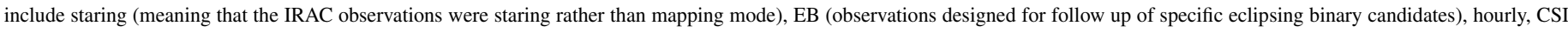
(cadence described in Cody et al. 2014), cryo (observations conducted in cryogenic era and cadence was different for each program).

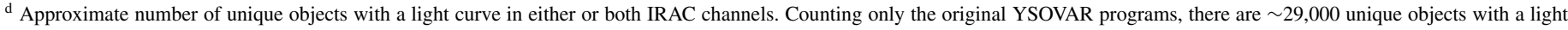

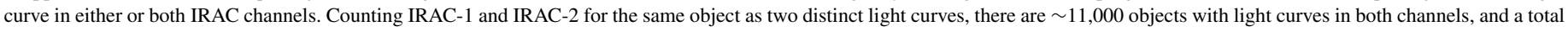
of $\sim 39,000$ light curves.

${ }^{\mathrm{e}}$ Counted as part of light curves listed for program 61028.

f 344 AORs used in this program.

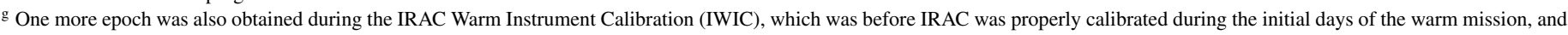
so that epoch is not included in our final data set.

timescale variations that we can quantify in these clusters, six to seven years. We look for large changes between the earliest Spitzer observations (from observations obtained early in the cryogenic era) and the YSOVAR monitoring observations. We discuss many of the technical details associated with the data reduction across the YSOVAR effort. The Orion data were first described by Morales-Calderón et al. (2011, hereafter MC11). The other 11 smaller-field clusters are introduced in this paper, but will be discussed in detail in other papers (the first of which, on L1688, is Günther et al. 2014). Here, we first provide a summary of the observations and data reduction (Section 2), followed by a definition of the samples we use (Section 3). We present some global statistics on all the clusters in Section 4. We delve into variable selection in Section 5, discussing different tests for selecting variables and simulating the sensitivity of the techniques given the actual data set. We present some analysis that is best performed with all the clusters together in Section 6, such as fractions of long-term variables across clusters, correlations between rotation rate and IR excess, and the absence of transient disks. We summarize in Section 7. 
For completeness, we note here that we refer to the 12 regions of recent star formation that we observed for YSOVAR as "clusters," knowing that others may prefer "associations" or other nomenclature. Our targets resemble small condensations within a region; Gutermuth et al. (2009), among others, addresses formal clustering in these regions.

\section{OBSERVATIONS AND DATA REDUCTION}

In this section, we review the target selection and some general properties of the clusters, and describe the Warm Spitzer observations: observing strategy, data reduction, cadence, and the noise floor. We also describe the data reduction for the cryogenic-era data, other archival data, and the Chandra $\mathrm{X}$-ray data.

\subsection{Target Selection}

In this section, we first discuss our criteria for picking targets and then review basic properties of each cluster.

\subsubsection{Overview of Target Selection}

The Orion SFR has been the subject of variability studies more than any other SFR. Because Orion is so well-studied, particularly for optical and NIR variability, we elected to include it as a very significant part of our mid-infrared observing effort.

Besides Orion, we selected the cores of 11 additional young clusters for monitoring; see Table 1. Our smaller-field, more embedded cluster sample ("smaller field clusters") was selected based on detailed examination of all the SFRs surveyed with Spitzer by mid-2007, when YSOVAR targets were selected. We chose regions that satisfy the following criteria. (1) A relatively high fraction of Class I sources (for a brief definition of spectral energy distribution (SED) classes, such as Class I, see Appendix B), such that we would obtain monitoring for some of the most heavily embedded objects; (2) a high density of YSOs within one or a few IRAC fields of view (FOVs) typically > 40 YSOs per field; (3) moderate cirrus backgrounds; and (4) minimal problems with crowding or very bright nearby sources. Several of the regions we monitored are very difficult to observe from the ground due to their high level of obscuration. We included IC 1396A even though it is not as embedded because there has already been a significant investment of Spitzer time in monitoring this region (more details on this follow below). NGC 2264, like Orion, has been intensively surveyed for variability from the ground (see, e.g., Makidon et al. 2004; Lamm et al. 2005; Cieza \& Baliber 2007) and from space (see, e.g., Alencar et al. 2010 for CoRoT; Zwintz et al. 2009 for MOST). We focused on the most embedded region of NGC 2264 for the "YSOVAR-classic" part of the program and monitored a much larger region for CSI 2264.

\subsubsection{Cluster Properties}

We now briefly discuss general properties of each of these clusters, in R.A. order (see Tables 1 and 2). We tabulate several characteristics of these clusters in Table 1, including some values from Gutermuth et al. (2009, 2010, hereafter G09). All of these clusters are thought to be between 1 and 5 Myr old. Ages more accurate than that for clusters as young and embedded as these are difficult to obtain, even in a relative sense. Cryogenic Spitzer observations for many of these clusters were discussed in G09, who calculated the Class II to Class I ratio for various regions and sub-clusters within the cryo Spitzer maps. This ratio is easier to obtain than an age and helps place the clusters' evolutionary states into context with each other; it is provided here (in Table 1 and the text below) in part as a link back to existing literature. In general, our observations enclose only the most embedded parts of these clusters; the full cluster membership generally includes objects beyond the regions we monitored. (Orion is different in that the map is much larger than the other smallerfield cluster maps, but, even then, the map does not include all objects thought to be part of Orion.) Thus, we have recalculated the Class II to Class I ratio for only objects with YSOVAR light curves in the same way as was done in G09 (e.g., only for the IR-selected members); this value appears in Table 1, again to place our observations into context with the prior literature. We discuss this parameterization in more detail below in Section 4.1, and consider a different parameterization of the clusters' relative evolutionary states, the ratio of the number of Class I sources to total YSOs; anticipating this discussion, these values are included in Table 1. The total cluster membership we use for this metric includes objects with light curves that we selected using both the IR data from Spitzer and X-ray data from the Chandra X-ray Observatory (Section 3). The X-ray data are discussed further in Section 2.10.

Table 1 also includes an approximate Galactic latitude as a very rough guide to the overall Galactic background/foreground source density expected near each location. Targets close to the Galactic plane (e.g., Serpens Main) have a higher surface density of objects in our FOVs than targets further from the plane.

$A F G L 490$. The cluster associated with AFGL 490 is a portion of the larger Cam OB1 Association, centered on a massive object (8-10 $M_{\odot}$; sometimes the name AFGL 490 is used to refer only to this massive protostar). The cluster is thought to lie between $\sim 900$ pc (Testi et al. 1998) and 1010 pc (Straižys \& Laugalys 2008). We adopt a distance of 900 pc (as do G09). G09, based on the cryogenic Spitzer data for this region, found at least 100 young stars or candidates in this vicinity, and found the overall Class II to Class I ratio to be about 3. Masiunas et al. (2012) also report on the cryogenic Spitzer observations and, incorporating additional data, find many new YSO candidates and a Class II to Class I ratio overall of $\sim 5$. Among the objects with YSOVAR light curves, using the G09 color cuts to identify Class I and II sources (see Section 2.7 and Appendix B), the ratio is $\sim 4.5$. This region is the only cluster in our set of clusters that does not have archival Chandra X-ray observations (see Section 2.10).

NGC 1333. NGC 1333 is on the western edge of the Perseus molecular cloud, and, at only $\sim 235$ pc (Hirota et al. 2008, 2011), it is one of the youngest and most well-studied SFRs, with $>200$ refereed publications. The region is riddled with outflows from young stars (e.g., Plunkett et al. 2013), which can be seen clearly in the Spitzer $4.5 \mu \mathrm{m}$ image of this region. Gutermuth et al. (2008b, 2009) analyzed the cryogenic Spitzer data for this region, and found more than 130 young stars or candidates; the Class II to Class I ratio they found in the core is $\sim 2.7$. For the region where we have light curves, the ratio is $\sim 4.7$. Despite the large number of previous studies in the literature, NGC 1333 has few prior studies specifically investigating time variability. The YSOVAR data will be discussed in detail in L. Rebull et al. (in preparation); Raga et al. (2013) report on proper motions of the outflows in this region using the YSOVAR data.

Orion. Orion has been the subject of extensive variability studies. Haro (1969), Herbig \& Kameswara Rao (1972), and Walker (1978) all conducted pioneering studies of the variability of young stars in Orion, leading to the modern era using 
two-dimensional imaging cameras as initiated by Herbst and his team (Attridge \& Herbst 1992; Choi \& Herbst 1996). They obtained multi-year, optical time series photometry of several regions of the $\mathrm{ONC}$, eventually obtaining light curves for hundreds of YSOs, often spanning several years. Many other groups subsequently obtained time series photometry of other portions of the ONC, using optical (Stassun et al. 1999, 2006, 2007; Rebull 2001; Herbst et al. 2002; Irwin et al. 2007; Rodríguez-Ledesma et al. 2009) and near-IR (CHS01) imaging data. More recently, some far-IR monitoring has been conducted in Orion as well (Billot et al. 2012). In many cases, the light curve shapes are well-fitted by models of rotational modulation via hot or cold spots, normally at moderately high latitudes since the light curves are seldom "flat-bottomed." However, for a significant fraction of the light curves, particularly those in the near-IR, spots do not seem to provide a good explanation for the observed variability (CHS01). We included the ONC as a YSOVAR target because of the substantial amount of extant monitoring available in the literature. Note that it is likely slightly older than most of the other embedded regions studied here. For the cryogenic-era data (see Section 2.7), we used the data reduction, YSO identification, and YSO classification from Megeath et al. (2012), which are very similar to that from G09. Therefore, while there is no ratio of Class II to Class I objects from G09 to report, we calculated this ratio using the Megeath et al. (2012) classifications for only those objects for which we have light curves, obtaining $\sim 9.7$. While this ratio is affected by the much larger region monitored by YSOVAR (only the cores are monitored in most of the other regions), this value is consistent with Orion being slightly older than our other more embedded targets. We adopt a distance of $414 \mathrm{pc}$ from Menten et al. (2007).

Mon R2. Mon R2 appears in G09, with a Class II to Class I ratio in the central region of $\sim 4.7$. This region, part of the Monoceros R2 molecular cloud, is near vdB 67 and 69 (see, e.g., Carpenter \& Hodapp 2008). It is typically thought to be at 830 pc (Herbst \& Racine 1976; Carpenter \& Hodapp 2008). There are several sources that are very bright in the infrared in this location, which affect the completeness of the catalog extracted from the Spitzer data. The Class II to Class I ratio in the region with YSOVAR monitoring is $\sim 6$. These data will be discussed in more detail by L. A. Hillenbrand et al. (in preparation).

GGD 12-15. GGD $12-15$ is a dense core also located in the Monoceros R2 molecular cloud (see, e.g., Carpenter \& Hodapp 2008). As such, we assume it to also be at $\sim 830 \mathrm{pc}$. G09 find a Class II to Class I ratio of $\sim 4.2$; we calculate $\sim 5.8$ for the region with YSOVAR light curves. Several time-variable radio sources are located here (Carpenter \& Hodapp 2008, and references therein). We also monitored this region with Chandra during portions of our YSOVAR Spitzer campaign. These data will be discussed in depth by $\mathrm{S}$. Wolk et al. (in preparation).

NGC 2264. NGC 2264 is thought to be comparable in age to or slightly older than Orion (see, e.g., Ramirez et al. 2004a). This region does not appear in G09 so we do not have a similarly obtained Class II to Class I ratio for comparison. However, the region we monitored as part of the YSOVAR-classic data (original YSOVAR program 61027; see Section 2.2 and Table 2), which is the region discussed here, is centered on the Spokes Cluster (Teixeira et al. 2006), the most embedded portion of NGC 2264 (analogous to the BN region in Orion). We calculate a Class II to Class I ratio in this monitored region of $\sim 3.0$, which is comparable to the ratio for many of the other embedded clusters here. We work only with the YSOVAR-classic data in the present paper; Cody et al. (2014) discuss the much larger CSI 2264 data set. We have adopted a distance to this cluster of 760 pc (Park et al. 2000).

L1688. Lynds 1688 (L1688) is located within the $\rho$ Ophiuchi molecular cloud. It is also one of the best-studied SFRs in this YSOVAR data set, with more than 500 refereed articles. The distance to this region is a subject of some debate. There is recent evidence for $130 \mathrm{pc}$ (Wilking et al. 2008, and references therein), $131 \mathrm{pc}$ (Mamajek 2008), and $120 \mathrm{pc}$ (Loinard et al. 2008, 2013; Lombardi et al. 2008). Lombardi et al. (2008) offer a plausible explanation for the "discrepancy" in the very long baseline interferometry (VLBI) results noted by Wilking et al. (2008): there may be other subregions in Oph at distinct distances, but the evidence is unclear at this point. Our results are not particularly dependent on distance; we have adopted $120 \mathrm{pc}$ as one of the most recent determinations. Lynds 1688 has a high surface density of embedded objects. This region appears in G09 with a Class II to Class I ratio of $\sim 3.0$; we calculate a value of $\sim 1.9$ for the region we monitored. Barsony et al. (2005) first reported YSO variability in the MIR in the objects in this core. By comparison to Infrared Space Observatory data, they found significant variability in 18 out of 85 objects detected, on timescales of years. They found such variability in all SED classes with optically thick disks, and suggest that this might be due to time-variable accretion. The Multiband Imaging Photometer for Spitzer (MIPS; Rieke et al. 2004) data for this region were presented in Padgett et al. (2008); no variability in sources at $24 \mu \mathrm{m}$ was found to a level of $10 \%$ on timescales of hours. Alves de Oliveira \& Casali (2008) recently reported on deep NIR monitoring of this region, with 14 epochs over two years, finding that $41 \%$ of the known YSOs are variable. The $\rho$ Oph core region was included in one of the "calibration" 2MASS fields, and as such has monitoring data in the NIR (Parks et al. 2014) with a cadence of $\sim 1$ day over 3 observing seasons spanning $\sim 2.5$ yr. Parks et al. (2014) found 101 variables, 72 of which are identified with known YSOs in the region. Plavchan et al. (2013) report on YLW 16A, finding a 93 day periodicity. The YSOVAR data are discussed in detail by Günther et al. (2014).

Serpens Main. The Serpens core has been studied for decades, but has become known as "Serpens Main" to distinguish it from the relatively recently discovered embedded star-forming core known as Serpens South (Gutermuth et al. 2008b; see below). The original Spitzer cryo-era data for Serpens Main were presented in Harvey et al. (2007); they found no clear evidence at the $\sim 25 \%$ level for IRAC-band variability in any sources in the field over the $\sim 6 \mathrm{hr}$ timescale of their observations. The Spitzer cryogenic data were also used in G09, who determined the ratio of Class II to Class I objects at $\sim 1.4$, the lowest of all of the clusters from YSOVAR that also appear in G09. It has a high surface density of embedded objects and it is another very well-studied SFR, with more than 500 refereed articles. While the Straižys et al. (1996) distance of 260 pc to Serpens Main is well-cited in the literature, more recent studies (Dzib et al. 2010; Loinard et al. 2013; see also Eiroa et al. 2008) using, e.g., VLBI instead suggest that the distance of $260 \mathrm{pc}$ may be portions of clouds associated with Aquila, and that a better distance for Serpens itself is actually $415 \mathrm{pc}$, which we adopt here. Hodapp (1999) reports on NIR variability of knots, jets, and young stars; in terms of point sources, Hodapp (1999) primarily discusses one particular source (OO Ser) in this region. There was a brief Spitzer monitoring program of 
this region conducted during Cycle 3 as part of guaranteed time (see Table 2); the observations conducted as part of YSOVAR were designed to be well-matched to these observations. We obtain a Class II to Class I ratio of $\sim 2.2$ for the region with light curves.

Serpens South. Serpens South was discovered by Gutermuth et al. (2008b) as a dense, embedded cluster in the SerpensAquila Rift. It is thought that this cluster is at about the same distance as Serpens Main, which we have taken to be 415 pc. From the numbers in Gutermuth et al. (2008b), it has a Class II to Class I ratio of only $\sim 0.7$. Considering only the region we monitored, the ratio is comparable at $\sim 0.9$.

IRAS 20050+2720. Observations from Spitzer and Chandra of the cluster associated with IRAS 20050+2720 (abbreviated IRAS 20050) have been discussed by Günther et al. (2012). This cluster is part of the Cygnus Rift and is likely at $\sim 700$ pc (Günther et al. 2012, and references therein). G09 determined the ratio of Class II to Class I objects to be $\sim 1.9$; in the region with light curves, we obtain $\sim 2.2$. The YSOVAR data for this cluster will be discussed in detail by K. Poppenhaeger et al. (in preparation).

IC 1396A. IC 1396A is the most prominent globule in the IC 1396 complex; this cluster is sometimes called the Elephant Trunk Nebula. Morales-Calderón et al. (2009) presented the first Spitzer monitoring of young stars, centered on this target. This region is also likely to be very young, but it is not particularly embedded, at least compared to other YSOVAR clusters. It is not included in G09, but over the entire region within which light curves were obtained, we obtain a Class II to Class I ratio of $\sim 11.6$. Because IC $1396 \mathrm{~A}$ is at a high ecliptic latitude (see Section 2.3 below for discussion of ecliptic latitude dependencies), there are light curves with only a few single-band points for many objects. For the much smaller number of objects that have light curves in both IRAC-1 and -2, we obtain a much lower Class II to Class I ratio of $\sim 7.9$, though within Poisson errors calculated assuming independent errors in the numerator and denominator, these values are consistent $(11.6 \pm 4.0$ and $7.9 \pm 2.8)$. We assume it is at a distance of $\sim 900 \mathrm{pc}$ (Contreras et al. 2002).

Ceph $C$. Ceph $\mathrm{C}$ is part of the Cep OB 3 molecular cloud, and is included in the G09 study, with a Class II to Class I ratio of $\sim 2.3$ obtained in that paper. G09 used a distance of 730 pc (Blauw 1964); we adopt a distance of 700 pc based on maser parallax from Moscadelli et al. (2009). Ceph C is one of the less well-studied clusters in our set. For the region with any light curves (see Section 2.3 below), we obtain a Class II to Class I ratio of $\sim 3.8$. Because it, like IC 1396A, is at a high ecliptic latitude, we repeated this calculation for the much smaller number of objects that have light curves in both IRAC1 and -2 , obtaining a ratio of $\sim 3.2$; again assuming Poisson counting statistics, these ratios are comparable $(3.8 \pm 1.1$ and $3.2 \pm 1.0$ ). This cluster was monitored during our YSOVAR campaign with Chandra as well; these data will be discussed in depth by K. Covey et al. (in preparation).

\subsection{Warm Spitzer Observations: General Properties}

To better manage the observation planning and data downloading for the 12 clusters we observed, we separated each cluster into an individual observing program. The individual observing programs and clusters are listed in Table 2; one can download the data from the Spitzer Heritage Archive using these program numbers. We will deliver all of our extracted photometry to the Spitzer Science Center (SSC) and Infrared Science
Archive (IRSA ${ }^{29}$ ) for public access via IRSA tools, and through those tools, the Virtual Observatory.

Most of the observations were IRAC mapping mode Astronomical Observation Requests (AORs) using full-array $12 \mathrm{~s}$ high-dynamic-range (HDR) mode (which is defined such that a 0.4 and $10.4 \mathrm{~s}$ exposure is obtained at each pointing-see the IRAC Instrument Handbook, available at the SSC/IRSA Web site $^{30}$ ). In some cases, as noted in Table 2, the AORs were staring AORs, meaning that they were continuous or semi-continuous observations without dithering or mapping. These staring data will be discussed in other YSOVAR papers. The present paper is limited to the HDR mapping observations (and is largely further restricted to the "fast cadence" observations; see Section 2.4).

Roughly half of the original YSOVAR time allocation was devoted to observations of Orion. The Orion Spitzer campaign included a $\sim 0.9 \mathrm{deg}^{2}$ region centered on the Trapezium cluster in Orion; this is far larger than can be obtained in a single AOR at a single epoch. The observed area was thus broken into five segments with a central region of $\sim 20^{\prime} \times 25^{\prime}$ and four flanking fields. The central part was observed in full array mode with $1.2 \mathrm{~s}$ of exposure time and 20 dither positions to avoid saturation by the bright nebulosity around the Trapezium stars. The remaining four segments of the map were observed in $12 \mathrm{~s} \mathrm{HDR}$ mode, and four dither positions. More details on these original YSOVAR Orion observations appear in MC11. We reallocated time within the YSOVAR time budget to follow up on some eclipsing binaries in Orion (Morales-Calderón et al. 2012). We also obtained time in Cycle 7 to follow up on some AA Tau analogues in Orion presented in MC11. These follow-up observations did not map the entire Orion region.

The rest of the original YSOVAR time allocation was divided among the rest of the SFRs. In contrast to Orion, for the 11 other clusters, the monitoring observations are one or at most a few IRAC FOVs; see the last column of Table 2. As noted above, we sometimes refer to these 11 other clusters as "the smaller field clusters."

The observations are typically spread over weeks to months, usually at a cadence of about twice per day, but with an interval between observations that varied both by design (to reduce aliasing problems) and due to constraints imposed by other Spitzer programs or infrastructure operations that were executed during the same campaigns (further details on the cadence follow below in Section 2.4).

NGC 2264 was included as a smaller-field cluster to be monitored as part of the original YSOVAR program, and while this original program was still executing, CSI 2264 was approved. We continued to execute the original YSOVAR smallfield observations in this region (program 61027 in Table 2), and these small-field regions are discussed here, since they resemble the rest of the original YSOVAR programs more closely than they resemble CSI 2264.

Two of our clusters, IC 1396A and Serpens Main, were monitored in the cryogenic era with Spitzer with the primary intention of monitoring changes in these objects in the Spitzer bands (as opposed to removing artifacts). In YSOVAR, we reobserved these clusters in the same way so that the same objects continue to be monitored, and we re-reduced all of these data in the same fashion as discussed here. In the context of this paper, we are not particularly focused on these cryogenic-era

\footnotetext{
29 http://irsa.ipac.caltech.edu/

30 http://irsa.ipac.caltech.edu/data/SPITZER/docs/
} 


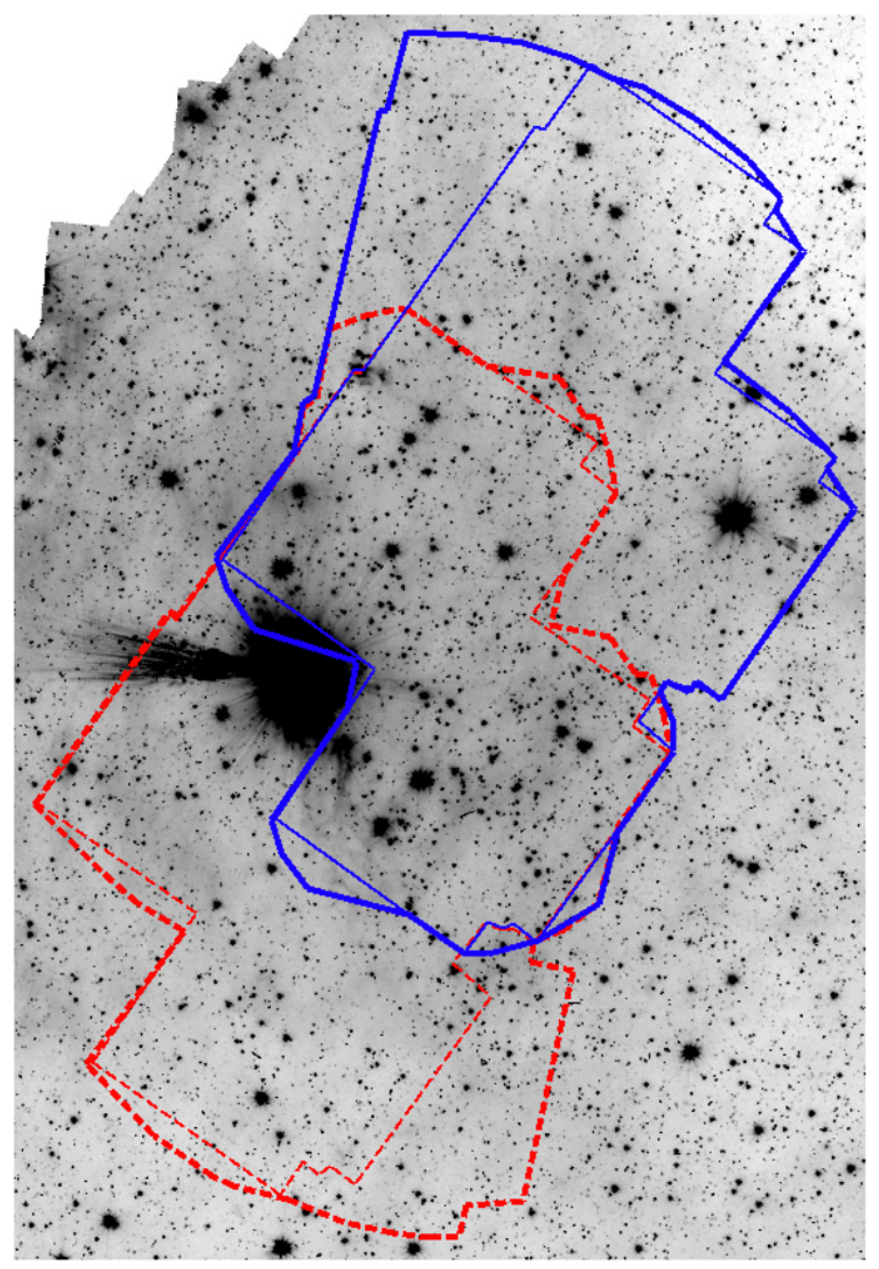

Figure 1. Approximate sky coverage for a summed-up image consisting of all epochs of YSOVAR AFGL 490 observations, superimposed on a reverse grayscale image of AFGL 490 at $4.5 \mu \mathrm{m}$ obtained during the cryogenic mission. The thicker solid blue line is $3.6 \mu \mathrm{m}$ and the thicker red dashed line is $4.5 \mu \mathrm{m}$. A single epoch of observation is also indicated by thinner solid blue and dashed red lines, with the difference between the single epoch and the larger polygon due to (ecliptic-latitude-dependent) field rotation effects. North is up and east is to the left. The distance between the farthest north and farthest south coverage here is $\sim 27^{\prime}$. The field rotation here is not as significant as in those with ecliptic latitudes $\sim 60^{\circ}$. There is no Chandra coverage for this cluster. Similar figures for the remaining 11 clusters are included in Appendix C.

(A color version of this figure is available in the online journal.)

monitoring data; however, these data will be included in the YSOVAR cluster-specific papers.

\subsection{Warm Spitzer Observations: Footprints and Operational Constraints}

Because of the nature of Spitzer and IRAC, the footprints of our observations and how they vary with time are both complicated issues. We now discuss these issues as they pertain to YSOVAR observations.

Figures 1-13 present the outline (footprint) of the YSOVAR observations. The original YSOVAR Orion observations (Figure 3) and the CSI 2264 observations (Figure 7) cover a substantially larger area than those for the other smaller-field clusters. Chandra footprints are included in these figures for reference and discussed below in Section 2.10.

The focal plane of the IRAC camera is such that the 3.6 and $4.5 \mu \mathrm{m}$ FOVs are not the same; data are obtained in both FOVs at once, with one field placed on the target of interest, and the

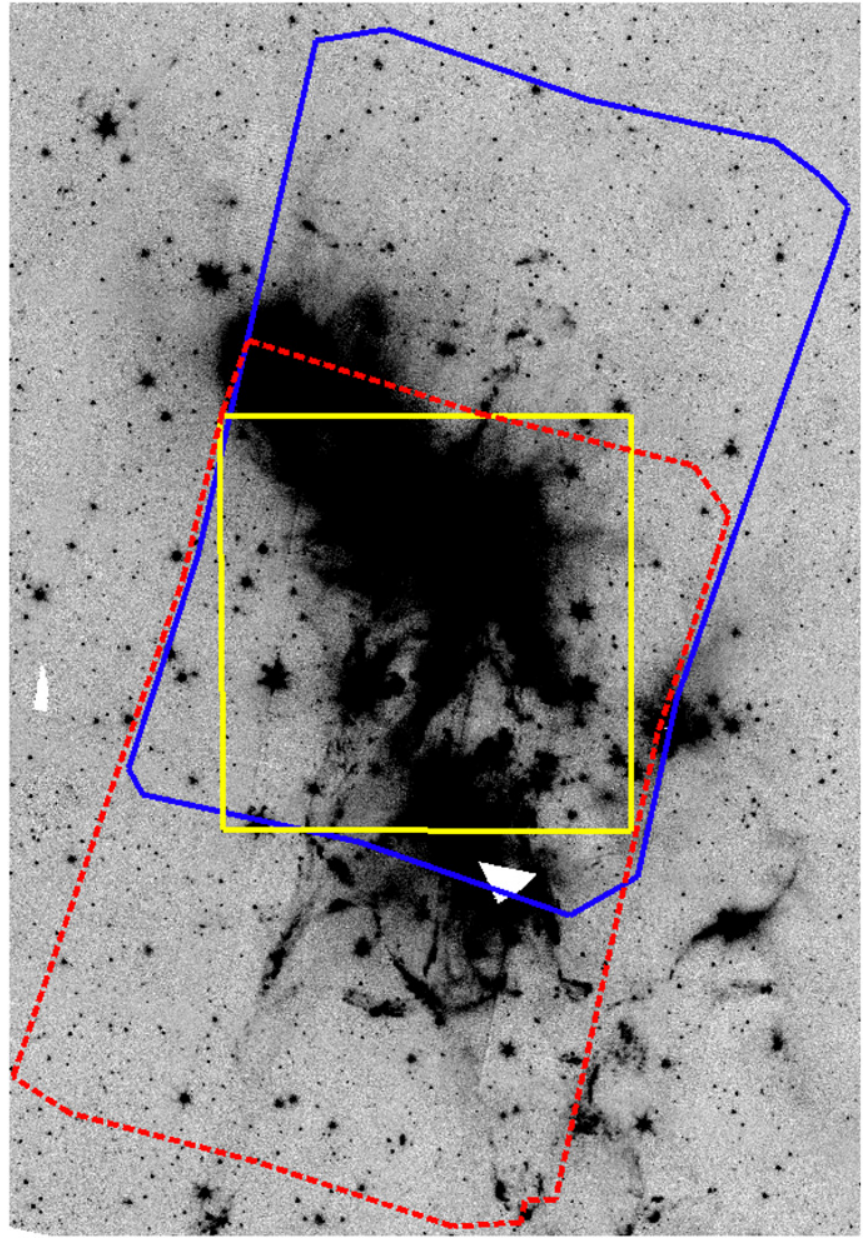

Figure 2. Same as Figure 1, but for our NGC 1333 observations, with the north-south boundary extremes separated by $\sim 24^{\prime}$. The field rotation here is essentially zero. The yellow polygon indicates the approximate region covered by Chandra observations.

(A color version of this figure is available in the online journal.)

other obtaining serendipitous data in a non-overlapping $\sim 5^{\prime} \times 5^{\prime}$ field with a center offset $\sim 6.5$ from the target field; see the IRAC Instrument Handbook for more details. The placement of these FOVs also changes with time. For all Spitzer observations, as discussed in the Spitzer Space Telescope Handbook (also available at the SSC/IRSA Web site ${ }^{31}$ ), the ecliptic latitude of the target defines when one can observe the target and for how long. At any one time, Spitzer can observe in an annulus defined by the operational pointing zone, which can be conceptually summarized as "neither too close nor too far away from the Sun." It is about $40^{\circ}$ wide, and rotates with the Sun at a rate of about a degree a day. An object near the ecliptic plane can thus only be observed for a period of about 40 days twice a year; objects near the ecliptic pole can be observed at any time during the year for as long as needed. Related to this, the IRAC FOV, as projected onto the sky for any given object on the ecliptic equator, is at an essentially constant angle with respect to north for the duration of the $\sim 40$ day observing window, and, $\sim 6$ months later, is flipped by $180^{\circ}$ but then also essentially constant for that $\sim 40$ day observing window. However, the FOV for an object at the ecliptic pole rotates by about a degree a day.

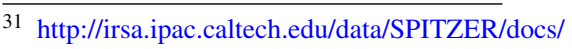




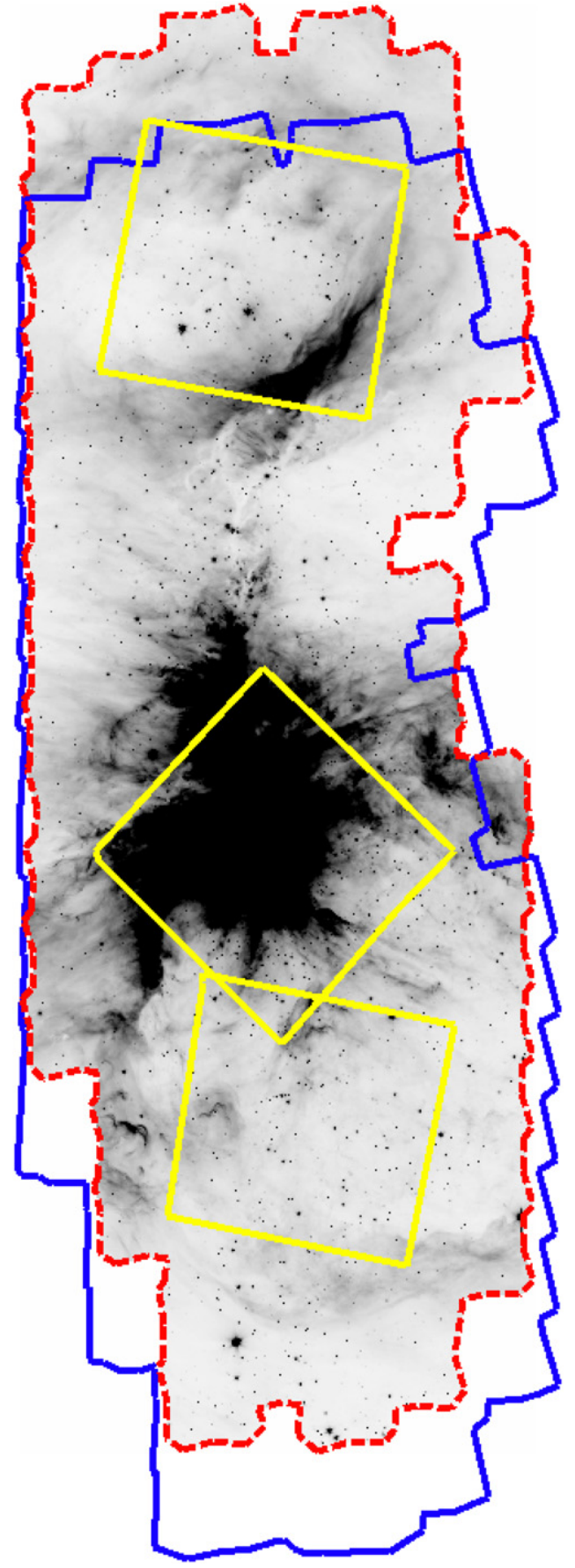

Figure 3. Same as Figure 2, but for our Orion observations, with the north-south boundary extremes separated by $\sim 1$. 6 . The central Chandra (yellow) polygon is the approximate footprint from the deep ONC observation (Getman et al. 2005); the northern and southern Chandra pointings are much shallower, and are taken from Ramirez et al. (2004b). The background image here is an IRAC-2 image from the YSOVAR campaigns (as opposed to a cryo-era image, as it is for most of the other figures like this).

(A color version of this figure is available in the online journal.)

For each of the figures showing the IRAC footprints (Figures 1-13), the projected outline of the observation covered by the entire YSOVAR data set is indicated on top of a $4.5 \mu \mathrm{m}$ observation obtained (in most cases) during the cryogenic era. The different regions observed by the 3.6 and $4.5 \mu \mathrm{m}$ cameras are identified; the nominal target of the observation is covered in both FOVs. In addition to the target of the observations, there are serendipitous data obtained offset from the target area, as seen

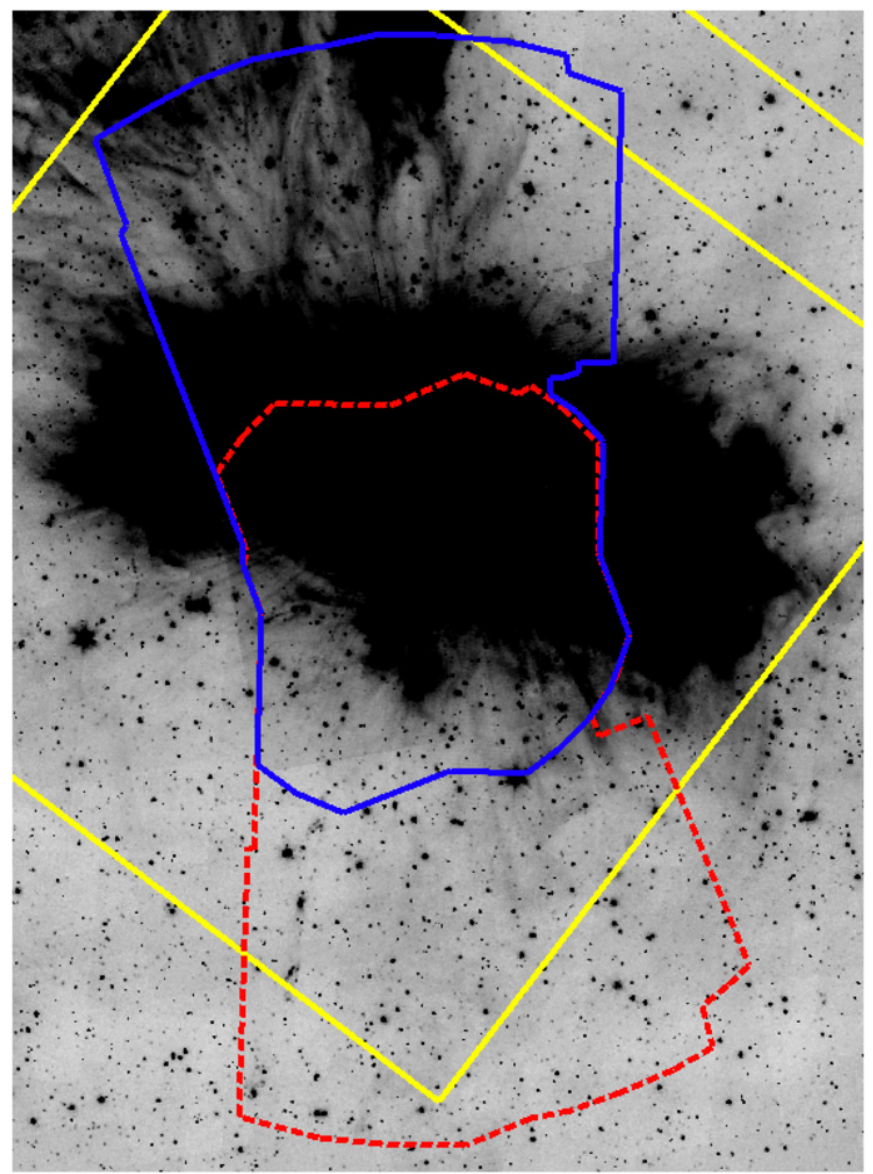

Figure 4. Same as Figure 2, but for our Mon R2 observations, with the north-south boundary extremes separated by $\sim 21^{\prime}$. Field rotation, while present, is not as substantial for this field as it is for others of our clusters. There is a small amount of additional X-ray data (not relevant for this project) to the upper right, and a portion of that footprint can be seen.

(A color version of this figure is available in the online journal.)

in the figures. The footprint of the serendipitously obtained data in each band can change considerably depending on the time of observation and the ecliptic latitude of the target. For targets at higher ecliptic latitudes, where field variation with time is important, a single epoch of observation is indicated in the figure in addition to the area covered by the entire YSOVAR data set.

The approximate ecliptic latitude for each target is included in Table 2 as a rough indication of how long the observing window was and how much field rotation one can expect to see in these figures. For Orion, we designed our observations to be less sensitive to rotation; that plus the fact that the Orion map contains many FOVs means that the rotation is much less of a factor in terms of how much of the sky may be included in both channels (as opposed to only one). For the smallerfield cluster observations containing only one to four IRAC FOVs, a primary target field is monitored in both IRAC bands, but substantial serendipitous monitoring data have also been obtained, typically in just one band. For most of the smallerfield YSOVAR cluster targets, such as NGC 1333 (Figure 2), the central region has complete two-band coverage and most of the objects in the region of the single-band coverage north and south of the target field have data over most, if not all, of the campaign. For L1688 (Figure 8), with an ecliptic latitude of $-3^{\circ}$, the field rotation is essentially zero during one $\sim 40$ day window. However, it was monitored over more than one window, so the observations flip by $180^{\circ}$ for the next $\sim 40$ day window. 


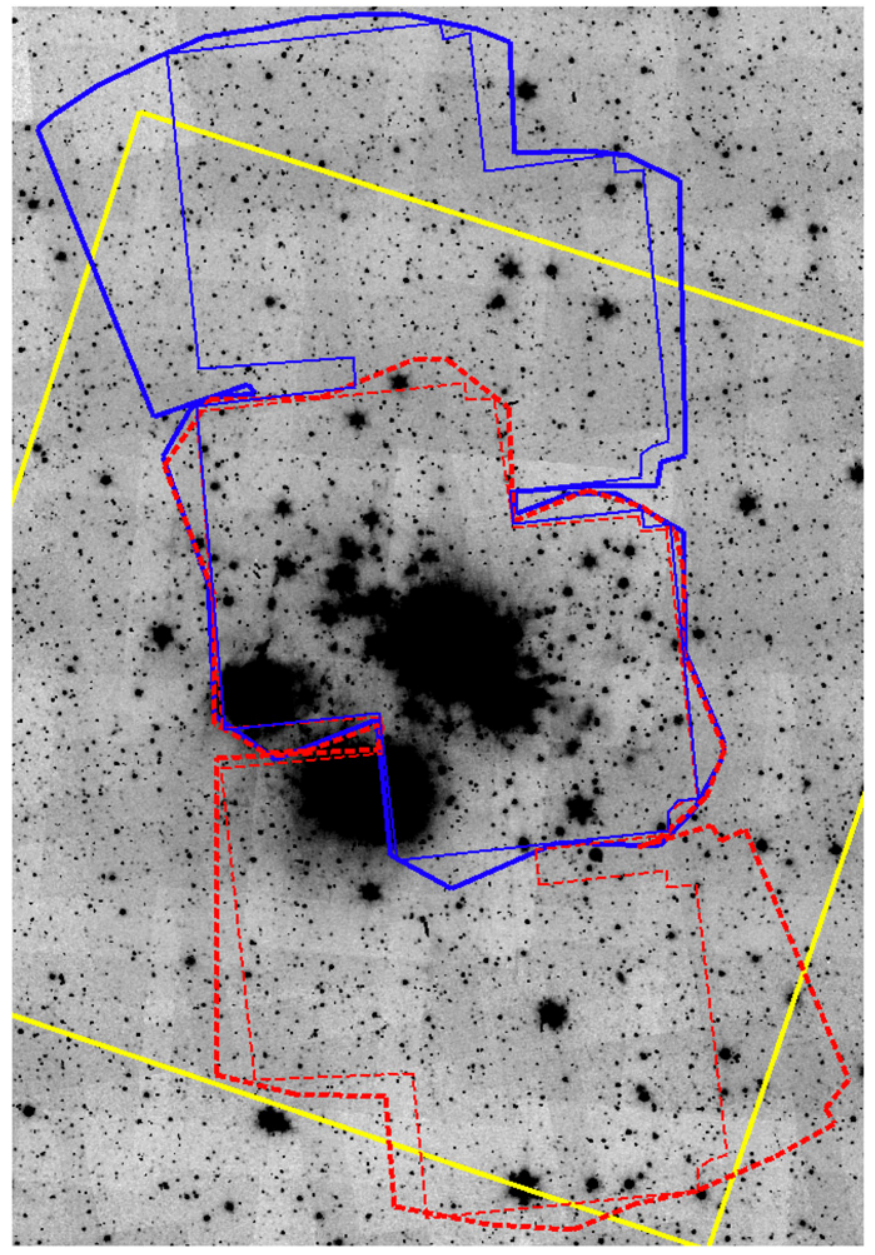

Figure 5. Same as Figure 2, but for our GGD 12-15 observations, with the north-south boundary extremes separated by $\sim 23^{\prime}$. A single epoch of observation is also indicated by the thinner blue solid $(3.6 \mu \mathrm{m})$ and red dashed $(4.5 \mu \mathrm{m})$ lines to give an indication of the magnitude of the field rotation for this field.

(A color version of this figure is available in the online journal.)

(The targets of observation in Figure 8 are the three regions with brightest stars and nebulosity in the centers of the three "stripes" of coverage.) In contrast, for IC 1396A or Ceph C (ecliptic latitudes of $\sim 60^{\circ}$ ), the central $5^{\prime} \times 5^{\prime}$ region is covered throughout the monitoring window, but the rapid field rotation creates a "fan" of coverage such that the serendipitous coverage in the outer periphery of those targeted regions have data in just one band, and only for a fraction of the campaign. Some objects are monitored in $3.6 \mu \mathrm{m}$ at the beginning of the campaign, and in $4.5 \mu \mathrm{m}$ at the end of the campaign (see Figures 12 and 13).

If one considers only objects with light curves in both channels for the smaller-field clusters, even for the fields that do not rotate, one loses a significant fraction of the available data; while the nominal targets of our observations are the regions with two-band coverage, there are still good light curves for cluster members in the regions with coverage in only one band. Generally, for the smaller-field clusters, we did not require data in both channels, and instead retained all light curves for our analysis, even if obtained in only one channel (Section 3.2). Note also that the highest ecliptic latitude among our targets is $\sim 65^{\circ}$; no higher ecliptic latitude targets were included, despite the possibility of much longer observing windows, at least in part

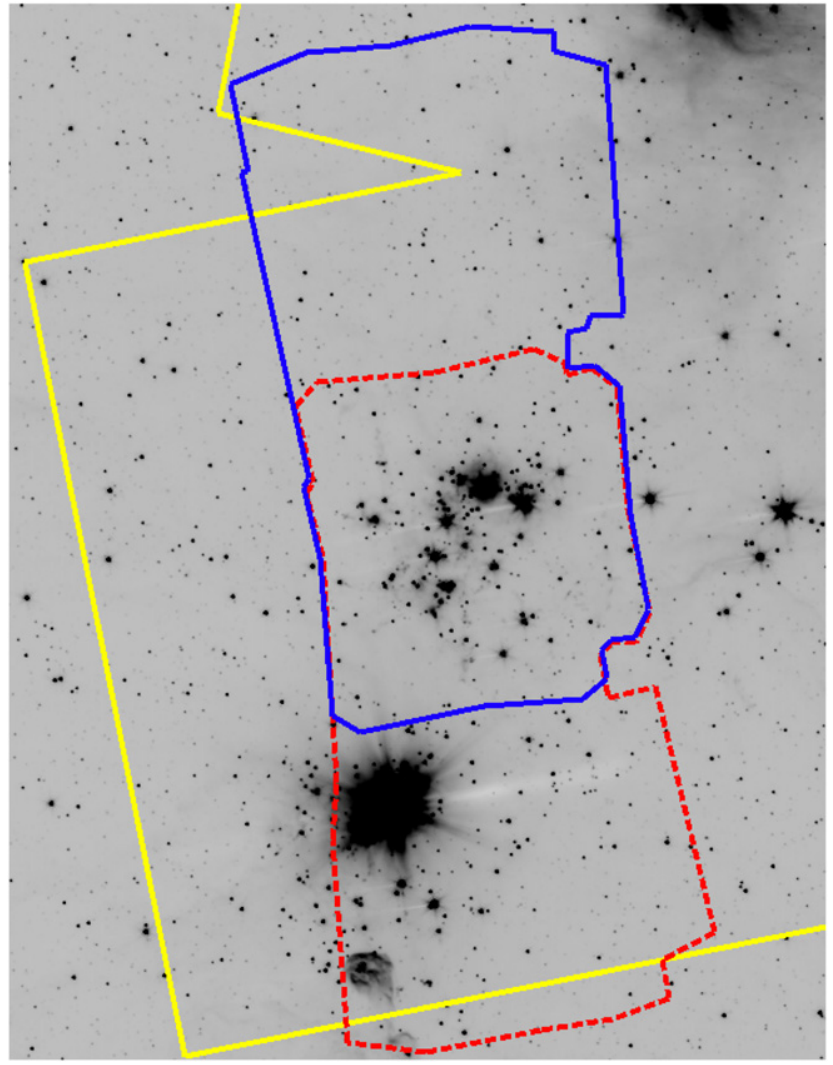

Figure 6. Same as Figure 2, but for our NGC 2264 observations, with the north-south boundary extremes separated by $\sim 20^{\prime}$; the underlying image comes not from the cryogenic era, but from the CSI 2264 observations. Note that this is only the field covered as part of the original YSOVAR observations, e.g., program 61027. For CSI 2264, see the next figure. Field rotation, while present, is not substantial. The background image here is an IRAC-2 image from the CSI 2264 campaign (as opposed to a cryo-era image, as it is for most of the other figures like this).

(A color version of this figure is available in the online journal.)

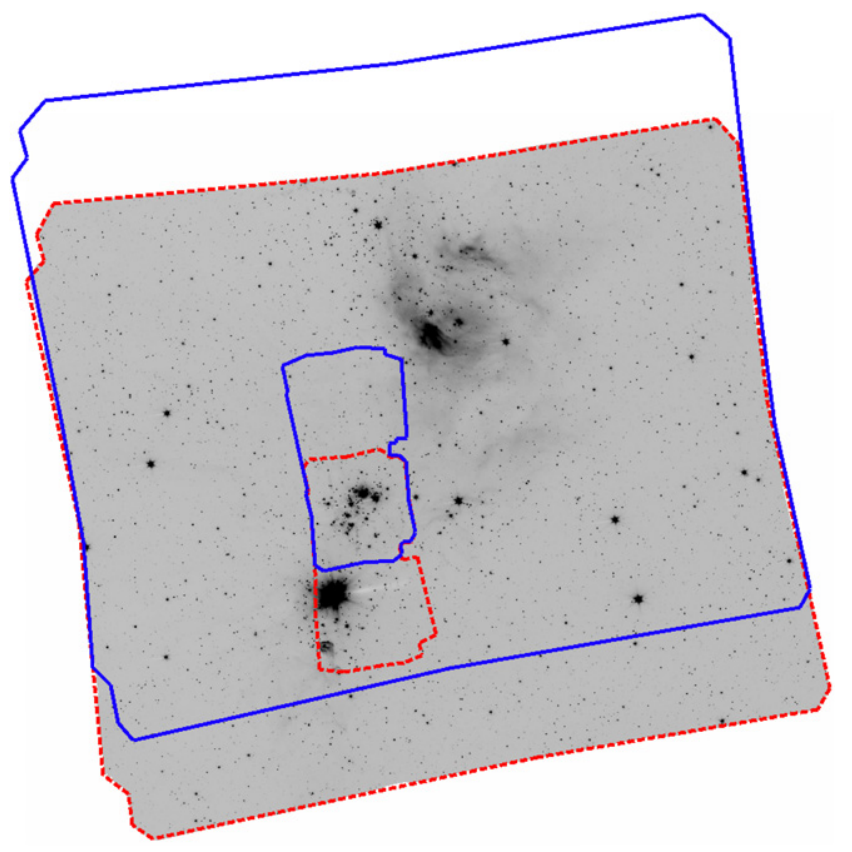

Figure 7. Same as Figure 2, but for our CSI 2264 observations, with the north-south boundary extremes separated by $\sim 0.9$. The underlying image comes from the CSI 2264 observations. The smaller footprints are the YSOVARclassic monitored region from the previous figure.

(A color version of this figure is available in the online journal.) 


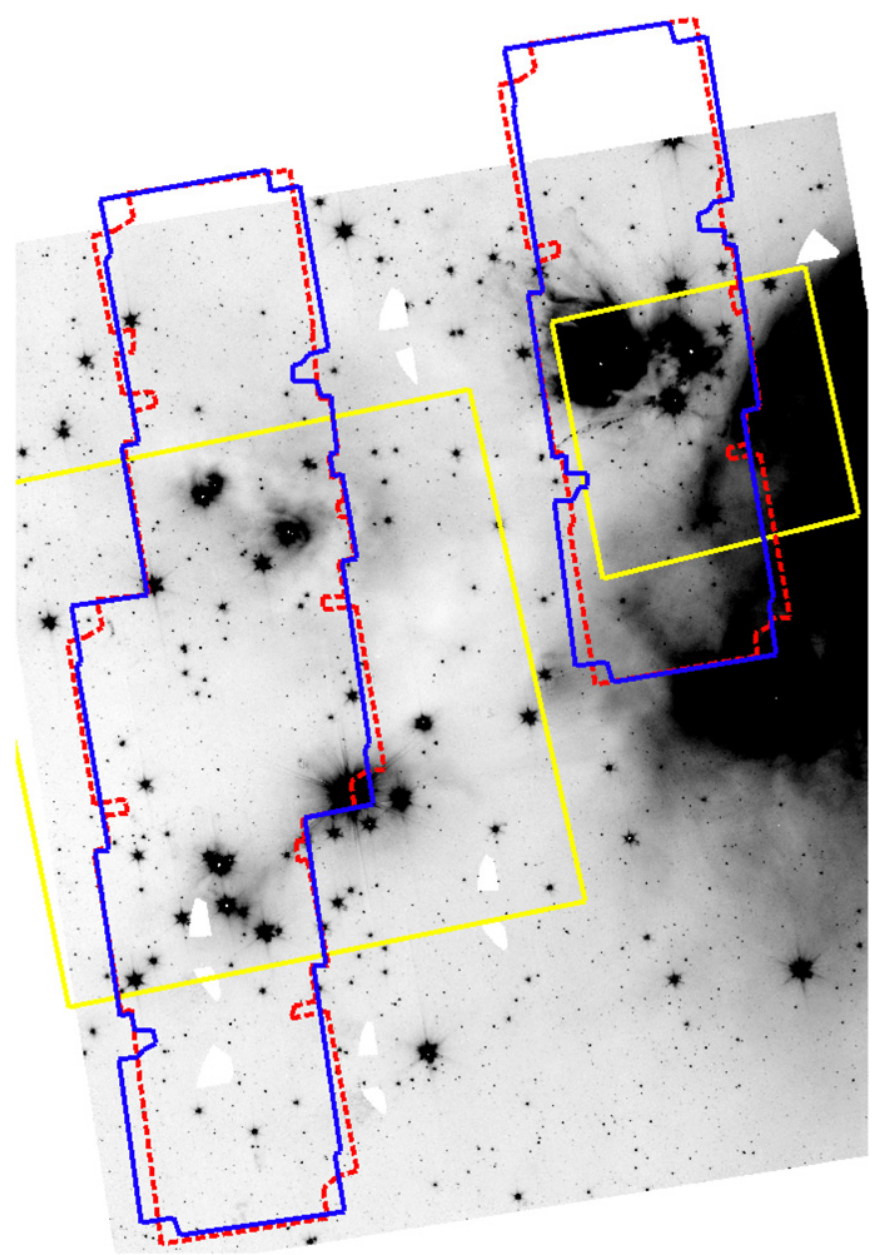

Figure 8. Same as Figure 2, but for our L1688 observations, with the north-south boundary extremes separated by $\sim 38^{\prime}$. The field rotation is essentially nonexistent during one $\sim 40$ day window, and then flips by $180^{\circ}$ for the next $\sim 40$ day window. The targets of observation are the three regions with brightest stars and nebulosity in the centers of the three "stripes" of coverage.

(A color version of this figure is available in the online journal.)

because we could not find any suitable SFRs at these latitudes at the time we planned our observations. The field rotation for these targets at $\sim 65^{\circ}$ is already substantial.

In general, because of the field rotation, as any given object moves into (or out from) the mapped region, the first (or last) few epochs may be unusable as the object may appear in only a fraction of the dithers at that position and the photometry may thus be compromised by edge effects. Substantial field rotation with time can have a significant effect on faint stars near bright stars. Diffraction spikes of bright stars rotate with time within the IRAC FOV, so if a target star falls near a diffraction spike, this can introduce a source of false longer timescale variability; see also Section 2.5.

We note here that the low ecliptic latitude of L1688 (and specifically the $180^{\circ}$ flip between observing windows) enables Günther et al. (2014) to use these observations to characterize possible artifacts in the light curve related to the position in each frame. These tests confirm that the statistical criteria for selecting variable sources (see Section 5 below) is restrictive enough that differences between positions within the frame cannot be a primary contributor for the sources identified as variable.

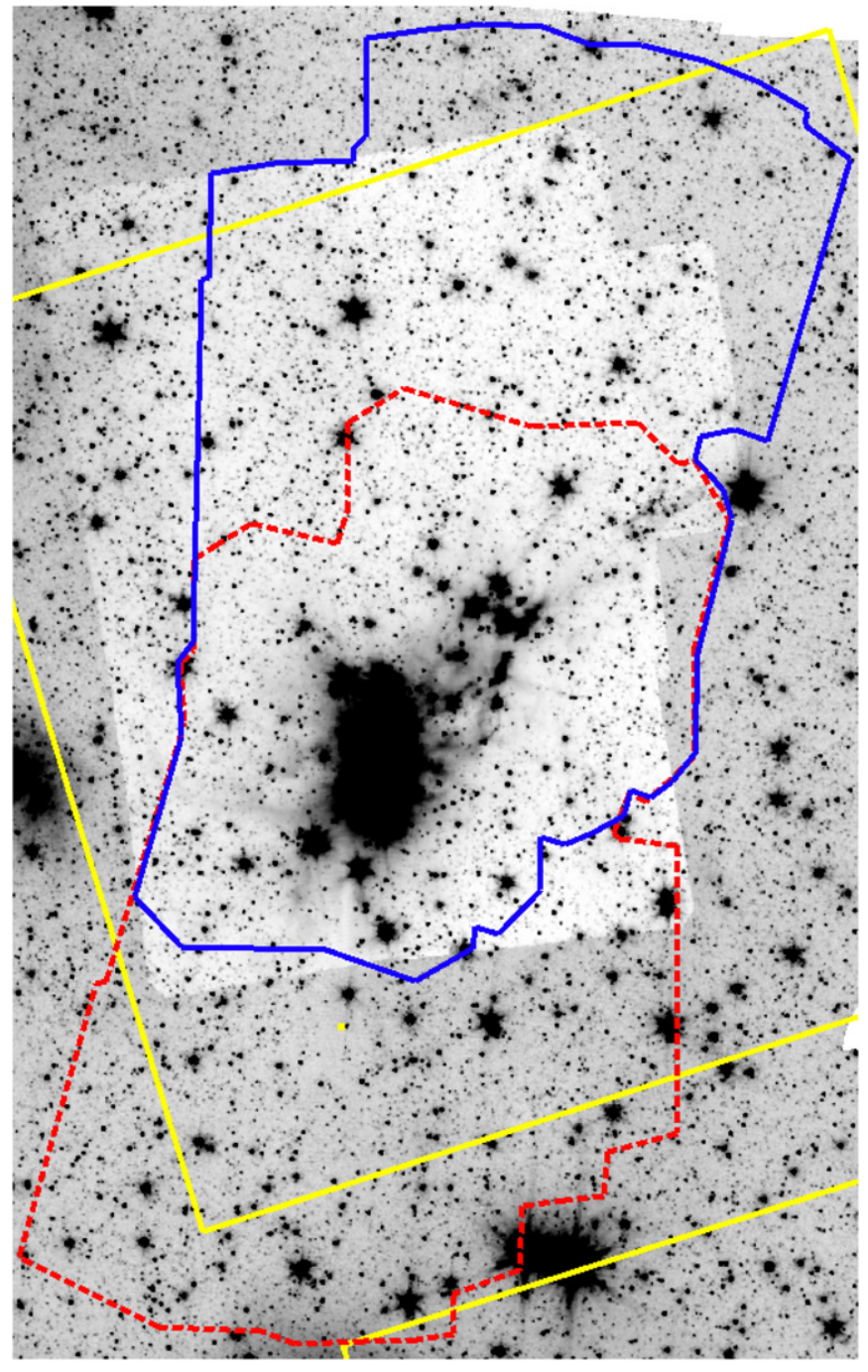

Figure 9. Same as Figure 2, but for our Serpens-Main observations, with the north-south boundary extremes separated by $20,019,990,167 \sim 23^{\prime}$. Field rotation, while present, is not substantial. There is a small amount of additional $\mathrm{X}$-ray data (not relevant for this project) to the lower right, and a portion of that footprint can be seen.

(A color version of this figure is available in the online journal.)

\subsection{Warm Spitzer Observations: Observing Cadence}

More than $95 \%$ (524 hr) of our original YSOVAR Spitzer Cycle 6 program observing time was devoted to a "fast cadence" mode, designed to be sensitive to timescales from $\sim 0.15$ to $\sim 40$ days, consistent with the known timescales of YSOs due to accretion-related flickering, rotational modulation of star spots, and other effects. The upper limit to this range of timescales was set by the typical duration of Spitzer visibility windows near the ecliptic plane, as discussed above in Section 2.3.

Sampling a light curve at evenly spaced intervals introduces period aliasing, and a bias toward the detection of false periods at integer fraction multiples of the sampling interval (see, e.g., Plavchan et al. 2008b or Dawson \& Fabrycky 2010). This period aliasing is common at integer fraction multiples of one day in ground-based photometric surveys due to the natural day-night cycle of the Earth's rotation. Given typical YSO rotation periods of one to several days, we therefore chose to execute our program with a cadence designed to be compatible 


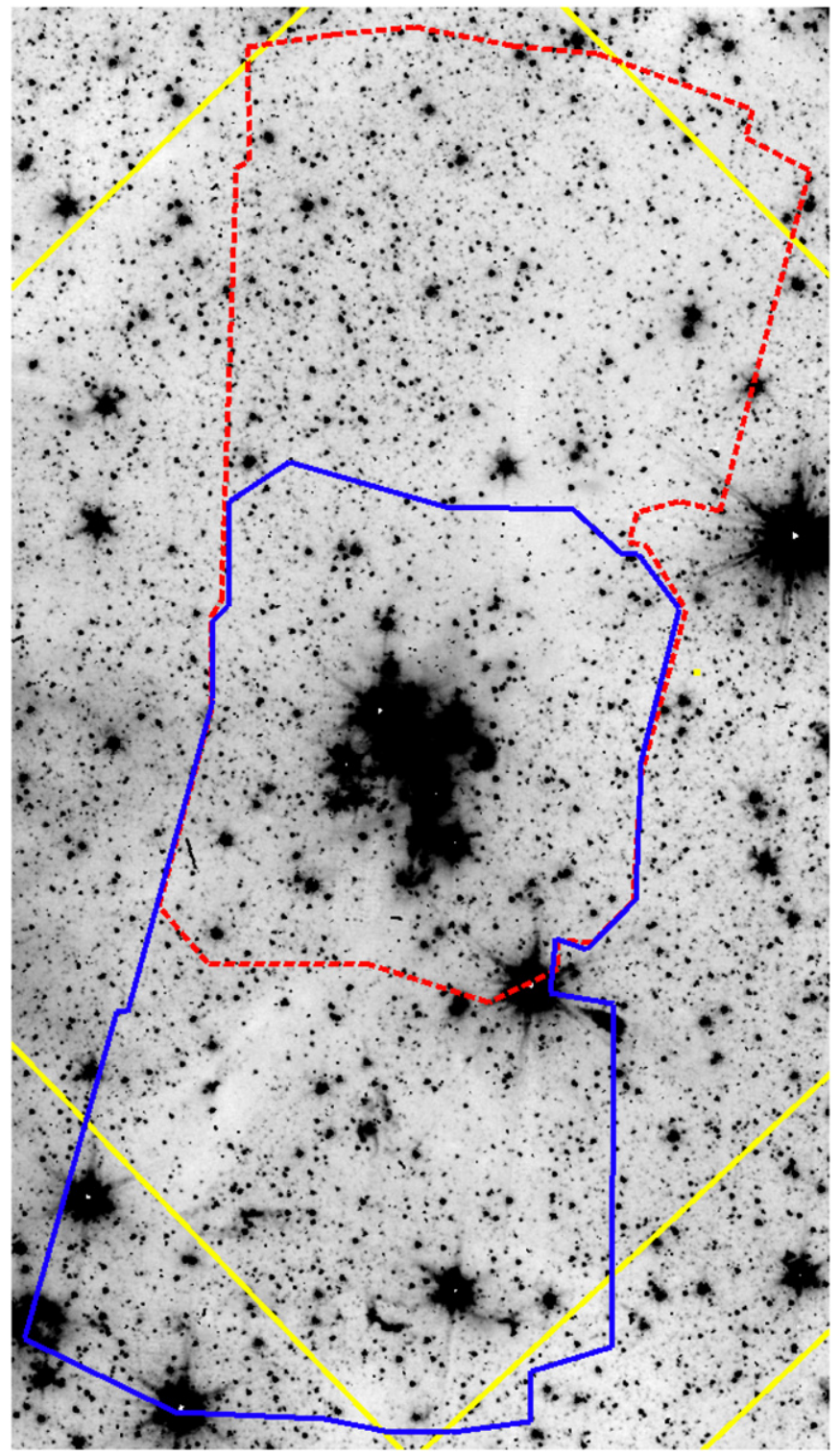

Figure 10. Same as Figure 2, but for our Serpens-South observations, with the north-south boundary extremes separated by $\sim 21^{\prime}$. Field rotation, while present, is not substantial. There is a small amount of additional X-ray data (not relevant for this project) to the lower right, and a portion of that footprint can be seen.

(A color version of this figure is available in the online journal.)

with the Spitzer scheduling, and minimize period-aliasing while retaining sensitivity to variability on a broad range of timescales.

The fast cadence mode for each region had a total time baseline of $\sim 40$ days, varying depending on the actual duration of the visibility window, with additional days on either end of the visibility window for scheduling flexibility. The actual total duration of the fast cadence observations for each cluster is listed in Table 3. In particular, for AFGL 490, Mon R2, GGD 12-15, IRAS 20050+2720, IC 1396A, and Ceph C, we originally planned a 42 day timespan; for Orion, Serpens Main, and Serpens South, we planned a 38.5 day timespan; and for NGC 1333, NGC 2264 and L1688, we planned a 35 day timespan. We divided the time baseline into a repeating set of 3.5 day sub-cadences to ease scheduling. Within each 3.5 day period, we made eight visits, with time steps between visits of $4,6,8,10,12,14$, and $16 \mathrm{hr}$, respectively. By using a linearly increasing time step, we were able to evenly sample in Fourier space a range of higher frequency (shorter timescale) photometric variability than we would have been able to otherwise. Additionally, we were also able to minimize the total amount of necessary observing time to sample these high frequencies and to minimize the period aliasing. For comparison, splitting 8 visits evenly across a 3.5 day cadence would have removed our sensitivity to variability timescales shorter than $\sim 0.5$ day.

To further accommodate scheduling flexibility, we also included a window of $\pm 2 \mathrm{hr}$ in which to obtain a given epoch of observation for our fast cadence observations. This $\pm 2 \mathrm{hr}$ window effectively resulted in a randomization of the cadence about our desired times of observation, which had the additional benefit of further reducing period aliasing, in particular aliases with periods of 3.5 days. We also allowed for interruptions due to data downlink transfers and higher priority observations such as staring mode observations of transiting exoplanets. We are grateful to the SSC scheduling staff for accommodating this fairly complex cadence over the two years of the survey program.

Overall, we obtained $\sim 40-100$ epochs per cluster, which are visualized in Figure 14. Table 2 lists all the clusters, with information about the observations (including the total number of epochs); Table 3 summarizes only the fast cadence observations, including typical values of the minimum and maximum size of the timestep between epochs, and the total time baseline. Our unevenly spaced epochs enable a characterization of different types of observed variability on a variety of timescales; for further discussion, see below (Section 5.4). While periodic analysis tools take advantage of such unevenly spaced data, Findeisen et al. (2014) point out that for auto-correlation functions (ACFs) of non-periodic light curves, timescale sensitivity may be limited by the largest (not the smallest) adjacent timestep in the time series.

For three SFRs (IC 1396A, L1688, and Ceph C) with longer visibility windows, or that we observed in multiple visibility windows, we also observed in a "slow cadence." Besides the 42 day fast cadence, we executed visits with time steps between visits of $1,2,3,4,5$, etc. days to cover the remaining duration of the visibility window. These observations constituted $\sim 5 \%$ of our Cycle 6 observing program $(25 \mathrm{hr})$, and these observations allowed us to be sensitive to variability timescales of $\sim 40$ days to $\sim 2 \mathrm{yr}$, e.g., to objects such as KH15D (e.g., Winn et al. 2006) and WL4 (Plavchan et al. 2008a), as well as long term trends on timescales of a year identified in the NIR (e.g., Parks et al. 2014). For L1688 and Ceph C, we also executed this slow cadence observing mode during the three other visibility windows during our survey. Additionally, Orion had some slower cadence follow up, and NGC 2264 had the CSI 2264 program at a different cadence.

For the primary statistical analysis discussed in this paper, we use the "standard statistical sample," e.g., only the fast cadence data (where there are at least five epochs in the light curve; see Section 3 below). For about half of the clusters, this is the entirety of the data set; see Tables 2 and 3, and the red points in Figure 14. A detailed analysis of the ensemble of all of these observations for each cluster will be presented in papers in preparation, customized to each cluster.

For completeness, we note here the following specifics about those clusters with data beyond the YSOVAR fast cadence. L1688, IC 1396A, and Ceph C all have slow cadence data. Orion has slower cadence data over a second year. Data for 


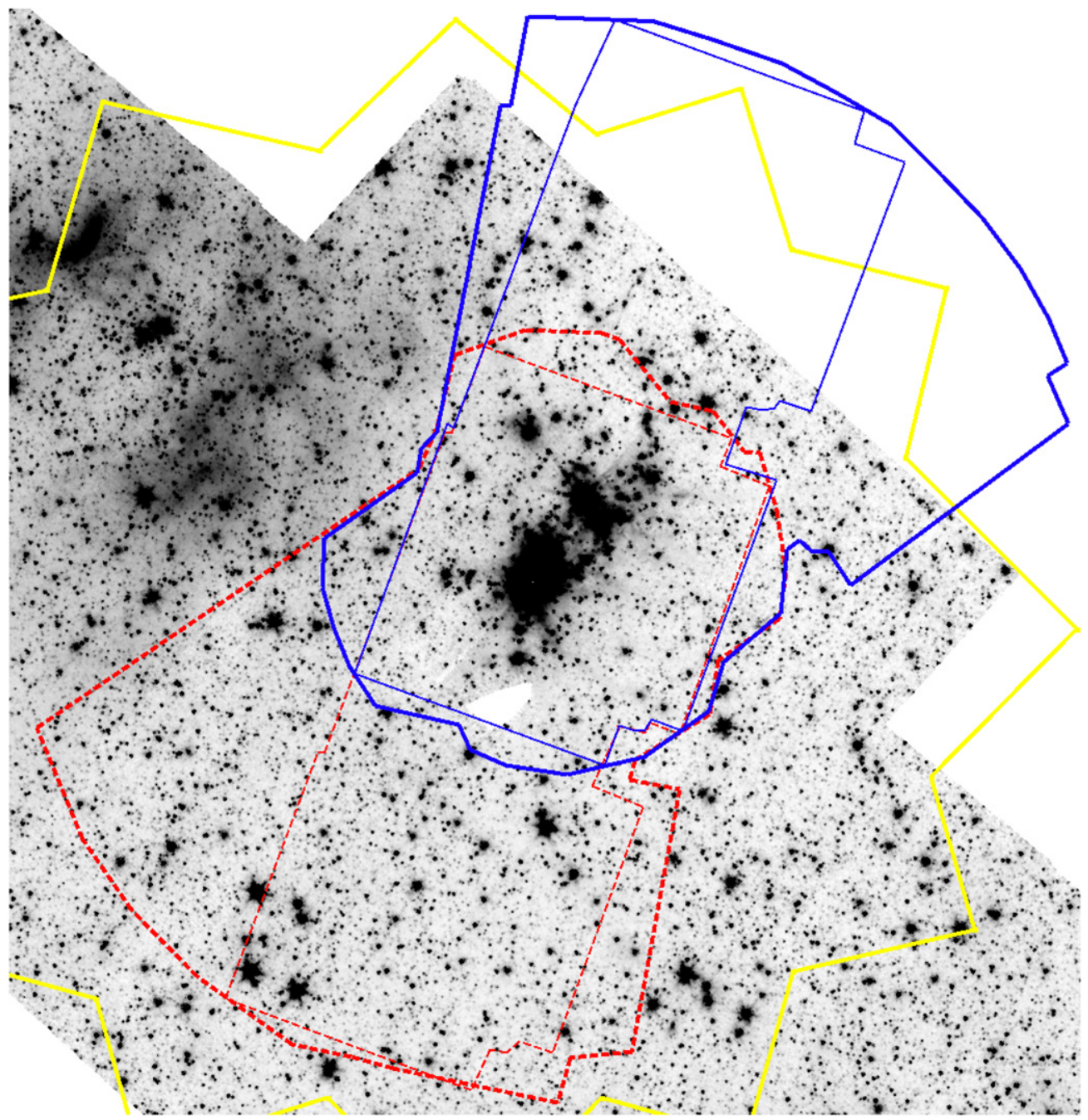

Figure 11. Same as Figure 2, but for our IRAS 20050 observations, with the north-south boundary extremes separated by $\sim 21^{\prime}$. As in Figure 5, a single epoch of observation is also indicated by the thinner blue solid $(3.6 \mu \mathrm{m})$ and red dashed $(4.5 \mu \mathrm{m})$ lines. Multiple visits of Chandra data at multiple roll angles were obtained here, resulting in the star-shaped Chandra coverage.

(A color version of this figure is available in the online journal.)

Table 3

Summary of YSOVAR Fast Cadence Observations

\begin{tabular}{|c|c|c|c|c|c|c|}
\hline Cluster & Dates Observed & $\begin{array}{l}\text { Total } \Delta t^{\mathrm{a}} \\
\text { (d) }\end{array}$ & Typical No. Epochs & $\begin{array}{l}\text { Typical Min }(\Delta t)^{\mathrm{b}} \\
\text { (d) }\end{array}$ & $\begin{array}{l}\text { Typical Max }(\Delta t)^{\mathrm{b}} \\
\text { (d) }\end{array}$ & $\begin{array}{c}\text { No. Obj with } \geqslant 5 \text { Epochs, } \\
\text { Both Channels }\end{array}$ \\
\hline AFGL 490 & 2011 Oct $19-$ Nov 25 & 37.7 & 46 & 0.34 & 1.95 & $\sim 600$ \\
\hline NGC 1333 & 2011 Oct 12-Nov 14 & 33.5 & 72 & 0.04 & 1.95 & $\sim 240$ \\
\hline Orion $^{\mathrm{c}}$ & 2009 Oct $23-$ Dec 1 & 39.0 & 80 & 0.21 & 0.80 & $\sim 5200$ \\
\hline Mon R2 & 2010 Nov 8-Dec 23 & 44.4 & 46 & 0.26 & 2.67 & $\sim 240$ \\
\hline GGD 12-15 & 2010 Nov 16-Dec 24 & 38.5 & 78 & 0.01 & 1.82 & $\sim 340$ \\
\hline NGC $2264^{\mathrm{d}}$ & 2010 Nov 18-Dec 24 & 35.7 & 39 & 0.18 & 2.51 & $\sim 260$ \\
\hline L1688 & 2010 Apr 12-May 17 & 35.4 & 80 & 0.11 & 0.75 & $\sim 200$ \\
\hline Serpens Main & 2011 May 20-Jun 24 & 34.7 & 81 & 0.09 & 0.75 & $\sim 700$ \\
\hline Serpens South & 2011 May 20-Jun 24 & 34.7 & 81 & 0.09 & 0.75 & $\sim 370$ \\
\hline IRAS $20050+2720$ & 2010 Jun 12-Aug 9 & 57.8 & 99 & 0.06 & 3.68 & $\sim 690$ \\
\hline IC $1396 A^{c}$ & 2009 Sep 13-Nov 9 & 56.1 & 105 & 0.13 & 2.52 & $\sim 1480$ \\
\hline Ceph $C^{c}$ & 2010 Sep $18-O c t 30$ & 42.0 & 106 & 0.03 & 3.68 & $\sim 400$ \\
\hline
\end{tabular}

Notes.

${ }^{\text {a }}$ Total change in time, in days, between first and last fast cadence point.

${ }^{\mathrm{b}}$ Typical minimum or maximum change in time, in days, between adjacent points in the time series. Also see Figure 26 below, which has histograms of the time step distributions.

${ }^{\mathrm{c}}$ Additional observations exist in slower cadence.

${ }^{\mathrm{d}}$ Additional observations are part of CSI 2264. 


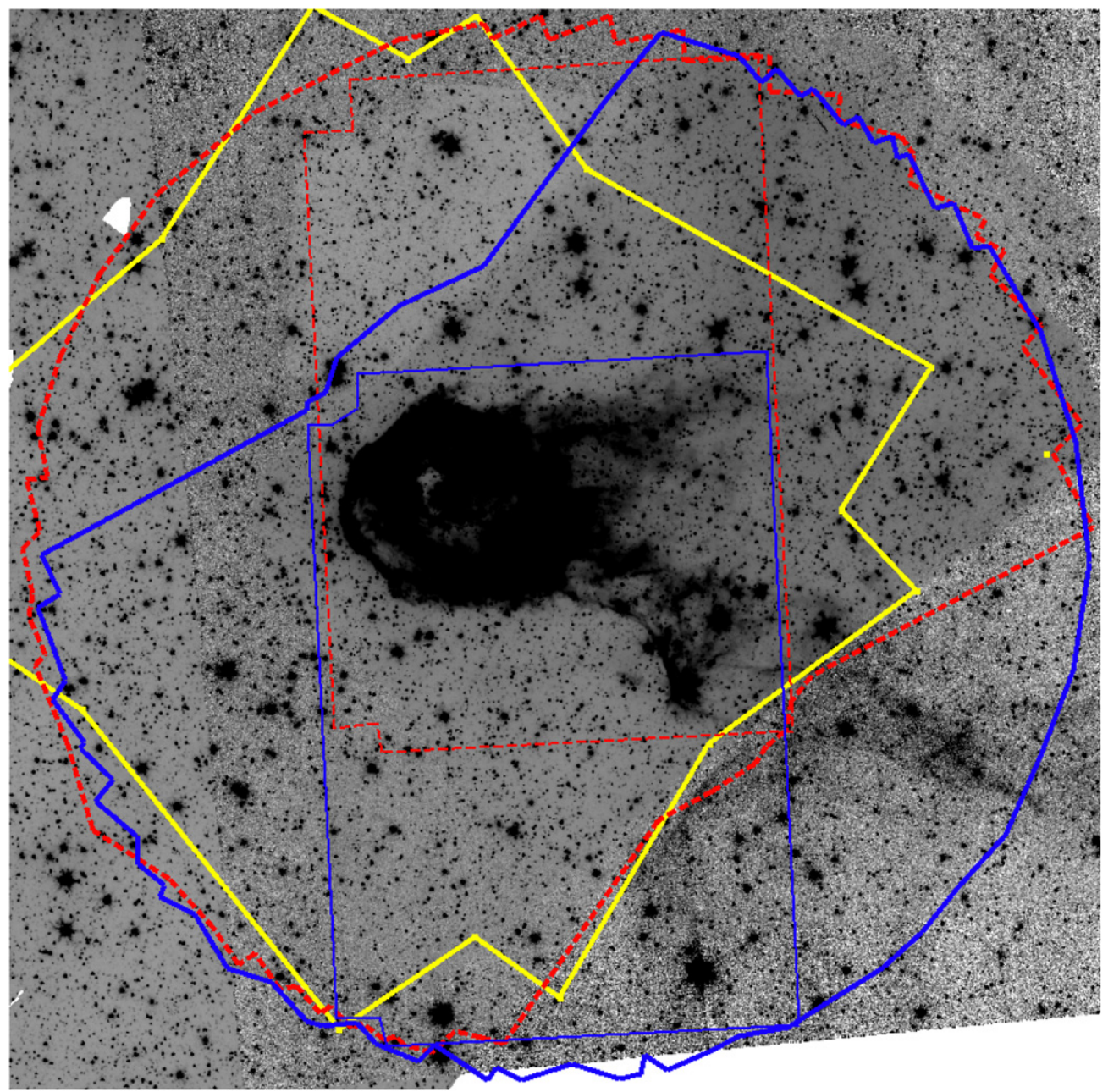

Figure 12. Same as Figure 2, but for our IC 1396A observations, with the north-south boundary extremes separated by $\sim 24^{\prime}$. As in Figure 5, a single epoch of observation is also indicated. Two pointings of Chandra data at two different roll angles were obtained here, resulting in the polygon of Chandra coverage.

(A color version of this figure is available in the online journal.)

Ceph $\mathrm{C}$ over its fast cadence window include those originally obtained as part of the original YSOVAR project, as well as data taken using a very similar distribution of time steps as part of a YSOVAR-related Chandra program; see Table 2. For consistency with the maximum timescales sampled in other clusters, we define the Ceph $\mathrm{C}$ fast cadence light curve to be a window spanning from 2010 September 19 through 42 days later, which is more representative of the fast cadence window in the other clusters. We note, however, that the sampling within this window is nonetheless enhanced relative to other smaller-field clusters, due to the inclusion of extra Spitzer observations obtained to support the coordinated Chandra observations. Two other clusters (IC 1396A and IRAS 20050) also sample longer timescales than the more typical $\sim 40$ day window (see Figure 27 below for an enlargement of the fast cadence). We did not truncate the last few slower cadence points from the IRAS 20050 time series because there are no other slower cadence observations, and truncating it would effectively "orphan" the last few points. The IC 1396A cadence does not provide a clean breakpoint, so it also has points covering a slightly longer window than "typical" included in its fast cadence (see Table 3 for the total time in the fast cadence for all clusters). IC 1396A was one of the first SFRs to be monitored intensively with Spitzer (MoralesCalderón et al. 2009), so it has additional cryo-era monitoring not shown in Figure 14. Serpens Main also has some cryo-era monitoring. GGD 12-15 and L1688 also have staring data, not included here.

\subsection{Warm Spitzer Observations: Data Reduction}

We started with the IDL package Cluster Grinder (G09), which has been used by several other projects (e.g., Megeath et al. 2012), and then made custom modifications to make it suitable for this time series data set. We started with the basic calibrated data (BCD) images released by the SSC. The BCD images used correspond largely to software version S18.18 (with about 20\% S19.0 and S19.1); earlier reductions such as those in MC11 used a mix of S18.12, 18.14, and 18.18. Each BCD frame was processed for standard bright source artifacts and combined into mosaics (at 0". 86 per pixel grid resolution) by exposure time (long or short), epoch, and bandpass. Cosmic ray hits and other transient artifacts are flagged during mosaic construction using redundant data at each pixel position in the final mosaic grid as a reference for the nominal value and allowable internal variation.

Since the HDR mode obtains a long and a short frame at each pointing, we need to combine data from the two exposures. HDR mosaics are merged together on a pixel-by-pixel basis, with appropriately scaled short-frame HDR values above 


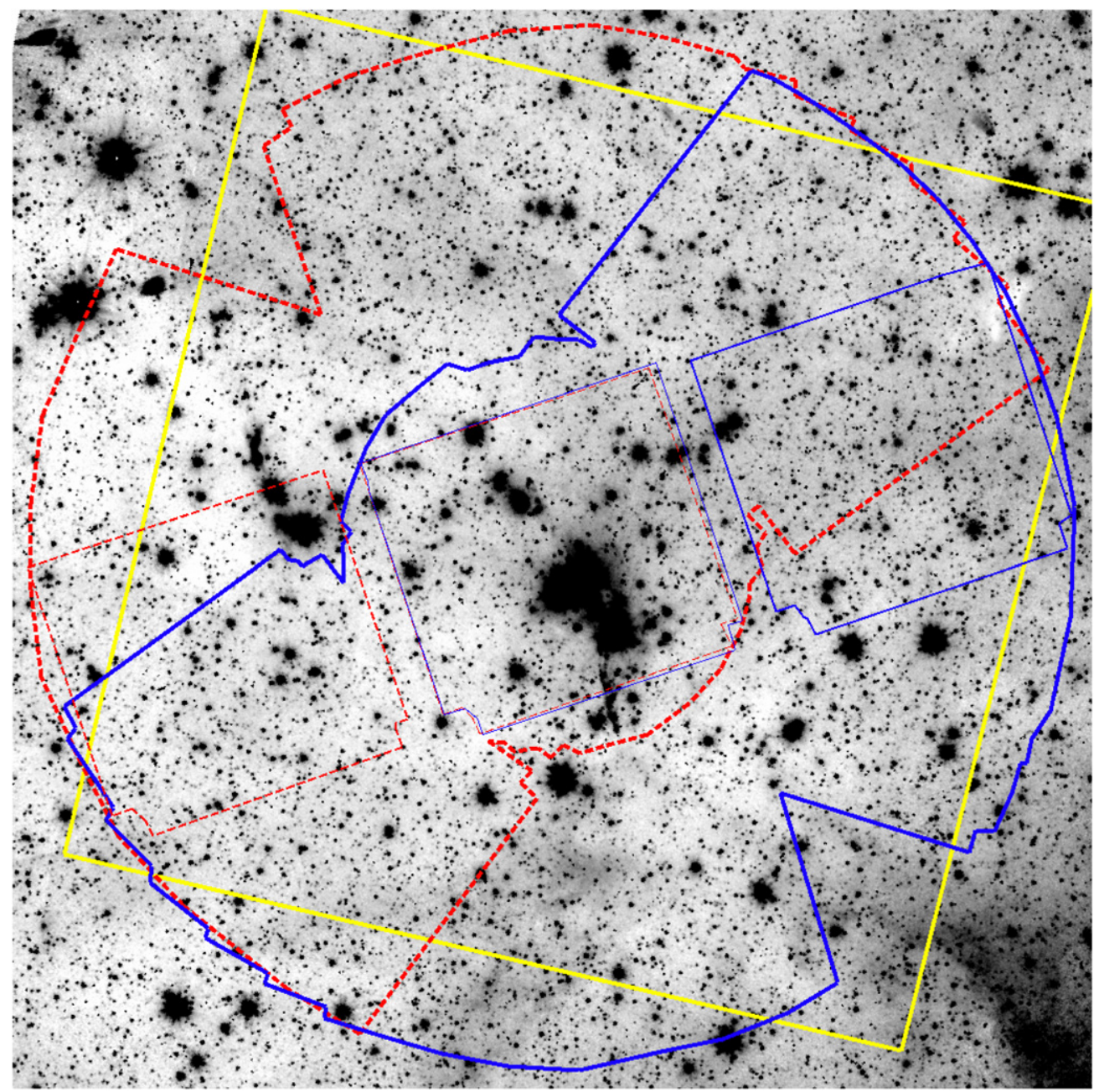

Figure 13. Same as Figure 2, but for our Ceph C observations, with the north-south boundary extremes separated by $\sim 20^{\prime}$. As in Figure 5, a single epoch of observation is also indicated. The field rotation here is quite significant.

(A color version of this figure is available in the online journal.)

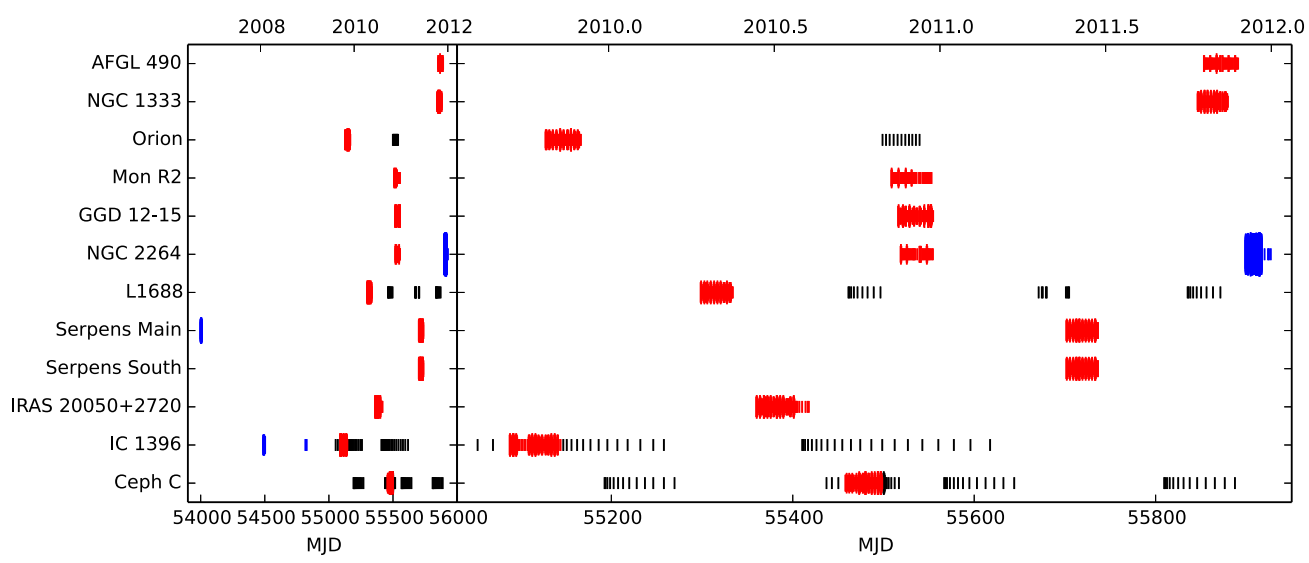

Figure 14. Visual overview of the observing seasons and the cadence for all clusters in the YSOVAR project. On the left side, all of the YSOVAR data are depicted (where Spitzer's cryogen ran out 2009 May 15, MJD 54966.925). On the right side is an expanded view of the data highlighted here and used for the "standard set for statistics" (see Section 3.2). The designated "fast cadence" observations are shown in red, with other YSOVAR-classic observations shown in black. Other YSOVAR-affiliated programs are shown in blue; the large post-cryo monitoring of NGC 2264 is CSI 2264, and the cryogenic observations of IC 1396A and Serpens are also shown. Each individual observation is marked by a "|" symbol in which the length of the line is proportional to the number of observations in a window $12 \mathrm{hr}$ before and after that particular observation. When the observations are so dense that the symbols overlap, the frequency of observations can thus be judged from the thickness of the line. An enlargement of the red, fast-cadence sequences can be found in Figure 27 below.

(A color version of this figure is available in the online journal.) 
a set threshold (individual pixel values corresponding to sources $\sim 10$ th mag, dependent on background level) replacing significantly compromised pixels in the long-frame HDR mosaic.

Point source detection and aperture photometry are then performed for each channel and each AOR/epoch. The aperture radius was 2 ". 4 , with a sky annulus having inner and outer radii of 2.' 4 and 7 ". 2, respectively. We adopted Vega standard magnitude zero points of 19.30 and 18.64 for $1 \mathrm{DN} \mathrm{s}^{-1}$ total flux at 3.6 and $4.5 \mu \mathrm{m}$, respectively. These values include standard corrections for our chosen aperture and sky annulus sizes (Reach et al. 2005; Carey et al. 2010).

Extensive tests were run on aperture photometry obtained in three different ways. Our initial approach was to obtain photometry from the mosaic created for each AOR, with the assigned time for that point being the average time for the AOR. We also obtained photometry on each BCD frame individually, with the individual, specific time of that observation being assigned to that point. Finally, we took the set of all individual BCD measurements for a given source within a given AOR, and took the mean brightness and the mean time for that set of observations. This latter approach proved to have the best quality photometry. It facilitated the treatment of two wellknown systematic effects (residual gain and pixel phase effects) while keeping our signal-to-noise ratios (S/Ns) close to ideal. It provided the highest $\mathrm{S} / \mathrm{N}$ light curves, even if the time resolution is slightly lower than what might be theoretically possible. Effectively, this choice means that a $\mathrm{S} / \mathrm{N} \sim 5$ is required for a viable source in a given $B C D$, such that the net $S / N$ of the source over the entire AOR is at least $\sim 10$. If a source fell on the edge of the BCD such that the two native pixel radius was not entirely within a given $\mathrm{BCD}$, no measurement is obtained. Measurements were also excluded as non-viable if the measurement (in the aperture or annulus) included a masked IRAC pixel such that obtaining a finite measurement was not possible, or if the source was faint enough (or the sky variation high enough) that the net measured flux was $<0$. If there were three or more valid detections of a given source in a given AOR, we could perform outlier rejection, and outliers were flagged; the rest of the measurements were combined by uniform weight arithmetic mean. The uncertainties were added in quadrature and divided by the included measurement count.

Source matching between epochs and wavelengths was performed by position, taking the nearest source within $1^{\prime \prime}$. Final positions for each source were the mean of the individual measurements. Our astrometric residuals as compared to 2MASS suggest an uncertainty of $<200$ mas root-mean-square (rms), after a single iteration refinement of the astrometry using a first set of mosaics constructed blindly with the BCD-delivered world coordinate system (WCS). Objects with fewer than five viable detections over separate epochs in a single band were not retained as valid light curves.

We note here that there are some residual instrumental column pulldown effects (see the IRAC Instrument Handbook for more information) in some areas near bright sources. This is a more significant problem for clusters with a large number of very bright sources (e.g., Mon R2; see brightnesses in Figures 1-13). The locations of these artifacts can change with time, particularly in fields at high ecliptic latitude and thus with significant field rotation during a given visibility window (see Section 2.3), and can have particularly significant effects on sources fainter than $\sim 12$ th mag. Objects falling in such regions that are faint and that had non-repeating structure in their light curves should be particularly scrutinized (including visual inspection of the input frames) for the validity of their light curves. Points obviously compromised by these effects should additionally be identified as non-viable points and rejected. Discussions of this process for individual objects will appear in the clusters papers to follow.

Even after all of the processing to this point, some occasional outliers in the light curves remain. To identify outliers within the fast cadence data, we initially identified points several $\sigma$ away from the mean for each light curve. We used this approach in MC11, with a different (much earlier) processing of the original Spitzer data. However, our more recent, improved processing described here reduced the number of outliers. We experimented with algorithms for rejecting outliers from each light curve, but were unable to identify a technique that rejected visually spurious points while preserving apparent bona fide variability. An approach as we used in MC11 would, for example, exclude points at the beginning and end of a light curve with a long trend. Our estimates suggest that implementing an outlier rejection strategy affects $\lesssim 10 \%$ of the light curves tagged as intrinsically variable (as per methods described further below), so we retained all points in the light curves. Outliers may still be identified in a few specific individual cases; those outliers are then effectively excluded from the light curve, and are discussed when relevant.

\subsection{Warm Spitzer Observations: Noise Floor}

Many tests of variability (e.g., a $\chi^{2}$ test; see Section 5.2) depend on accurate error estimates for each data point in the time series. Initial estimates of uncertainties were derived by combining three terms in quadrature (see also the discussion in Megeath et al. 2004): shot noise in the aperture, shot noise in the mean background flux per pixel integrated over the aperture, and the standard deviation of the sky annulus pixels to account for the influence of non-uniform nebulous background. Such error values proved to be a slight underestimate of the true uncertainty, and do not take into account a "floor" in the errors from calibration errors, primarily the intrapixel gain variation.

We wanted to find a value for this floor that worked for all clusters (since all of the mapping observations discussed here were obtained in the same way). We also wanted to be conservative in that we wanted the set of objects we selected as variable at the end of this process to be reliably variable (with very few, if any, non-variables selected as variable), even if it meant that our sample would not be complete in that some legitimately (lower level) variable objects would not be selected. In other words, we wished to err slightly on the side of overestimating errors. We investigated the rms error for each light curve for each object versus magnitude and versus the median photometric uncertainty for all sources. In MC11, we estimated the true error as a function of magnitude to be singlevalued as a function of magnitude bin. In general, this means that the errors will be correct on average, but there will be specific objects (e.g., those in high background regions) for which they are too low.

Here, we aimed to improve on this estimate by finding a value for the noise floor that can be added in quadrature to the error estimates for each point, preserving the errors obtained for each point, localized in time and space. For each channel, for every object in each cluster having at least 20 epochs in its light curve so as to ensure well-determined values, we plotted the measured rms $(\sigma)$ of the light curve against its median photometric uncertainty; see Figure 15. Orion alone represents about one-quarter of the light curves shown in Figure 15, so it 


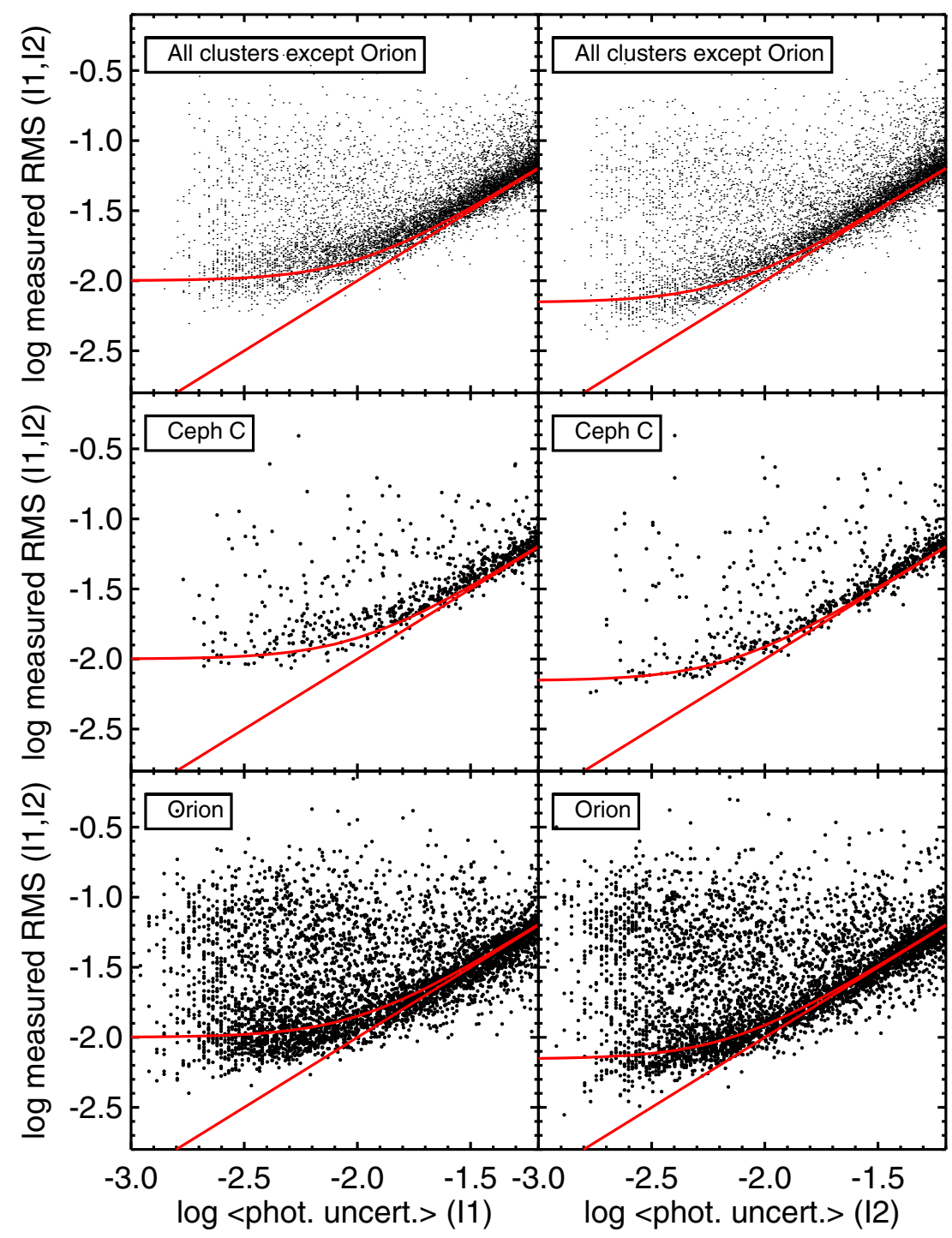

Figure 15. Plots of measured rms $(\sigma)$ vs. median photometric uncertainty for I1 (left) and I2 (right). The top row is for all clusters except Orion, the middle row is Ceph C only, and the bottom row is Orion only. The straight red line is the one-to-one relationship between these parameters, e.g., the expected relationship if there was no "noise floor"; the curved red line is the empirically derived curve which we used to determine a noise "floor" of 0.01 mag for I1 and 0.007 mag for I2, which we then added in quadrature to the individual errors obtained for each point. To appear in this plot, objects must have more than 20 epochs. Ceph C appears separately to give an indication of what these plots look like for individual clusters. We combined all the clusters together to better determine the empirical floor, even though there are some variations among the clusters; see the text.

(A color version of this figure is available in the online journal.)

is useful in some contexts to separate Orion from the analysis of the rest of the ensemble. Data for an individual smaller-field cluster are thus shown in the figure for reference.

The largest-amplitude sources in Figure 15 are true variables. To determine the noise floor, we are interested in how the observed rms for non-variables over many epochs compares to the formal errors. The bulk of the distribution of points in Figure 15, e.g., the non-variable and less noisy sources in the ensemble of points, fall in the same region in each plot; the distributions run smoothly down to an asymptotic value. However, there are three components contributing to the overall scatter of the regions with more objects seen in Figure 15, where they are not necessarily seen as distinct features. As we move to brighter objects (generally those with smaller median photometric uncertainty), we have a statistically larger chance that the objects will be cluster members, e.g., legitimately young and therefore expected to have a greater likelihood of being highly variable. There is a transition between the short and long
HDR frames (at $\sim 10$ th mag) which affects the measured error for sources that are faint in the short frames but not in the long frames. There are variations in depth ( $\sim 16$ th -17 th mag) reached across clusters due to variable and nebulous backgrounds (the variations in depth can also be seen in the faint limits of Figures 19 and 20, discussed below) such that a given median photometric uncertainty need not necessarily refer to stars of roughly the same brightness among the clusters. Figure 15 includes an individual cluster (Ceph C) to give an indication of this cluster-dependent variation in the floor; it can be seen that fewer points fall below the red line on the left side of each panel in Ceph $\mathrm{C}$ than they do in the Orion plots on the bottom row.

Close inspection of Figure 15 reveals that the asymptotic noise floor value is on average slightly higher for the $3.6 \mu \mathrm{m}$ channel and slightly lower for the $4.5 \mu \mathrm{m}$ channel. This is consistent with expectations that the dominant source of error in the asymptotic noise floor is from residual intrapixel gain variations, since the intrapixel gain effect is lower in I2 than in I1. There are also 


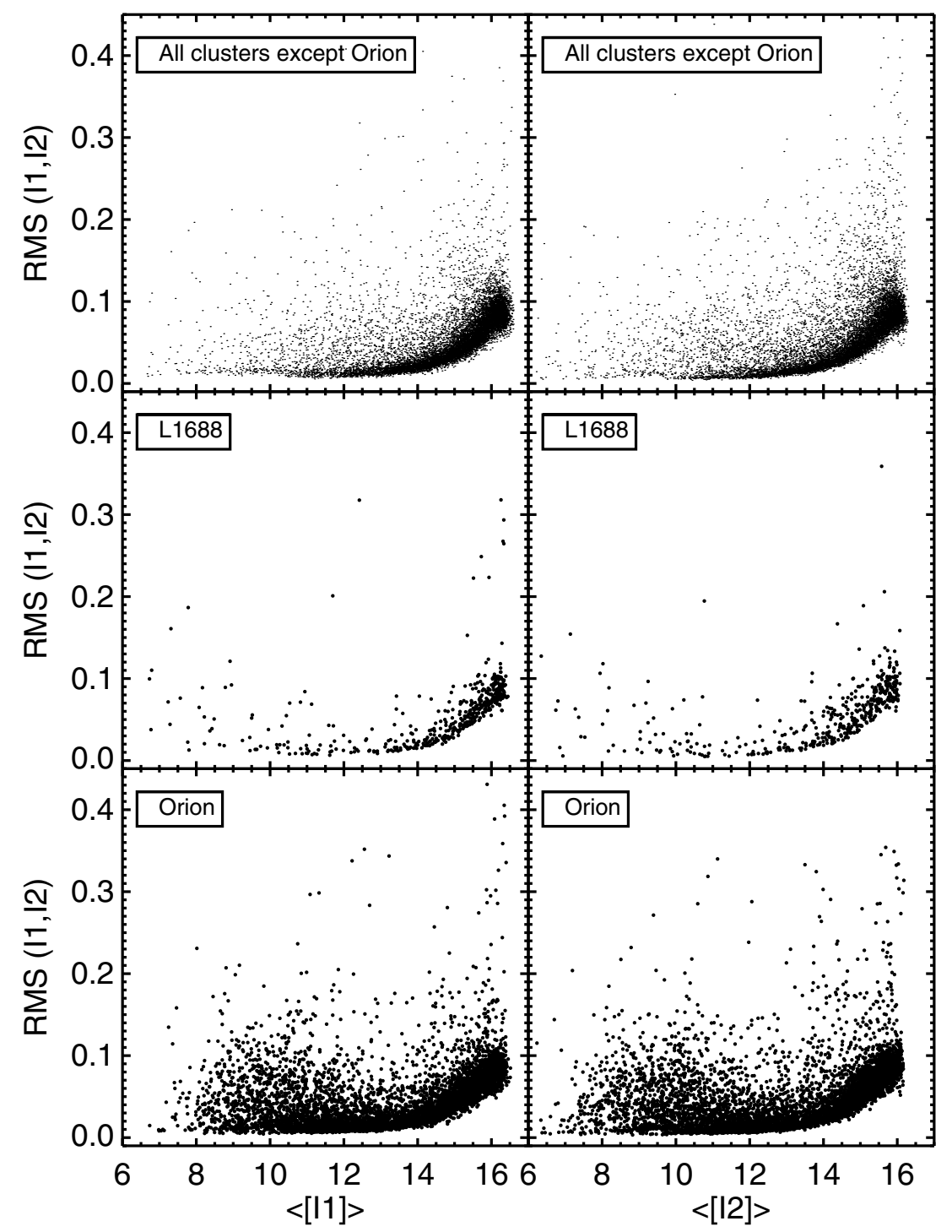

Figure 16. Plots of measured rms $(\sigma)$ vs. mean magnitude for I1 (left) and I2 (right). The top row is for all clusters except Orion, the middle row is L1688 only, and the bottom row is Orion only. There is more, and more significant, variation among the brighter sources. (Some very large rms values fall above the range shown in this plot; the range is limited here for clarity.)

more points with significantly larger $\sigma$ in the $4.5 \mu \mathrm{m}$ channel. The $4.5 \mu \mathrm{m}$ channel has more contributed noise in any given light curve - shocks and scattered light, which are very common in our target regions, contribute to the overall scatter in $\sigma$ at $4.5 \mu \mathrm{m}$. There is also some lower-level contribution from polycyclic aromatic hydrocarbon (PAH) features (also common in these regions) in $3.6 \mu \mathrm{m}$ (see, e.g., Flagey et al. 2006). These contributions to both channels mean that there is some cluster-tocluster variation in the uncertainties associated with individual sources at a given magnitude.

We determined that a systematic error of $0.01 \mathrm{mag}$ in I1 and $0.007 \mathrm{mag}$ in I2 (both indicated in Figure 15) provides the best fit to the photometric rms seen in each channel in that it is a conservative representation of the distribution as the distributions approach the asymptote. These values are also quite comparable to the magnitude of the intrapixel gain effect in each of these channels, and it is not expected that the errors in IRAC photometry from mapping observations would be less than $0.1 \%$. Very similar values are obtained via a slightly different approach for the CSI 2264 mapping in Cody et al. (2014), where the populations of members and non-members are better understood. We added our empirically derived systematic values in quadrature to each of the individual errors obtained by our pipeline.

Figure 16 plots the light curve rms $(\sigma)$ derived from the corrected light curves against the mean magnitude for that light curve. Here, again, one can see more, and more significant, variation among the brighter sources. Relatively few sources have much larger uncertainties because they are close to bright neighbors, chip edges, or affected by other instrumental artifacts; many of the sources with large rms are legitimately variable. For sources brighter than 13th mag, the total uncertainty is dominated by the error floor. The overall noise increases significantly fainter than about 14 th magnitude, and the rapid falloff of the number of objects detected in the survey beyond 16th magnitude is immediately apparent (see also Figures 19 and 20 below). There are small variations between clusters, which are caused by different levels of diffuse emission and a different degree of instrumental artifacts from, e.g., residual pull-down. A sample individual cluster is included in Figure 16 as an indication of what is seen in the individual smaller-field clusters. 
Table 4

Summary of Cryo-epoch Spitzer Observations

\begin{tabular}{|c|c|c|c|c|c|}
\hline Cluster & AORKEY ${ }^{\mathrm{a}}$ & Obs. Date & Adop. Date ${ }^{b}$ & $\Delta(t)^{\mathrm{c}}$ & Notes \\
\hline AFGL 490 & 3654656,3663104 & 2004 Oct 8, 2004 Sep 24 & 2004.9 & 6.9 & Reduction from G09 \\
\hline NGC 1333 & $3652864,5793280,4316672$ & 2004 Feb 10, 2004 Sep 8, 2004 Feb 3 & 2004.5 & 7.3 & $\begin{array}{l}\text { Reduction from G09, Gutermuth } \\
\text { et al. (2008b); some data from } \\
\text { c2d included where necessary }\end{array}$ \\
\hline Orion & (Many) & 2004 Mar-2004 Oct & 2004.5 & 5.9 & $\begin{array}{l}\text { Reduction from Megeath et al. } \\
\text { (2012) }\end{array}$ \\
\hline Mon R2 & 3659008,3668224 & 2004 Mar 13, 2005 Apr 7 & 2004.3 & 6.6 & Reduction from G09 \\
\hline GGD 12-15 & 3658752,3667968 & 2004 Mar 31, 2004 Mar 15 & 2004.3 & 6.6 & Reduction from G09 \\
\hline NGC 2264 & $\begin{array}{l}3956480,3956736,3956992, \\
3957248\end{array}$ & $\begin{array}{l}2004 \text { Mar 6, } 2004 \text { Oct 8, } 2004 \text { Mar 6, } \\
2004 \text { Oct } 8\end{array}$ & 2004.5 & 6.4 & Re-reduced in G09 style \\
\hline L1688 & 3652096,4321280 & 2004 Mar 7, 2004 Aug 26 & 2004.3 & 6.7 & $\begin{array}{l}\text { Reduction from G09; some data } \\
\text { from c } 2 \text { d included where } \\
\text { necessary }\end{array}$ \\
\hline Serpens Main & $3652352,3661568,12649216$ & 2004 Apr 1, 2004 Apr 6, 2005 Apr 12 & 2004.3 & 7.2 & $\begin{array}{l}\text { Reduction from G09; some data } \\
\text { from c } 2 \text { d included where } \\
\text { necessary }\end{array}$ \\
\hline Serpens South & $\begin{array}{l}\text { 19957248, 19958016, 19998464, } \\
\text { 19998720, 20002304, 20002560, } \\
\text { 20018688, 20018944 }\end{array}$ & $\begin{array}{l}2006 \text { Oct } 27,2006 \text { Oct } 27,2006 \text { Oct } \\
28,2006 \text { Oct } 28,2006 \text { Oct } 27,2006 \\
\text { Oct } 27,2006 \text { Oct } 28,2006 \text { Oct } 28\end{array}$ & 2006.9 & 4.6 & $\begin{array}{l}\text { Reduction from Gutermuth et al. } \\
\text { (2008b) }\end{array}$ \\
\hline $\begin{array}{l}\text { IRAS } \\
20050+2720\end{array}$ & 3656448,3665152 & 2004 Jun 9, 2004 Sep 25 & 2004.5 & 6.1 & Reduction from G09 \\
\hline IC 1396A & 3959040,4316416 & 2003 Dec 20, 2004 Jun 23 & 2004.0 & 6.4 & Re-reduced in G09 style \\
\hline Ceph C & 3655936,3664384 & 2003 Dec 19, 2003 Dec 19 & 2004.0 & 6.4 & Reduction from G09 \\
\hline
\end{tabular}

Notes.

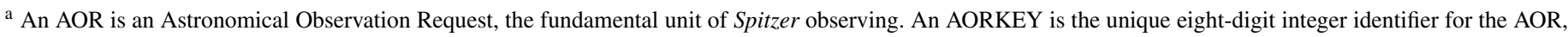
which can be used to retrieve these specific data from the Spitzer Heritage Archive.

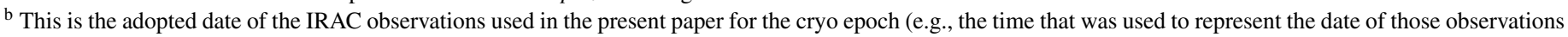

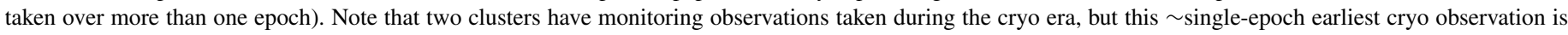
what is used in the present paper as the "cryo epoch" for comparison to the "YSOVAR epoch."

c $\Delta(t)$, in years, between adopted cryo epoch and mean YSOVAR fast-cadence time.

\subsection{Cryogenic-era Spitzer Data}

Early in the Spitzer mission, each of our 12 regions was observed, often as part of the guaranteed time observations or the original Cores-to-Disks (c2d) Legacy program (Evans et al. 2003, 2009b); these observations are summarized in Table 4. In most cases, the data were obtained at two epochs. For clusters near the ecliptic plane, asteroids that happen to be passing through the region at the time of observation appear as long wavelength sources that can resemble embedded lowmass YSOs. Thus, the two epochs were often planned to be separated by a time of the order of hours (e.g., the earliest epochs of Serpens, much of L1688) to allow the moving solar system objects enough time to move, and the corresponding pixels to be rejected as outliers when the individual frames were combined. For some observations (e.g., Orion), to facilitate bright source artifact mitigation, the epochs were separated by $\sim 6$ months such that the orientation of the arrays in the later observation is $180^{\circ}$ from that in the early observation (see Section 2.3 above). For the most robust detections, the cryogenic data can be combined into a single early epoch, or one can compare the two epochs to constrain variability on timescales of months (e.g., Megeath et al. 2012 for Orion at IRAC bands) or hours (e.g., Rebull et al. 2007 for Perseus at $24 \mu \mathrm{m}$ ).

To retain information from the individual epochs, the cryogenic data for all of our clusters were reduced identically to the YSOVAR monitoring data, except using cryogenic calibrations (e.g., the zero point and threshold between the short and long HDR frames). Source detection was performed independently in each band for each epoch. However, for reasons we now de- scribe, measurements derived from individual epochs from the cryogenic era cannot be used generally over the entire region, and the identical approach adopted for the rest of the YSOVAR data cannot be used generally for the cyrogenic data.

For the YSOVAR data, each epoch covered the region of interest with sufficient redundancy, so that data reduction can be performed on a per-epoch basis as described in Section 2.5 above. For some of the cryogenic era data, the observations were designed to have sufficient redundancy in the region of interest only once all of the cryogenic era observations were combined. Specifically, for some of those clusters that were observed in two epochs, for moving object identification, the observations from a single epoch were not designed to robustly measure objects at that epoch; it was envisioned that the two epochs would be combined to create a net mosaic of the inertial targets. For example, one epoch might have two frames with another two frames at a later epoch. Combining those after the fact provides four frames of dithered observations to remove artifacts and moving objects. However, a single epoch, having only two frames, does not necessarily have enough data for robust photometry. A complication comes from the combination of individual frames to compose a map at a given epoch. The maps at any one epoch are constructed from multiple pointings, with overlap between each pointing. Therefore, in those thin regions between adjacent pointings, there are more than two frames at a given epoch. Because our pipeline requires at least three frames per position, it only derives (and archives) highquality photometry at either of those two epochs for that thin region between adjacent pointings in the map, not the entire map at that epoch. Therefore, if one downloads the earliest epoch data 
from our archive for those clusters, one obtains a "grid-shaped web" of viable data, not complete coverage of the region. We have retained all of these individual epoch data in the data we deliver to IRSA because it may be useful to future researchers to have individual measurements of sources serendipitously falling in those regions. However, for users not aware of this "feature," it will seem strange to only have cryogenic data over a web of coverage in those early epochs.

In the context of the present paper, therefore, all of the earliest cryogenic-era Spitzer observations were combined into one mosaic per epoch per channel, which was then run through Cluster Grinder. The time assigned to this early epoch of observation is the average time of all of the observations that went into the mosaic, summarized in Table 4.

As previously stated, IC 1396A and Serpens Main were explicitly monitored in the cryogenic era with Spitzer, and these data were also reprocessed, retaining individual epochs of observation.

The data were bandmerged across Spitzer bands by position, within a search radius of $1^{\prime \prime}$. (Recall that the IRAC photometry apertures we used were 2.4.)

Gutermuth et al. (2008b, 2009, 2010) present methodology for identifying YSOs from the cryogenic catalog. The details of the selection process appear in those papers, but in summary, multiple cuts in multiple color-color and color-magnitude diagrams are used to identify YSO candidates, as distinct from, e.g., extragalactic and nebular contamination. This color selection is part of Cluster Grinder, and thus we have this classification, based on the cryogenic data, for all of our clusters; we used it in Section 2.1.2 and in part of Table 1. We have adopted this YSO selection mechanism as part of one of the primary YSOVAR sample definitions; see Section 3.

\subsection{New Data at Optical and NIR Wavelengths}

For completeness, we note here that, in addition to the new IRAC data, we often also obtained contemporaneous optical and NIR observations from the ground using a variety of telescopes. The details of each telescope/camera and set of accompanying observations is beyond the scope of this paper, and is (or will be) provided in each paper discussing the results from each cluster. For example, MC11 discussed observations obtained from four other ground-based telescopes obtained in 2009-2010, contemporaneous with the first epoch of Orion observations; Cody et al. (2014) discussed observations obtained contemporaneously with the CSI 2264 campaign. Because these ancillary monitoring data are different for each cluster, we omit them here for clarity.

\subsection{Other IR Archival Data}

We also included $2 \mathrm{MASS} J H K_{s}$ data for all of our clusters. For two clusters (NGC 1333, L1688), deeper than survey 2MASS data (the $6 \times$ data) are available from the 2MASS archive, and are included where available. Such detections were also bandmerged to the rest of the catalog by position, within a search radius of $1^{\prime \prime}$.

For three of our clusters (L1688, Mon R2, Serpens Main), deeper NIR data from the UKIRT Infrared Deep Sky Survey (UKIDSS; Lawrence et al. 2007) broadband data were publicly available, but not necessarily at all or even most of the UKIDSS bands $(Z, Y, J, H, K)$; in all three cases, there were at least $K$-band data, which is important for our calculation of SED class (see Appendix B). Where available, these data are also bandmerged to the rest of the catalog by position, within a search radius of $1^{\prime \prime}$. Details of which bands are available and to what depth will be included in the individual papers associated with the relevant clusters.

For two of our clusters, AFGL 490 and Ceph C, data in two Spitzer bands are available from one of the warm portions of the Spitzer program called the Galactic Legacy Infrared Mid-Plane Survey Extraordinaire (Benjamin et al. 2003). Where available, those single-epoch, shallow data were also bandmerged to the rest of the catalog by position, within a search radius of $1^{\prime \prime}$. Since those measurements are independent measures of these objects at [3.6] and [4.5], these measurements were retained as distinct points from the cryo [3.6] and [4.5], or the means of our light curves.

For three other clusters, NGC 1333, L1688, and Serpens Main, data are available from the c2d (Evans et al. 2003, 2009 b) program data deliveries. The data used for these deliveries are typically the same BCDs as were used in the cryogenic data (Section 2.7). As such, then, they are not independent measurements, and these data were only used to supplement our cryogenic-era catalog if a band was missing, e.g., because G09 had identified it as having insufficient $\mathrm{S} / \mathrm{N}$ in that reduction.

Naturally, each cluster has different amounts of additional data in the literature, and the details of exactly which data are included (and how it was merged to the rest of the catalog) will appear in the corresponding YSOVAR cluster paper.

\subsection{Chandra Data}

X-ray observations are an excellent complement to mid- and near-IR observations in regions of star formation. Due to the strong correlation between X-ray luminosity and age, X-ray emission is particularly effective in identifying YSOs that are free of IR excess (Class IIIs), such that X-ray surveys provide samples of young stars unbiased by their IR characteristics.

X-ray observations can penetrate up to $A_{V} \sim 500$ mag into a star forming cloud with very deep integrations (Grosso et al. 2005). However, even with shallow integrations, one can reach as deep or deeper in the X-rays than many NIR surveys in the JHK bands. The Chandra X-ray Observatory, with its high angular resolution mirrors and low-noise detectors, is particularly effective in resolving crowded fields down to 0 '.5 scales. Furthermore, in X-rays, OB stars are often not much brighter than pre-main-sequence stars, so close companions, even when associated with OB stars, can be identified (Stelzer et al. 2005). Chandra data (or any other X-ray data, e.g., from the European Space Agency's XMM-Newton observatory) and Spitzer data combined have been shown to yield a more complete survey of members; there are many examples of this in the literature. For some of the clusters in our target list, an incomplete listing of such work could include Winston et al. (2007, 2010) for NGC 1333 and Serpens Main, respectively, Günther et al. (2012) for IRAS 20050, Getman et al. (2006) for IC 1396A, or Imanishi et al. (2001) for L1688.

Identification of additional cluster members is the primary purpose for which we include X-ray data, where available, for the YSOVAR clusters. We can then compare variability characteristics for the X-ray-detected sample with that from, e.g., the IR-selected sample. With the exception of AFGL 490, ${ }^{32}$ all of the YSOVAR clusters have been observed by Chandra using the Advanced CCD Imaging Spectrometer for wide-field

\footnotetext{
32 As of early 2014, AFGL 490 has not been observed by XMM-Newton either.
} 
imaging (ACIS-I), and nearly all of them have already been published. The date, duration, and aimpoints of these observations are listed in Table 5, along with citations to the literature where possible; footprints of the observations are included in Figures 2-13.

In order to create a more unified set of detection criteria, and in order to reach fainter sources, X-ray data for the nine smallerfield clusters with X-ray data were reprocessed to achieve internal consistency and maximize source detection. We used Chandra pipeline DS 8.4.5. Detailed X-ray data and analysis have already been published for the very deep observation of the ONC (Feigelson et al. 2005; Getman et al. 2005), and for two shallower fields north and south of the ONC (Ramirez et al. 2004b); similarly, for NGC 2264, Flaccomio et al. (2006) and Ramirez et al. (2004a) have published deep Chandra data (our region here is primarily covered by Flaccomio et al. 2006). Because those two regions include X-ray data substantially deeper than those for the rest of the clusters, we have not reprocessed these X-ray data here, but instead taken those source lists from the literature. (A primary reason we reprocessed the nine smaller-field clusters is to reach fainter sources, which the deeper integrations in Orion and NGC 2264 already accomplish.) Additional X-ray data obtained contemporaneously with Spitzer monitoring will be discussed in the appropriate cluster-specific papers.

The region with ACIS-I data is usually smaller than the full region observed with Spitzer during the cryogenic era, but often covers the region monitored for YSOVAR; see Figures 2-13. Aside from AFGL 490 (which has no X-ray data), the region with the least spatial Chandra fractional coverage is Orion; the Orion region with IR light curves is by far the largest of our sample. The other 10 clusters have complete or nearly complete $\mathrm{X}$-ray coverage of the region with two-band IRAC light curves; $\mathrm{X}$-ray coverage of the regions with light curves in only oneband varies. The Chandra sensitivity varies across the FOV, with higher sensitivity in the center of the pointing, decreasing toward the edges.

Source detection from the archival Chandra data is performed with the wavdetect algorithm in CIAO (Chandra Interactive Analysis of Observations; Fruscione et al. 2006), versions 4.5 and 4.6. The details for the detection process used here are identical to those used in Günther et al. (2012). Our chief goal is identification of all X-ray sources, since even a weak detection-provided it is coincident with an IRAC source-has a high likelihood of being a legitimate source, though this does not necessarily prove cluster membership. Table 6 lists the number of X-ray sources detected above the $2 \sigma$ significance level in the entire ACIS-I field (a single ACIS-I FOV is $\sim 16^{\prime} \times \sim 16^{\prime}$ ). For comparison, Table 6 also indicates the number of detections reported in the literature, often for exactly the same data. We recover essentially all the previously reported sources, and add a significant number of weaker ones due to the lower threshold employed here. Note that without the additional criterion imposed here of requiring a match between an X-ray source and an IRAC source, the $2 \sigma$ significance level used here would be too low and would result in a high number (about onethird) of spurious sources. The requirement of a positional match with an IRAC source allows us to use such a low threshold. We found that below $2 \sigma$, we do not find matches between $\mathrm{X}$-ray and IR sources in excess of matches expected due to random chance.

To determine if a given X-ray source is coincident with an IRAC source, we matched X-ray sources to IRAC source lists generated for Spitzer cryogenic-era observations, as described in Section 2.7 above. Due to the highly spatially dependent Chandra point-spread function, three matching radii were used. For sources within $3^{\prime}$ of the aimpoint, the matching radius was $1^{\prime \prime}$. For sources more than $6^{\prime}$ off-axis from the aimpoint, the matching radius was $2^{\prime \prime}$. In between, the matching radius was 1 1".5. We note that IRAS $20050+2720$ and IC 1396 A are both exceptions since the fields were each observed multiple times, at different roll angles. Since IRAS $20050+2720$ covered a wide range of rotation angles, in that case, the source positions are composites of different point spread functions; matches were made more carefully in these observations. For IC 1396A, the range of roll angles was smaller, so the same approach was used as for the rest of the clusters. For each cluster, in Table 6, we list the total number of X-ray sources with a match to an IRAC source within the appropriate radius. We note here for completeness that there are some very bright X-ray sources without IR counterparts; for our purposes within YSOVAR, we exclude these sources.

If an X-ray source is bright enough for spectral fitting, one can determine four key characteristics: X-ray temperature, gas absorption, mean flux $\left(F_{x}\right)$, and variability. We fit a single Astrophysical Plasma Emission Code (APEC) thermal emission model (Smith et al. 2001) for each source detected above 30 net counts, using $\mathrm{C}$-statistics and leaving the absorbing column density $\left(N_{\mathrm{H}}\right)$, the temperature $(T)$, and the volume emission measure as free parameters to determine the first three of the above characteristics. The metallicity is fixed at $30 \%$ of the solar abundances (Anders \& Grevesse 1989), in keeping with typical values from coronal emission from late-type stars. Details of the extraction and fitting processes are similar to those discussed by Winston et al. (2010), which follows the procedure for automated processing laid out for the ANCHORS (AN archive of CHandra Observations of Regions of Star formation) pipeline (Spitzbart et al. 2005). The key point is that all fits are done assuming that the source is a star with a thermal one-temperature spectrum. We calculate luminosities $\left(L_{x}\right)$ for each source using the fluxes $\left(F_{x}\right)$ from $0.3 \mathrm{keV}$ to $8.0 \mathrm{keV}$ and line-of-sight absorptions, assuming the distance to each cluster given in Table 1. Detailed flux errors due to the fit were not calculated separately for each source, since systematics are likely to dominate; instead, global flux errors were determined using the CIAO tool dmextract. This process calculates a simple error estimate based on photon statistics and the mean value of the exposure map in the source region. These errors are about $4 \%$ at 2000 counts, about $35 \%$ at 100 counts, and the errors reach $100 \%$ below about 50 counts. The error budget is dominated by photon counts and uncertainty in $N_{\mathrm{H}}$. There is no evidence that such errors are markedly biased by the C-statistic.

Since errors on $L_{x}$ can be dominated by systematic effects, we have reprocessed all sources (in those nine smaller-field clusters with Chandra data), even those with previously published fluxes, to ensure uniform spectral fitting methodology. For faint sources with fewer than 30 counts, no fit can be performed. In this case, we determine a median photon energy, which, when combined with the count rate, leads to an approximate flux determination. All spectral properties presented here are effectively time averaged over all observations.

To determine the fourth of the characteristics above (variability), we tested the light curves for variability using the Gregory-Loredo method (GL-vary; Gregory \& Loredo 1992). This method uses maximum-likelihood statistics and evaluates a large number of possible break points from the prediction of 
Table 5

Summary of X-ray Observations

\begin{tabular}{|c|c|c|c|c|c|}
\hline Cluster & Aimpoint $^{\mathrm{a}}(\mathrm{J} 2000)$ & Obsid & Exp. Time (ks) & Obs Date & Notes \\
\hline AFGL 490 & $\ldots$ & $\ldots$ & $\ldots$ & $\ldots$ & No X-ray observations \\
\hline NGC 1333 & $03: 29: 05.60,+31: 19: 19.00$ & 642 & 43.2 & $2000 \mathrm{Jul} 12$ & $\begin{array}{l}\text { See also analysis by Getman et al. (2002), } \\
\text { Winston et al. (2010) }\end{array}$ \\
\hline NGC 1333 & $03: 29: 02.00,+31: 20: 54.00$ & 6436 & 39.5 & 2006 Jul 5 & (As above) \\
\hline NGC 1333 & $03: 29: 02.00,+31: 20: 54.00$ & 6437 & 36.6 & 2006 Jul 11 & (As above) \\
\hline Orion & $\cdots$ & $\ldots$ & $\cdots$ & $\ldots$ & $\begin{array}{l}\text { Reanalysis beyond the scope of this paper; } \\
\text { values taken from Getman et al. (2005) and } \\
\text { Ramirez et al. (2004b) }\end{array}$ \\
\hline Mon R2 & $06: 07: 49.50,-06: 22: 54.70$ & 1882 & 98.1 & 2000 Dec 2 & $\begin{array}{l}\text { See also analysis by Kohno et al. (2002), } \\
\text { Nakajima et al. (2003) }\end{array}$ \\
\hline GGD 12-15 & $061050.00,-061200.00$ & 12392 & 67.3 & 2011 Dec 23 & $\begin{array}{l}\text { X-ray catalog not yet in the literature; X-ray } \\
\text { properties to be discussed in detail in the } \\
\text { corresponding YSOVAR cluster paper }\end{array}$ \\
\hline NGC 2264 & $\cdots$ & $\cdots$ & $\cdots$ & $\cdots$ & $\begin{array}{l}\text { Reanalysis beyond the scope of this paper; } \\
\text { values taken from Flaccomio et al. (2006) } \\
\text { and Ramirez et al. (2004a) }\end{array}$ \\
\hline L1688 & $162717.18,-243439.00$ & 635 & 100.7 & 2001 May 16 & See also analysis by Imanishi et al. (2001) \\
\hline Serpens Main & $182950.00,+011530.00$ & 4479 & 88.5 & 2005 Jun 28 & $\begin{array}{l}\text { See also analysis by Winston et al. (2007), } \\
\text { Giardino et al. (2007) }\end{array}$ \\
\hline Serpens South & $183003.00,-020158.20$ & 11013 & 99.5 & 2011 Jun 10 & $\begin{array}{l}\text { X-ray catalog not yet in the literature; X-ray } \\
\text { properties to be discussed in detail in the } \\
\text { corresponding YSOVAR cluster paper }\end{array}$ \\
\hline IRAS $20050+2720$ & $200713.60,+272848.80$ & 6438 & 22.6 & 2006 Dec 10 & See also analysis by Günther et al. (2012) \\
\hline IRAS $20050+2720$ & $200713.60,+272848.80$ & 7254 & 20.9 & 2007 Jun 7 & (As above) \\
\hline IRAS $20050+2720$ & $200713.60,+272848.80$ & 8492 & 50.5 & 2007 Jan 29 & (As above) \\
\hline IC1396A & $213650.30,+573024.00$ & 10990 & 29.7 & 2010 Jun 9 & See also analysis by in Getman et al. (2012) \\
\hline IC1396A & $213650.30,+573024.00$ & 11807 & 29.8 & 2010 Mar 31 & (As above) \\
\hline Ceph C & $230551.00,+623055.00$ & 10934 & 44 & 2010 Sep 21 & $\begin{array}{l}\text { X-ray catalog not yet in the literature; X-ray } \\
\text { properties to be discussed in detail in the } \\
\text { corresponding YSOVAR cluster paper }\end{array}$ \\
\hline
\end{tabular}

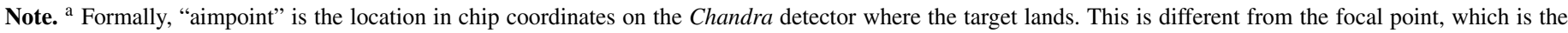
location of the sharpest (narrowest) point-spread function. In practice, this can be thought of as the target of the observation.

Table 6

X-ray Source Detection Characteristics

\begin{tabular}{|c|c|c|c|c|c|c|}
\hline Cluster & No. $>2 \sigma^{\mathrm{a}}$ & No. Cryo Match ${ }^{b}$ & Detections in Literature & $\operatorname{Min}\left(\log L_{x}\right)^{\mathrm{c}}$ & $\operatorname{Max}\left(\log L_{x}\right)^{\mathrm{c}}$ & $\operatorname{Med}\left(\log L_{x}\right)^{\mathrm{c}}$ \\
\hline AFGL 490 & $\ldots$ & $\ldots$ & (No X-ray data) & $\ldots$ & $\ldots$ & $\ldots$ \\
\hline NGC 1333 & 192 & 119 & 109 (Getman et al. 2002), 193 (Winston et al. 2010) & 27.94 & 30.74 & 29.12 \\
\hline Orion & $\cdots$ & $\cdots$ & Using lit.; see text & 27.58 & 32.62 & 29.72 \\
\hline Mon R2 & 492 & 167 & $154(5 \sigma ;$ Kohno et al. 2002) & 29.58 & 31.16 & 30.16 \\
\hline GGD 12-15 & 229 & 172 & $\ldots$ & 29.66 & 31.77 & 30.33 \\
\hline NGC 2264 & $\ldots$ & $\ldots$ & Using lit.; see text & 28.45 & 31.29 & 29.95 \\
\hline L1688 & 315 & 69 & 11 (Imanishi et al. 2001) & 27.48 & 31.30 & 29.51 \\
\hline Serpens Main & 204 & 161 & 85 (Giardino et al. 2007) & 29.13 & 31.71 & 29.97 \\
\hline Serpens South & 294 & 82 & $\cdots$ & 29.00 & 31.27 & 29.82 \\
\hline IRAS $20050+2720$ & 348 & 239 & 239 (Günther et al. 2012) & 29.28 & 30.88 & 30.12 \\
\hline IC 1396A & 185 & 129 & $415^{\mathrm{d}}$ (Getman et al. 2012) & 29.14 & 32.26 & 30.34 \\
\hline Ceph C & 200 & 97 & $\ldots$ & 29.44 & 30.95 & 30.17 \\
\hline
\end{tabular}

Notes.

${ }^{a}$ Number X-ray sources detected via our reprocessing at $>2 \sigma$.

b Number of our X-ray sources matched to sources in the IRAC cryogenic-era catalog.

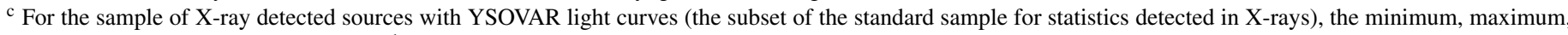
and median $\log L_{x}$, where $L_{x}$ is in erg s${ }^{-1}$.

d The detections used in Getman et al. (2012) do not require IR matches, and they go well below our typical significance cut off.

constancy. It assigns an index to each light curve-the higher the value of the index, the greater the variability. Index values greater than 7 indicate $>99 \%$ variability probability. Values of the GL-vary index $>9$ usually indicate flares. GL-vary is not reliable below about 30 raw counts, the same limit we used for performing spectral fits. The value of this index will be provided where relevant on a source-by-source basis in the individual cluster papers.

There are five broad classes of X-ray sources in the field.

1. First, background active galactic nuclei(AGNs) - these will be numerous; up to 50 of these per $16^{\prime} \times 16^{\prime}$ Chandra field 
are expected, depending on the depth of exposure (Getman et al. 2006). However, they tend to be faint in both X-rays and mid-IR, or are not matched in the IRAC bands at all.

2. Second, background starburst galaxies-while much less common than AGNs, they are brighter than AGNs, and have colors similar to Class II YSOs. They tend to be fainter in the IR than typical Class II YSOs, and may be tentatively identified via the faintness of the IR counterpart.

3. Third, compact objects such as white dwarfs-these tend to be very faint and usually undetected in the IRAC bands.

4. Fourth, YSOs - those with disks have already been identified via their IR excesses. We identify the probable diskfree objects that are detected in X-rays by their star-like IR colors and magnitudes consistent with membership. In addition to matching an IRAC source, the star-like colors are required to ensure that any newly revealed sources are probable Class III objects and not distant starburst galaxies.

5. Fifth and finally, active late-type field stars-both foreground and background stars can appear with X-ray fluxes comparable to our targets. Based on a study of IC 1396, Getman et al. (2006) estimate that there could be $\lesssim 10$ of these per $16^{\prime} \times 16^{\prime}$ Chandra field. Since the contaminants are a mixture of foreground and background objects of comparable fluxes to our targets (with less foreground and more background contamination likely for closer clusters), without optical spectra, they are very hard to discern from Class III objects. It is necessary, then, that these remain in our sample of candidate members selected via X-rays and represent a source of contamination.

We note that Getman et al. (2012) estimate (again for IC 1396 , a region in the Galactic plane) a $22 \%$ probability that any X-ray source with any IRAC counterpart is not a member of the cluster. That rate drops to about $10 \%$ in IC 1396 if one eliminates sources without 2MASS $J H K_{s}$ detections. Because the contamination rate is affected by absorption, exposure time, and the depth to which one extracts sources, it may be different for the other clusters.

We can improve our inventory of YSOs in these clusters by identifying objects with X-ray detections, IRAC counterparts, and SEDs that are consistent with those of stars. We have adopted this YSO selection mechanism as the other main component of the primary sample definitions; see Section 3. Note that we define SEDs consistent with stars to be those with a fitted SED Class III (see Appendix B), but that there is room to create an augmented membership list (Section 3.3) to include objects that have an X-ray detection, an IRAC counterpart, were not identified as a YSO from the IR alone, but that have an SED consistent with a YSO. With regard to foreground or background stellar contamination, because any appropriate brightness cutoff is a function of cluster distance (and $A_{V}$ ), we defer any detailed exclusion of likely foreground or background stars from the member sample to the individual cluster papers.

\section{SAMPLE DEFINITIONS}

For most of our clusters, there are no well-established membership lists; the exceptions are Orion and NGC 2264. Moreover, the membership lists in the literature use a wide variety of wavelengths and survey depths to identify members, and the spectroscopic follow-up of candidate members is uneven. If we decided to depend on the robust member identifications only from the literature, our sample would be greatly reduced for most clusters and highly biased. Certainly, our variability survey will identify new candidate members based on the light curve properties. To attempt to make fair comparisons between clusters, we need to define a set of (candidate) members in the same or at least consistent ways between clusters.

As discussed above, even within our survey, different clusters may have different amounts of monitoring data beyond the "fast cadence" data. Thus, for making comparisons between clusters, we need to define a standard set of data that are used for calculating statistics and identifying variables.

Therefore, we define a "standard YSOVAR sample," which is the sample that is (primarily) discussed in this paper and which forms the common core of the papers planned for each cluster. Each cluster may have an additional "augmented sample" as well, to take into account additional member identifications from the literature or our own data where possible and necessary. We now discuss the definitions of these samples.

\subsection{Standard Set of Members}

Each cluster has an IR-selected sample of member candidates defined by the Gutermuth et al. (2008b, 2010) and G09 selection algorithm, run on the cryo-era catalogs created anew as per the methodology above (Section 2.7). A detailed description of the YSO candidate color selection algorithm can be found in Appendix A of G09. A sample of YSOs selected this way is thought to be a statistically well-defined sample (see statistical discussions in Gutermuth et al. 2008b and G09), comprised almost entirely of members, though some contamination from background galaxies or asymptotic giant branch stars is always possible. Very few of these IR-selected members have spectroscopic follow-up (or spectra in the literature pre-dating the Spitzer observations), and, as such, many should technically be thought of as YSO candidates, though we include them all in the set of members.

All clusters except AFGL 490 have X-ray data, and thus also an X-ray-selected sample of YSOs. (As with the IR-selected sample, few of these have spectra, so technically they are YSO candidates.) Since we should have identified most of the YSOs with disks (at least disks detectable at 3.6-24 $\mu \mathrm{m}$ ) in the IR selection process, we use the X-ray data to identify additional young stars without disks (e.g., Class IIIs). Thus, we add to the set of IR-selected members the X-ray-selected sample defined by the algorithm described above in Section 2.10, which can be summarized as objects having an X-ray detection above a $2 \sigma$ significance threshold, having a match to an IRAC object in the cryo-era Spitzer catalog, and having an SED shape consistent with it being a disk-free YSO or a star (e.g., Class III; see Appendix B.2). This sample is specifically designed to find and add to our set of members those members without disks. However, it should be noted that (1) the Galactic contamination rate is likely to be higher in this X-ray-selected sample than in the IR-selected sample; and (2) specifically because of the contamination rates, members with disks are identified as members from the IR excess, not the X-ray flux, though, of course, members with disks can also have measured X-ray fluxes in our database.

We have thus defined our "standard set of members" to be the union of all IR-selected members with disks and $\mathrm{X}$-ray-selected members without disks. There are provisions for adding additional objects; see Section 3.3 below. Note that this definition can be applied independently of whether or not there is a light curve, but of course in the context of this discussion of YSOVAR data we require a light curve. Note also 
that the IR selection requires four bands of IRAC, and thus both very faint and very bright previously identified members may be omitted from the standard set; objects such as these known to be members via some other approach in the literature may be added in the augmented sample (Section 3.3). Finally, note that because the data that go into our selection of cluster members are of various depths, and because the clusters are at a variety of distances, the effective mass limit reached by each set of standard members varies from cluster to cluster.

\subsection{Standard Set for Statistics}

All of the original YSOVAR light curves were obtained with very similar HDR mapping observations (see Section 2.2) in a fast cadence (see Section 2.4), though the length varies, and some clusters have additional slow cadence observations and/or staring observations. The time sampling and total length of light curves obtained within a single cluster field can also vary as the FOV changes with time (Section 2.3). We have attempted to remove all instrumental effects from the input data, and only retained photometry where there were valid measurements on at least three BCD frames (Section 2.5).

We now define the "standard set of data for statistics" as follows. Since the fast cadence is the most common (and most similar) among the YSOVAR clusters, we used only these mapping fast cadence data, for those light curves that have at least five viable epochs (with each epoch obtained from at least three BCDs per epoch that are not obviously compromised by instrumental effects or cosmic rays), to calculate statistical quantities such as mean, median, etc., as well as Stetson index and $\chi^{2}$ (discussed below; see Section 5). We have defined statistical values calculated on the fast cadence data as the "standard set of statistical values" for each cluster and employ them to identify variables and compare values across clusters. Finally, for stars fainter than [3.6]-[4.5] 16, noise tends to dominate the light curves (Sections 4.2 and 5). We have retained these faint sources, but objects this faint are considered individually where relevant.

Note that the standard set for statistics is thus defined as all light curves with at least five points, just in the fast cadence. This is independent of whether or not the target is identified as a member.

Elsewhere in this paper, we refer to "all objects with a light curve"-this means anything with a light curve in the standard set for statistics. (Essentially no YSOVAR-classic sources have only points outside of the fast cadence.)

When available, additional epochs of data can be included for additional calculations on a per-cluster basis in the corresponding papers, and will clearly be indicated as such where relevant.

\subsection{Augmented Sample of Members}

We identified members above using IR and X-ray data, which implicitly relies on the shape of the SED between 2 and $24 \mu \mathrm{m}$. That sample is still the best set of members that we will use to compare across clusters, because that set of members is defined as similarly as possible across all clusters. However, additional young objects may be identified in the literature, and additional members may be suggested based on our own data. The "augmented sample of members" is where these additional likely members can be included.

Some clusters (e.g., NGC 1333, L1688) have considerable literature discussion of members, and others (e.g., AFGL 490,
Mon R2) have far less. We therefore cannot rely exclusively on the literature to select members, but neither should we ignore members identified in the literature and not selected above. Thus, each cluster paper may include in the augmented sample the literature-identified sources that are not already found using our IR or X-ray methods above.

We can use our own data to identify new cluster members. While only $1 \%-2 \%$ of the field population may be variable, YSO variability is the rule, not the exception. It is therefore possible to identify new cluster members from light curve properties alone; one could identify all variables as new members, or one could take only those with certain properties such as amplitude above a threshold. We could identify cluster members from either the standard set for statistics (the fast cadence), or, for those clusters with longer cadences, from those additional data.

The set of statistically selected variables (see Section 5) are those with Stetson index greater than 0.9, and/or with $\chi^{2}$ greater than 5, and/or with a significant period, calculated over only the standard set for statistics (the fast cadence). Often, these variables should also be identified as cluster members, but these individual objects will be discussed on a per-cluster basis (because, for example, they can be background eclipsing binaries; Morales-Calderón et al. 2012). Variables identified using data beyond the standard set for statistics (data beyond the fast cadence data) will also be included on a per-cluster basis, and will be discussed in the cluster papers. Those newly identified cluster members may also be included in the augmented sample of members.

While variability-identified objects will make an important contribution to our understanding of the complete membership of each cluster, they should not be used in calculations of, e.g., variability fractions; that sample should be selected on the basis of a parameter distinct from variability, such as disk excess or $\mathrm{X}$-ray emission. This is why the new candidate members we identify from our data are included in the augmented sample of members and not in the standard set of members.

This augmented set of members is only used (where it is clearly identified) in the individual cluster papers, not in the remainder of this paper.

\section{ENSEMBLE ANALYSIS}

In this section, we present an analysis of the entire set of data we used for our clusters, independent of variability, which is discussed in Section 5.

\subsection{Cluster Parameterization}

In order to compare results among clusters, it is useful to be able to place clusters in some sort of relative order that could, in the most useful (though hypothetical) case, be tied to age. There are various ways of parameterizing the evolutionary state of these clusters, and we considered several, all aimed at capturing the relative numbers of sources in various SED class bins (see Appendix B for a brief definition of SED classes and our placement of sources therein). Such ratios in some sense capture the relative "degree of embeddedness" of sources in these regions, perhaps with an ultimate (though undefined here) link to cluster ages. Formally, we are binning by SED slope, so the parameterizations are, strictly speaking, relative fractions of sources with a given SED slope, indicative of the amount of circumstellar material, e.g., how self-embedded a given source may be. We interpret clusters with more sources with large 
SED slopes to, on average, contain more sources that are more embedded.

G09 chose to use the ratio of Class II to Class I sources. These ratios were all obtained internally consistently, e.g., sources selected and categorized according to the same series of IR color cuts and data reduction. Because G09 was working with Spitzer data over a relatively large region for each cluster, this Class II to Class I ratio could be calculated for the whole region and for subsections of the region. The Class II to Class I ratios as calculated for the cores of these clusters (e.g., Table 6 in G09) appear in our Table 1 and in the discussion in Section 2.1.2.

In YSOVAR, we have clusters not included in G09, and moreover, even for the clusters included in G09, we generally have light curves for only a small subset of the sources G09 considered because we observed a smaller region. To calculate a Class II to Class I ratio for the portion of each cluster sampled by the YSOVAR monitoring, we performed the same calculation for objects in the standard set for statistics (e.g., having YSOVAR light curves) by reducing the cryogenic data in the same way and performing the same series of G09 color cuts and classification; see Section 2.7. Then, we recalculated the ratio of Class II to Class I sources specific to the YSOVAR data using the classes assigned via the G09 algorithm only for those sources with light curves. Those Class II to Class I ratios also appear in Table 1 and in the discussion in Section 2.1.2. The values are provided in the present work in part as a link to the G09 analysis; note that they are calculated in the same way as G09, e.g., with the IR-selected sources alone, not on the standard set of members per se.

The G09 parameterization is based only on IR-selected sources. For most of our clusters, we have X-ray data as well (see Section 2.10), so we at least have some information on the Class III population. It is, however, true that we do not always have complete Chandra coverage of our fields (further discussion of coverage appears in Section 2.10 and Figures 1-13), and even for clusters where we have Chandra coverage, the Chandra sensitivity is a strong function of location on the array. Nonetheless, we would like to include the information we have, and simply using the Class II to Class I ratio does not incorporate information about the Class III population.

We explored several alternative parameterizations of the relative fractions of embedded sources, all of which involved various ratios of classes (or groups of classes) to the total or other classes (or groups of classes). Histograms of the relative fractions of the SED classes for the standard set of members (Section 3.1) for objects with light curves in the standard set for statistics (Section 3.2) in each cluster appear in Figure 17. AFGL 490 can be seen to be deficient in a complete sample of Class III objects because it has no X-ray data; the Class III objects identified here have small IR excesses (or sufficient reddening at $2 \mu \mathrm{m}$ ) and thus SED slopes consistent with Class III. By many metrics, Serpens South has the highest fraction of sources with positive SED slopes, which we take to be most embedded sources. Similarly, both Orion and IC 1396A have the highest fraction of sources with negative SED slopes, which we take to be less embedded sources.

For further analysis here, we have settled on the fraction of Class I sources to the total number of members, for objects with light curves (objects in the standard set for statistics). These ratios are also included in Table 1. They include (as part of the total number of members) objects selected via X-rays. However, there are still the fundamental, systematic uncertainties inherent in the classification approach, in the selection of members

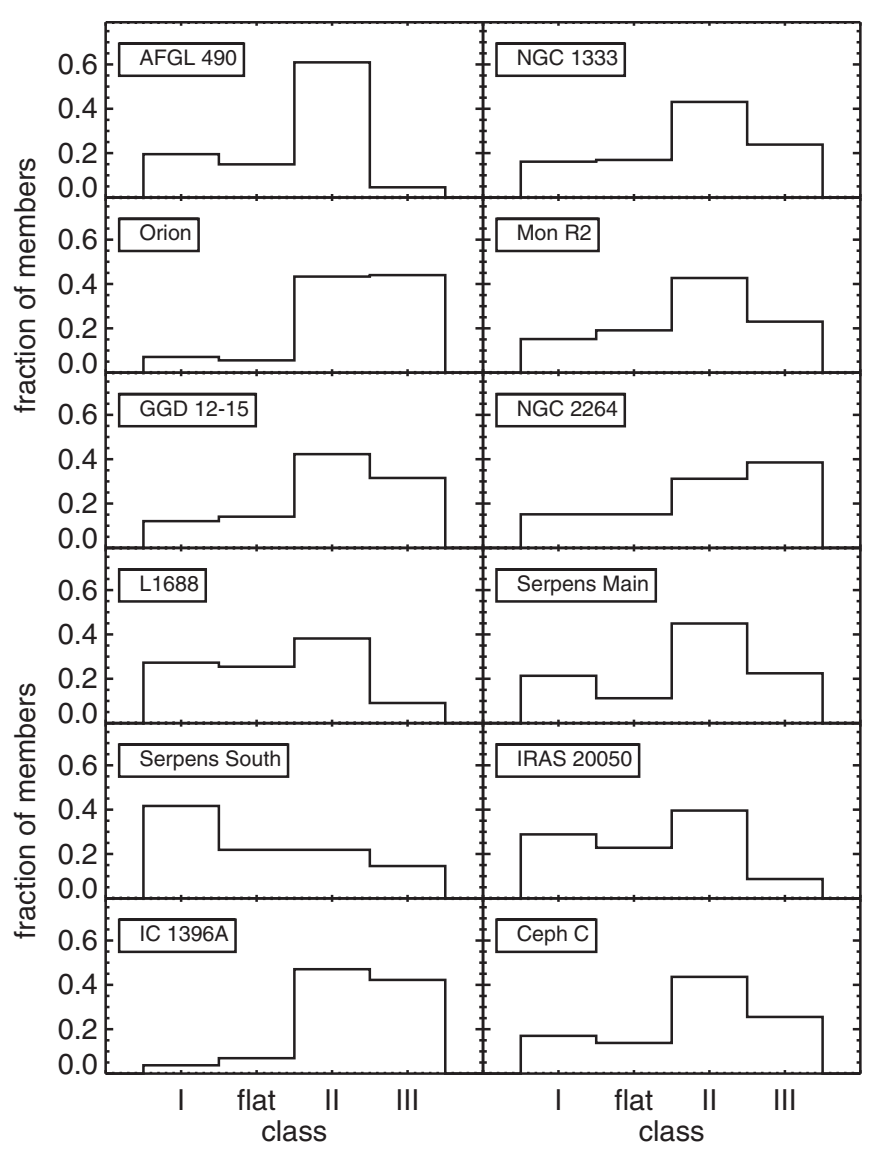

Figure 17. Histograms of the relative fractions of fitted SED classes (classes derived from SED fits as discussed in Appendix B.2) for the standard set of members with light curves. Clusters appear in R.A. order. By nearly any ratio of classes used as a parameterization, IC 1396A and Orion have the highest fraction of sources with negative SED slopes (taken to be less embedded sources), and Serpens South has the highest fraction of sources with positive SED slopes (taken to be most embedded sources). AFGL 490 is noticeably incomplete in the Class III bin; there are no X-ray observations available for it, and the Class III objects in that bin have small IR excesses and SED slopes consistent with Class III (see Appendix B for a description of classes and class selection).

without spectroscopic follow-up, in the completeness of the surveys involved (in both area and depth), and the requirement that there be a light curve (e.g., bright enough in the IRAC channels) as well as in the relative paucity of Class I objects overall. Additionally, for AFGL 490, there are no X-rays to be used, so the ratio is calculated with solely the IR-identified members. However, the Class III objects appear only in the denominator, combined with all the other classes. We also note that our inventory of Class I sources must be incomplete, since we lack the long wavelength coverage that would be needed to find the most embedded sources (e.g., Stutz et al. 2013), but such extremely embedded sources will also not have a YSOVAR light curve.

This parameterization using the Class I/total ratio should be related to the G09 Class II/Class I ratio determined for the objects having light curves. Figure 18 plots these parameterizations against each other. The error bars are derived assuming uncorrelated Poisson statistics, because it is difficult to quantify the additional systematic errors described above. The best-fit slope of the line fit to this relation (taking into account errors in both directions on each point) is $-0.024 \pm 0.005$. The correlation coefficient, Pearson's $r$, calculated for these two parameters is -0.87 , and a probability that the parameterizations are not 


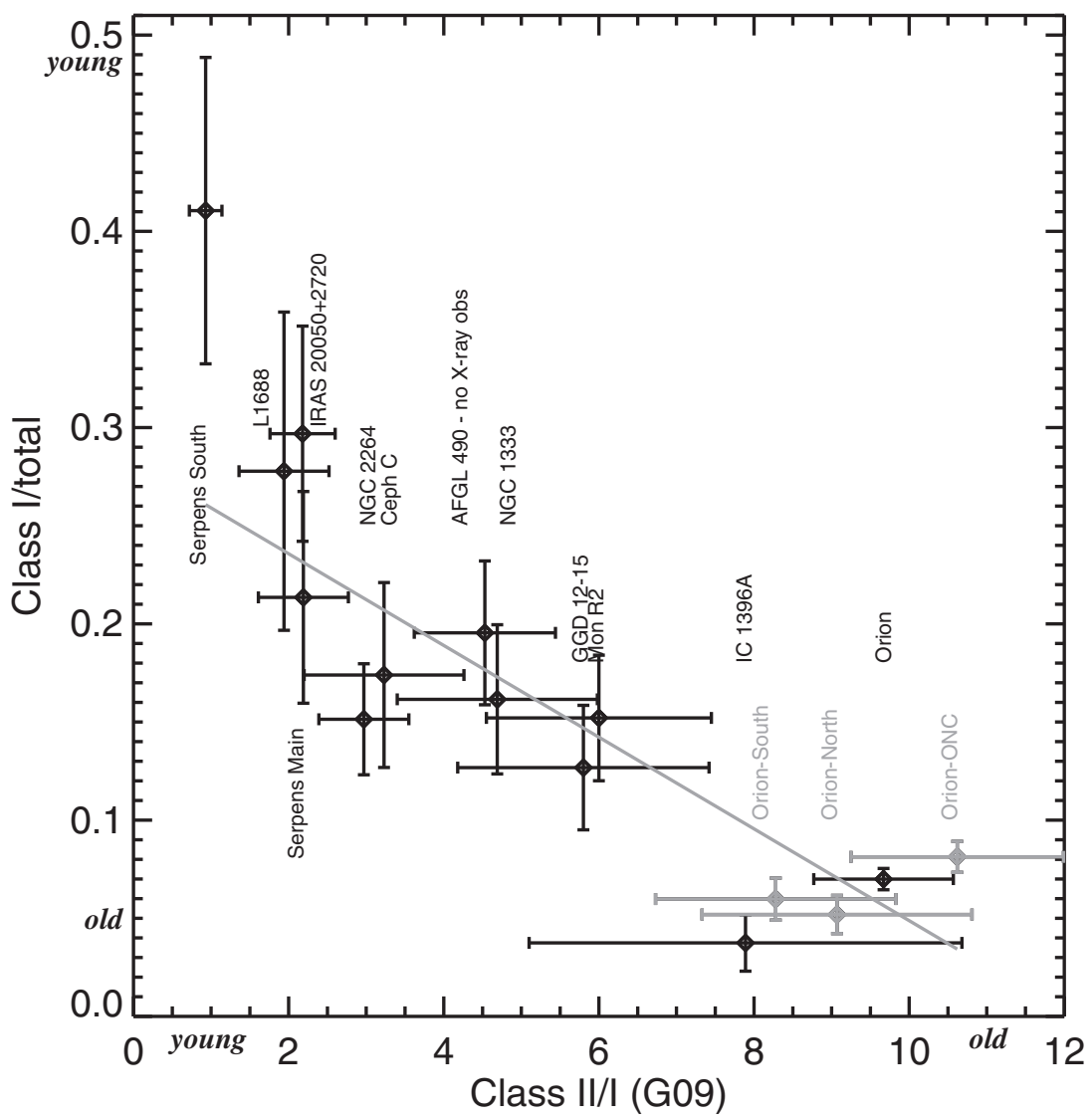

Figure 18. Comparison of the Class II/I parameterization from G09 and the Class I/total parameterization used here. All values are calculated only for objects with light curves (the standard set for statistics; Section 3.2); the G09 values use only the IR-selected members from the G09 approach, and the Class I/total values use the standard set of members (Section 3.1). The error bars are derived assuming uncorrelated Poisson statistics. Cluster labels appear at the Class II/Class I location corresponding to the cluster. The gray line is the best-fit line, using errors in both directions on each point, and has a slope of $-0.024 \pm 0.005$. Pearson's correlation coefficient $(r)$, calculated for these points is -0.86 ; the calculated probability that the parameterizations are not correlated is only $0.04 \%$. We take these values to be correlated. The additional gray points are subregions of Orion, used here to show the scatter inherent in the large Orion map (see the text). Notional "young" and "old" annotations on the axes describe approximate relative ages that may quantitatively correspond to large or small values of these parameterizations.

correlated of only $0.04 \%$. We assume based on the statistics and our underlying physical intuition that these values are indeed correlated.

Several individual points in Figure 18 merit additional discussion. Despite having no X-ray data, AFGL 490 is consistent with the trend shown in Figure 18. (If one assumes that there might be about as many Class IIIs as Class IIs in this region, then the point could move down to about $0.1-0.15$, which would still be broadly consistent with the trend.) The NGC 2264 point is below the trend. The X-ray data obtained for NGC 2264 is deeper than the X-ray data for the other clusters, except for the central Orion region. This results in more of the fainter sources being included in the total number of YSOs, and pushes the Class I/total ratio toward lower numbers, as seen. For both parameterizations, Serpens South is selected as the cluster with the most embedded sources, as expected from Figure 17. It is well above the fitted line in Figure 18; perhaps an exponential decay rather than a simple line would be a better fit to use, but, in the absence of additional very embedded clusters to constrain the most embedded end of the distribution (or, indeed, spectroscopic vetting of the members and the reduction of other such uncertainties), a line is the simplest fit to use. IC 1396A, based on Figure 17, should be one of the clusters with the fewest embedded sources. It is identified as the least embedded using the Class I/total ratio; there is a large uncertainty on the Class II/ Class I ratio, and it is consistent with being the least embedded within $1 \sigma$. The Class II/Class I ratio formally identifies Orion as the least embedded. However, aside from AFGL 490 where there are no X-ray data, Orion is the cluster with the poorest match between the YSOVAR-monitored region and the existing $\mathrm{X}$-ray data coverage. Orion has a very deep X-ray pointing, but only in the central ONC region (Feigelson et al. 2005; Getman et al. 2005). There are two shallower pointings that contribute Xray data (see Section 2.10 and Figure 3), but the region of sky in Orion for which we have IR light curves has the least fractional coverage in X-rays of all our clusters (aside from AFGL 490). To investigate the degree to which the uneven X-ray coverage affects the placement of Orion in this diagram, Figure 18 also includes points for Orion when broken into "North" (declination $>$ $-05: 05: 25)$, "South" (declination $<-05: 33: 15^{\circ}$ ), and "ONC" (between those two limits) fields. There is scatter, clearly, in these points, but when those points are used instead of the single Orion point, the fit is functionally indistinguishable from that using the single Orion point.

We use the Class I/total parameterization in subsequent discussions in this paper, most notably Section 6.

\subsection{Brightness Distribution at J, [3.6], and [4.5]}

Figures 19 and 20 show the distribution of $J$, [3.6], and [4.5], in units of magnitude and the percent of the sample, for the standard set for statistics (all objects with at least five points in the YSOVAR fast-cadence light curve, including cluster members and field objects). The total number of objects 


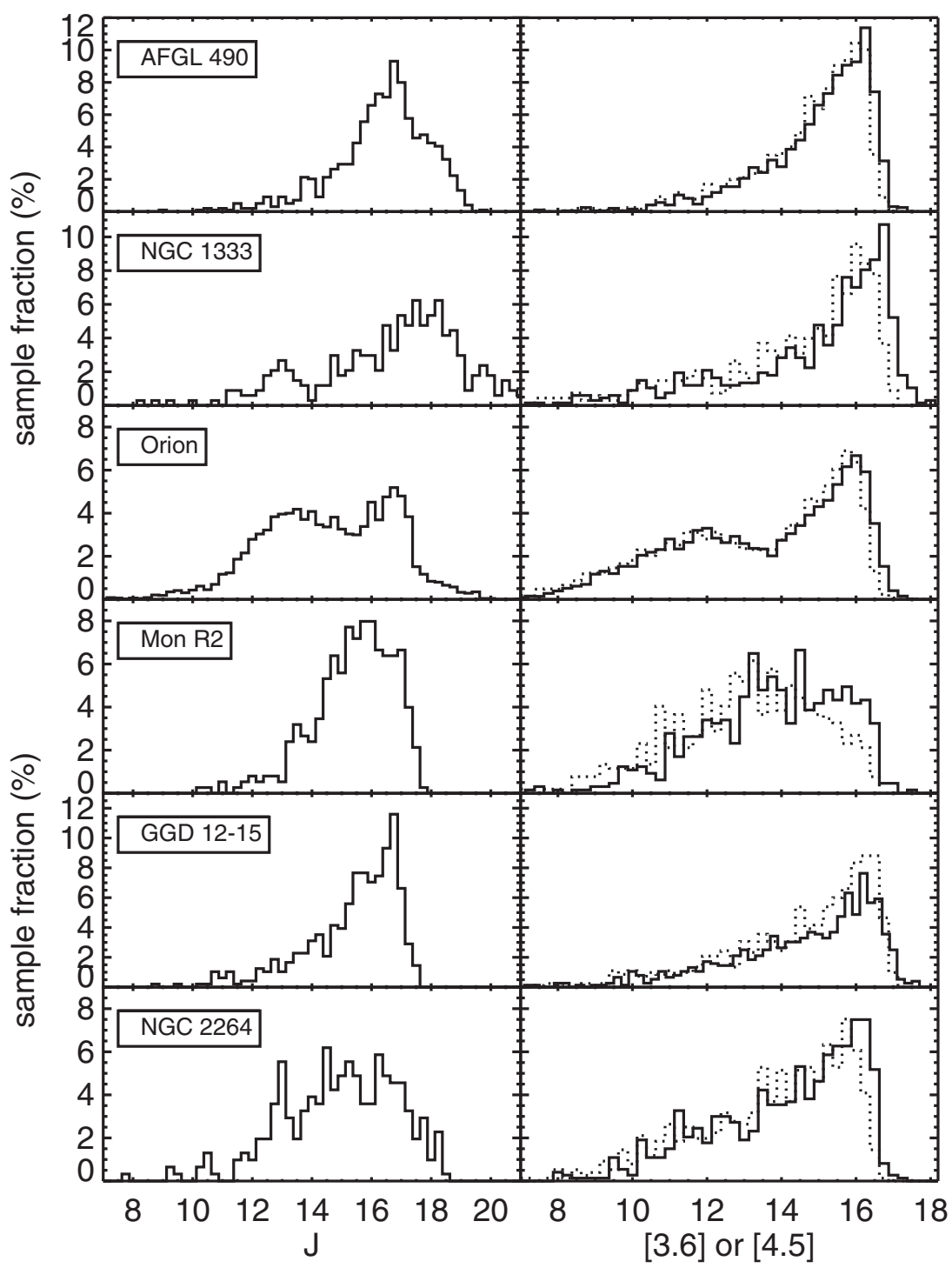

Figure 19. Distribution of $J$ magnitudes (left) and IRAC magnitudes (right; [3.6] in the solid line and [4.5] in the dotted line), for the standard set for statistics (all objects with light curves), in units of fraction of sample (in \%) for each cluster. This figure shows AFGL 490, NGC 1333, Orion, Mon R2, GGD 12-15, and NGC 2264. See the text for discussion. As a result of these plots, we are cautious about objects fainter than [3.6]-[4.5] 16, both because they have low signal-to-noise in our monitoring data and because they are likely dominated by non-members.

portrayed in the figures ranges from $\sim 100(\mathrm{~J}: \mathrm{L} 1688$ or Serpens South) to $\sim 7200$ ([3.6] and [4.5]: Orion). The $J$ data are generally from 2MASS; notably, NGC 1333 is deeper than the other clusters in $J$ because additional data from the $6 \times$ 2MASS survey have been included (Section 2.9). Generally, more objects are available at [3.6] or [4.5] than at $J$, largely due to the greater effective depth of Spitzer. In several cases (most notably L1688, Serpens South, and NGC 2264), a relatively small fraction of the objects with Spitzer light curves have $J$ counterparts, since these clusters are, on average, generally more embedded than the others. For most of the clusters, for most of the objects, $J H K_{s}$ data are not available for objects with [3.6] or [4.5] fainter than about 15 th mag.

The Orion maps extend out beyond the edges of the cluster, and include a higher proportion of field stars and other contaminants than do the other smaller-field clusters. This can be seen in the structure in the Orion [3.6] and [4.5] histogram, which is double-peaked; the brighter peak is likely dominated by the cluster members, and the fainter peak is likely dominated by contaminants. For similar reasons, if the UKIDSS data
(Section 2.9) are included, the Serpens Main $J$ histogram extends to $J \sim 20$ and is also double-peaked, but the L1688 $J$ histogram is not so obviously double-peaked, likely due to the higher obscuration levels of the background population.

Mon R2 seems to be different from the other clusters in that the fainter end of the [3.6] and [4.5] histograms are considerably flatter. This is most likely a symptom of the difficulty of obtaining Spitzer light curves for faint sources in the presence of high and spatially variable background; there are some very IR-bright sources in the IRAC FOV (see Figure 4), and the scattered light is substantial, coupled with intrinsically bright outflows and PAH features. No UKIDSS $J$ mag in this region were in the public archive at the time we checked (in 2013 September).

As can be seen from the turnover at about 16th mag in the [3.6] and [4.5] histograms in Figures 19 and 20, our data do not extend much fainter than [3.6]-[4.5] 16 mag. By inspection, all of these faint objects appear to be legitimate point sources on the images. For stars fainter than this, noise tends to dominate the light curves (see Section 5). It is hard to completely reject 


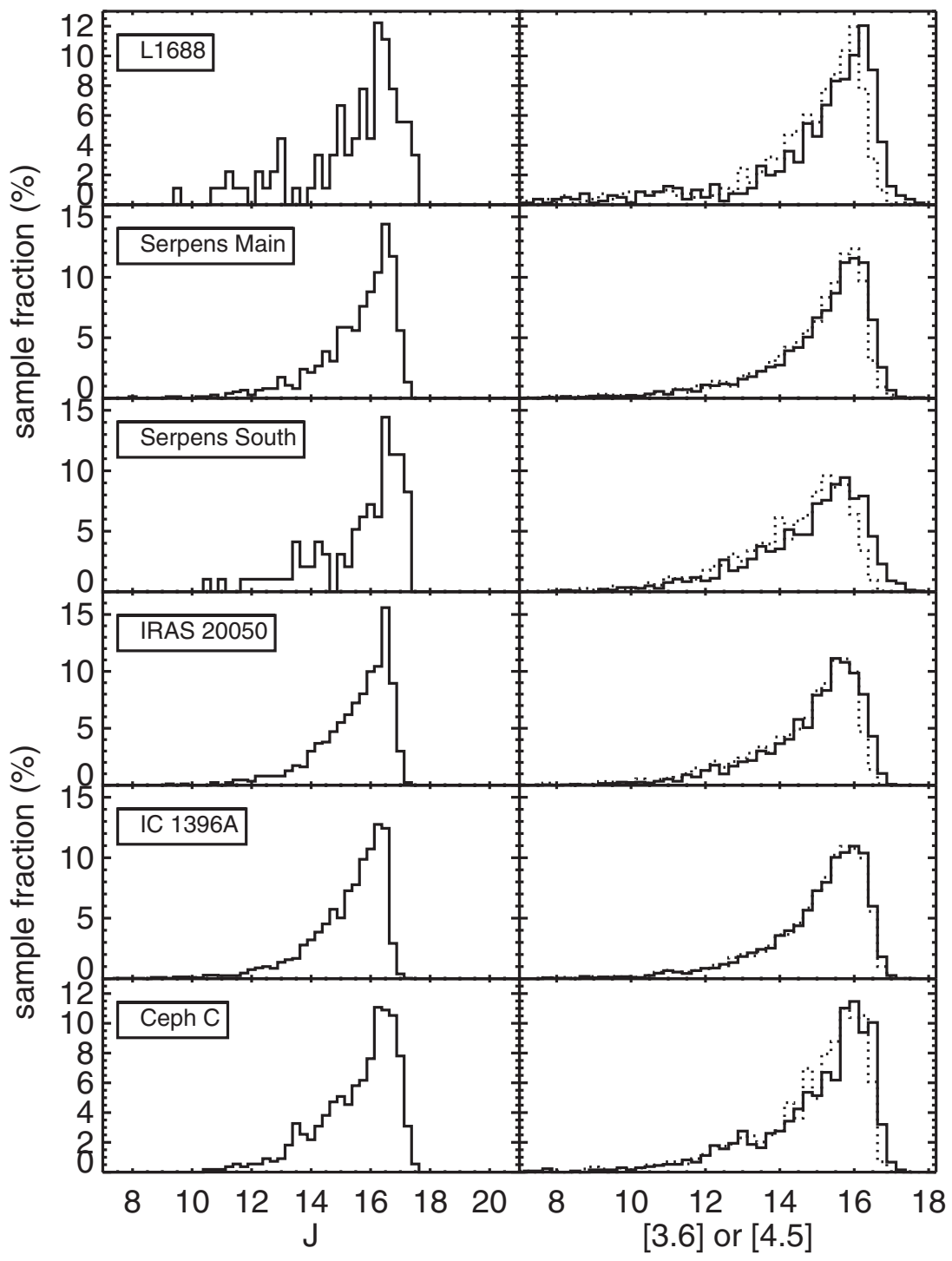

Figure 20. Same as for Figure 19, but for L1688, Serpens Main, Serpens South, IRAS 20050+2720, IC 1396A, and Ceph C.

these fainter sources - we could exclude those whose cryogenicera [3.6] > 16, though the YSOVAR epochs could vary above and below that boundary; or we could discard those where the mean during the YSOVAR campaign is $>16$, but there are some objects for which the mean [3.6] $<16$ but the mean [4.5] $>16$. As noted in Section 3, we have retained these faint sources, but, in general, the large uncertainties associated with their Spitzer photometry preclude strong statements about their variability; objects this faint are considered individually where relevant.

Similar histograms for only the standard set of members (identified through IR excess and X-ray emission; see Section 3) are considerably less populated, and brighter; the peaks are around [3.6]-[4.5] $\sim 12 \mathrm{mag}$. This is consistent with the location of the brighter peak in Orion, and several of the tails of the distributions seen in Figures 19 and 20.

\subsection{X-Ray Brightness Distribution}

Figure 21 contains histograms of the log of the X-ray luminosities $\left(\log L_{x}\right.$, where $L_{x}$ is in erg s$\left.{ }^{-1}\right)$ for those objects with light curves (standard set for statistics) and bright enough in flux $\left(F_{x}\right)$ to have a calculated $L_{x}$ (Section 2.10). The Orion and NGC 2264 histograms reach fainter values in $F_{x}$ than those of the other clusters because those integrations were considerably deeper. Moreover, essentially the entire NGC 2264 field considered here has X-ray data, whereas the fractional $\mathrm{X}$-ray coverage of the Orion field is relatively low compared to NGC 2264 or the other clusters here (aside from AFGL 490, where there is no X-ray data). Because this figure has incorporated distance (distances are listed in Table 1) to the clusters in the calculation of $L_{x}$, the two closest clusters (NGC 1333 and L1688) have histograms reaching the faintest $L_{x}$

\section{IDENTIFYING VARIABLES}

There are many ways discussed in the literature of identifying variables in time series data. We tested several methods, and settled on three primary ones, which we now discuss in separate subsections. Recall that we are calculating statistics for the standard statistical sample, e.g., on only the fast cadence data, for those objects with at least five viable data points in the light curve (Section 3).

\subsection{Stetson Index}

The first way we identify variables is the Stetson index (Stetson 1996), which quantifies correlation of variability in 


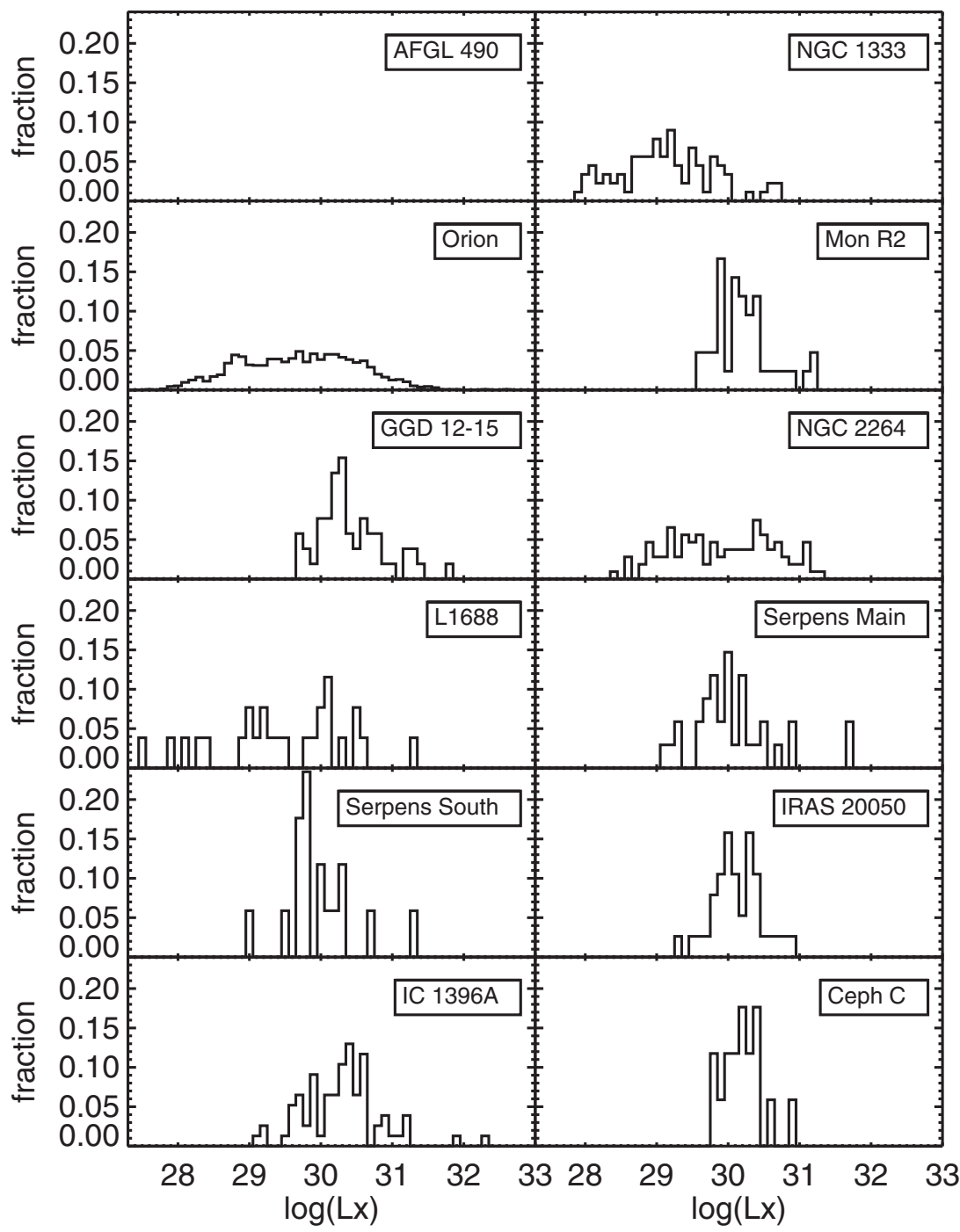

Figure 21. Distribution of $\log \left(L_{x}\right)$ values (in erg s ${ }^{-1}$ ) for the objects in the standard set for statistics (with $L_{x}$ detections) in our clusters. Note that there are no data available for AFGL 490, and we use literature data for NGC 2264 and Orion; see the text. L1688 and NGC 1333 are the closest clusters, and so relatively faint $L_{x}$ measurements are obtained with even relatively shallow observations. NGC 2264 and Orion have the deepest integrations and therefore also include relatively faint $L_{x}$.

two (or more) bands. The Stetson variability index is computed for each object as:

$$
S=\frac{\sum_{i=1}^{N} g_{i} \times \operatorname{sgn}\left(P_{i}\right) \times \sqrt{\left|P_{i}\right|}}{\sum_{i=1}^{N} g_{i}},
$$

where $N$ is the number of pairs of observations for a star taken at the same time ${ }^{33} P_{i}=\delta_{j(i)} \delta_{k(i)}$ is the product of the normalized residuals of two observations, and $g_{i}$ is the weight assigned to each normalized residual. In our case, the weights are all equal to one. The normalized residual $(\delta)$ for a given band is computed as:

$$
\delta_{i}=\sqrt{\frac{N}{N-1}} \frac{\operatorname{mag}_{i}-\overline{\operatorname{mag}}}{\sigma_{i}},
$$

where $N$ is the number of measurements used to determine the mean magnitude and $\sigma_{i}$ is the photometric uncertainty. Objects

\footnotetext{
33 The I1 and I2 maps are taken of any given source (providing it falls within the region with two-band coverage) typically within $\leqslant 12$ minutes of each other. For these observations taken on a few-epochs-per-day cadence, we are not sensitive to timescales of minutes, and the data points are effectively simultaneous.
}

with larger values of the Stetson index are typically taken to be variable. Since errors are included in the calculation, light curves that are just noisy are not identified as variable. Objects with variability in different bands that is not correlated will not be identified via this method; physically, we expect most YSOs to have similar variations in the two IRAC channels since it is difficult to imagine processes that would make one IRAC channel vary without the other. However, this method will not find variables in cases where one IRAC channel is compromised, e.g., due to instrumental effects, and the other is not.

If there is correlated noise between the two channels used for the Stetson index, especially at the faint end, one would expect the Stetson index to be correlated with source brightness, as seen in, e.g., Plavchan et al. (2008b) or CHS01. Figure 22 shows the distribution of all calculated Stetson indices against mean [3.6] (for the standard statistical sample, e.g., all objects with light curves, over all clusters, fast cadence only). Unlike the analogous figures found in, e.g., Plavchan et al. (2008b) or CHS01, here we have no substantial change in the bulk of the distribution of the Stetson index toward fainter [3.6] magnitudes, so we do not appear to have correlated noise 


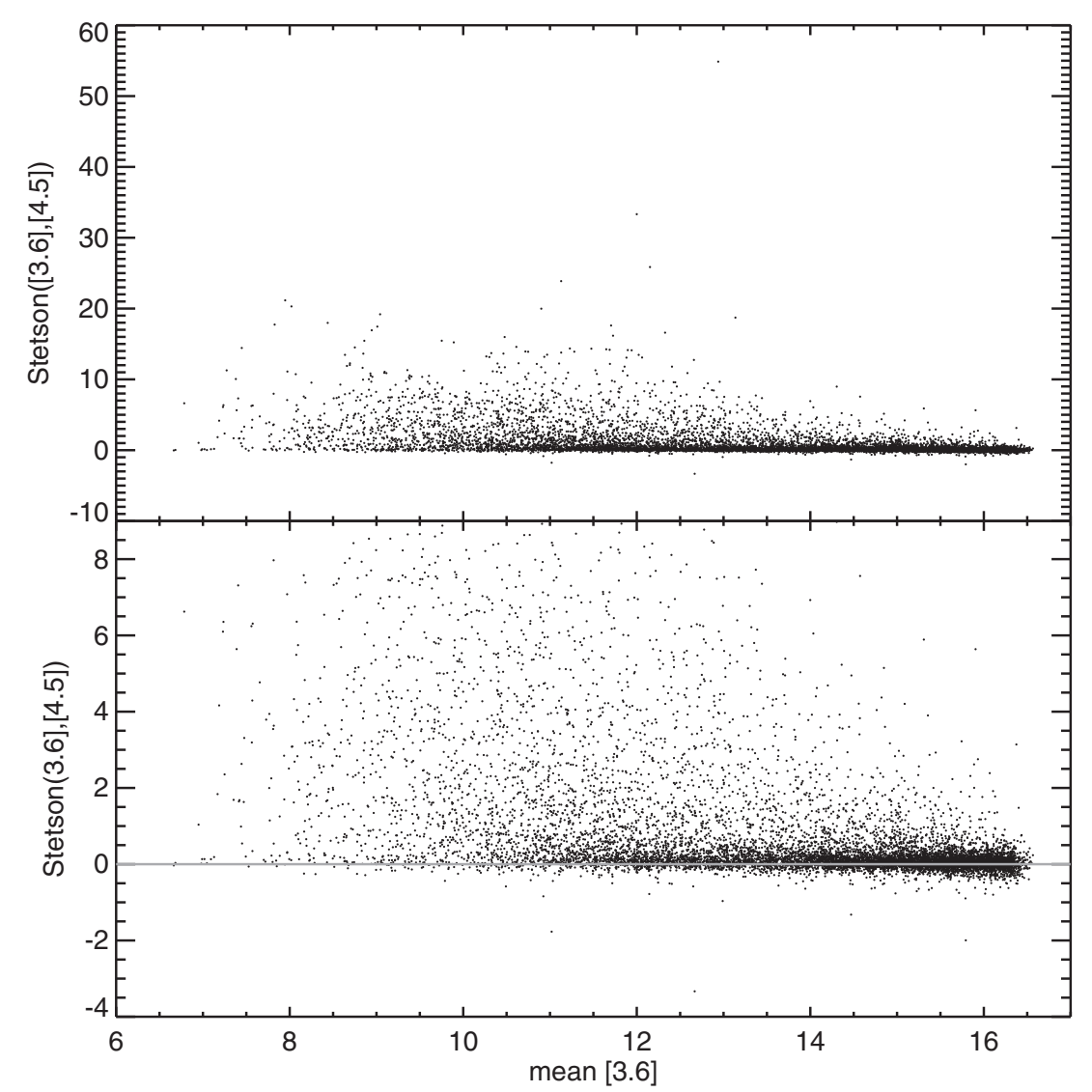

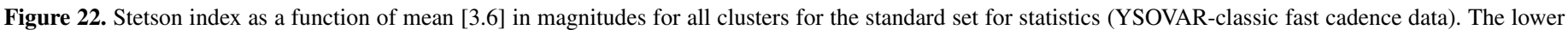

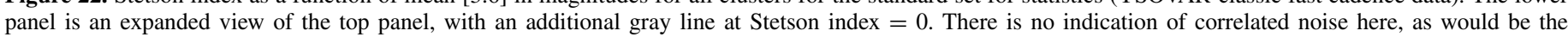

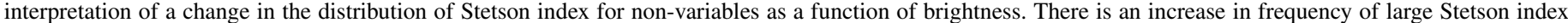

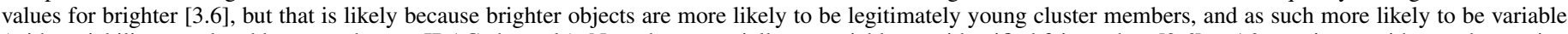

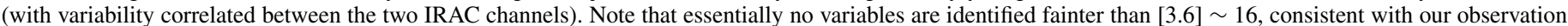
that those light curves are particularly noisy.

between the two channels. There is an increase in frequency of large Stetson index values for brighter [3.6], but that is likely because brighter objects are more likely to be legitimately young cluster members, and, as such, are more likely to be variable (with variability correlated between the two IRAC channels). Essentially no large values of the Stetson index are identified for objects fainter than [3.6] 16; the intrinsic error on each point is sufficiently large that these objects do not have a large Stetson index. (This is consistent with the discussion in Section 4.2 regarding the number of objects in our data set falling off rapidly fainter than $[3.6]-[4.5] \sim 16$.)

The specific location of the cutoff between variable and nonvariable can be unique to each data set, as it is affected by the sampling length and rate of the light curves. This is the primary reason behind our decision to calculate statistics over only the fast cadence window. We now discuss how we chose this cutoff value for the Stetson index. The left panel of Figure 23 shows a histogram of the Stetson indices for all objects in the standard set for statistics having at least five points in both I1 and I2. The bulk of the distribution about 0 are the non-variables, and that part of the distribution can be reasonably well-fit by a Gaussian. There are substantial deviations from Gaussianity toward higher values of the Stetson index, as expected for a population of identified variable stars. There is a change in the distribution of the Stetson index above and below 0.9; from where the distribution deviates from a Gaussian to a Stetson index of 0.9, the slope in the middle panel of Figure 23 is $\sim-1.0$, and from a Stetson index of 0.9 to $\sim 3$, the slope is $\sim-0.3$. Based on this, we take 0.9 as the cutoff for variability in our data set. The value of 0.9 corresponds to about $6 \sigma$ for the Gaussian fit to the distribution.

We note that in the analogous histograms for each individual cluster, each typically has a small gap in the Stetson index distribution at $\sim 0.9$. Orion, however, does not, and Orion contributes about half of the $\sim 11,000$ viable two-band light curves for which the Stetson index appears in Figure 23.

To check whether the Stetson index cutoff of 0.9 is sensible, we conducted a series of Monte Carlo tests. For random light curves (with a Gaussian distribution of points) using the same time sampling as the real data, the distribution of Stetson indices is well-described by a Gaussian with a width typically of $0.1-0.2$, comparable to the left-hand side of Figure 23. For Orion, where we have a reasonably welldefined set of members and non-members (from MC11; not just disked and non-disked, but confirmed membership lists), we can compare the distributions of Stetson indices for the members and non-members. In Orion, the distribution for non-members is generally fairly well-described by a Gaussian centered on 0 , but there is a small "shoulder" asymmetry toward the larger Stetson index (likely legitimate field variables or as of yet unidentified members). The distribution for members, in contrast, is not well-described by a Gaussian. It is asymmetric with a substantial excess of objects with high Stetson values. This is as expected, since members are more likely to have large amplitude, correlated variability. 

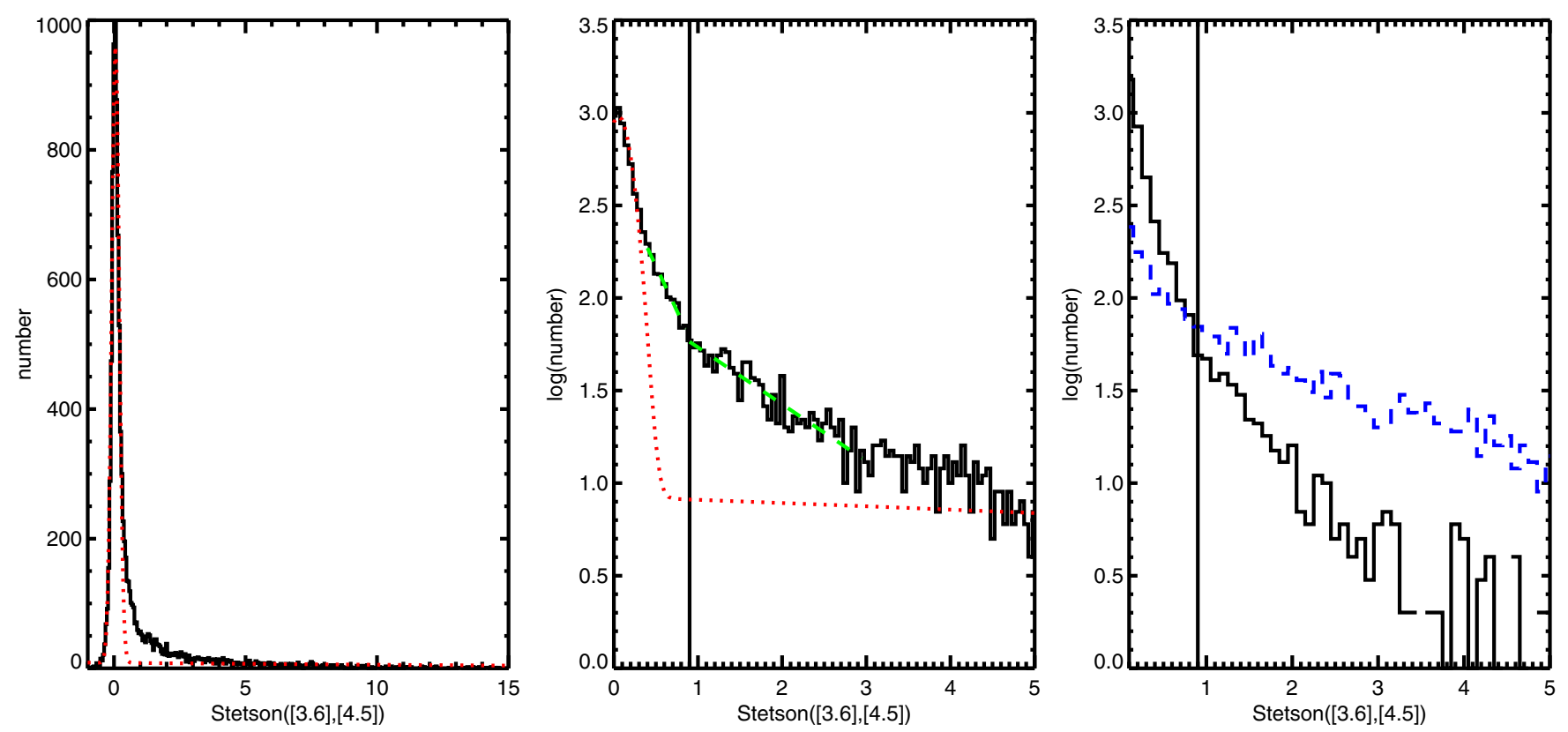

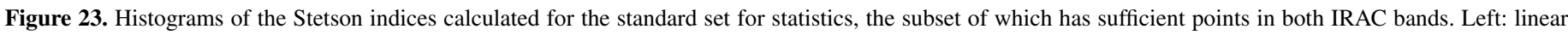

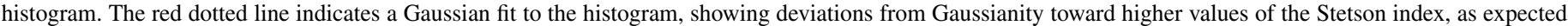

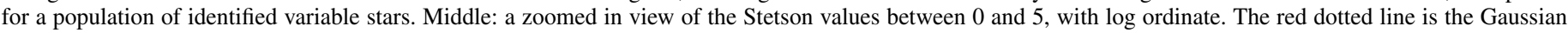

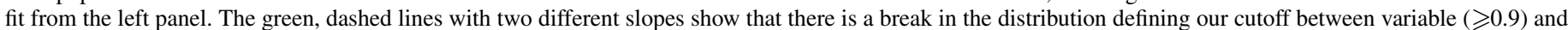

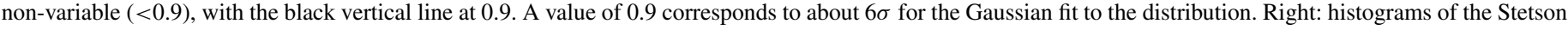

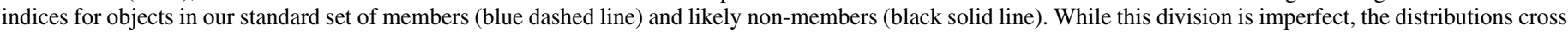

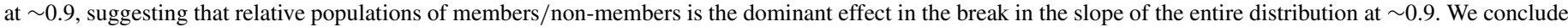
that the Stetson index cutoff of 0.9 is indeed a sensible boundary for demarcating variables from the general population.

(A color version of this figure is available in the online journal.)

Similarly, we can examine the distribution of the Stetson index for our standard set of members that are also in the standard set for statistics (and having sufficient points in both bands) and compare it to the Stetson index distribution for the remaining objects not selected as members (but still in the standard set for statistics, and having sufficient points in both bands). We obtain a similar result in the right panel of Figure 23; the Stetson distribution for members crosses that for non-members at about 0.9 , or perhaps a little below that level, suggesting that this division is the dominant cause of the break in the entire distribution at about that level. Even if our separation between members and non-members is imperfect (which it certainly is), these distributions are consistent with our selection of 0.9 as the cutoff. We conclude that a Stetson index cutoff of 0.9 is a sensible boundary for our data set.

While the objects with Stetson indices of $\lesssim 0.4$ have a very low chance of having legitimate correlated variability, and objects with Stetson indices $>0.9$ have a high likelihood of correlated variability, there is a continuum between these values. Objects with Stetson indices $\gtrsim 0.4$ and $\lesssim 0.9$ have lowconfidence for correlated variability. In MC11, we took a different Stetson index of 0.55 as the cutoff, based on the distribution of Stetson indices for that particular data set. As such, some of the identified low-confidence variables may have changed between this and the initial analysis. We also note that the cutoff in Stetson index for CSI 2264 is very different (Cody et al. 2014), but that program has a substantially different observing cadence than the YSOVAR-classic data discussed here. In general, the appropriate Stetson index cutoff must be determined for the individual data set, and there is no universal value.

\subsection{Chi-squared Test}

A second method to identify variables is a chi-squared test $\left(\chi^{2}\right)$, which, for a given band, is given by

$$
\chi^{2}=\frac{1}{N-1} \sum_{i=1}^{N} \frac{\left(\operatorname{mag}_{i}-\overline{\mathrm{mag}}\right)^{2}}{\sigma_{i}^{2}},
$$

where $\sigma_{i}$ is the estimated photometric uncertainty (corrected as per our discussion of the YSOVAR noise floor in Section 2.5).

This test is used to identify objects with uncorrelated variability, or variability in only one band (perhaps because data exist in only one band). This makes the $\chi^{2}$ test more susceptible to instrumental issues affecting only one band. However, to demonstrate that it generally does a good job of recovering variables with large Stetson indices, Figure 24 shows the distributions of $\chi_{I 1}^{2}$ and $\chi_{I 2}^{2}$ as a function of Stetson index. For those objects in the standard set for statistics where it is possible to calculate both $\chi^{2}$ and the Stetson index, the values are reasonably well correlated for the unambiguously variable objects. Using this plot, we find that a limit of $\chi_{I 1}^{2}$ or $\chi_{I 2}^{2} \sim 5$ is an appropriate conservative cutoff for potential variability in those cases where only one $\chi^{2}$ can be calculated (e.g., where monitoring in only one band is available).

For our largest data set (Orion), there are thousands of light curves that meet the requirement imposed by the Stetson index of having data in both IRAC channels. However, in the 11 smaller-field YSOVAR-classic data sets, imposing such a twoband restriction typically means that more than half the viable light curves would be discarded. Thus, the $\chi^{2}$ test is particularly useful in these cases where only one band is available. 


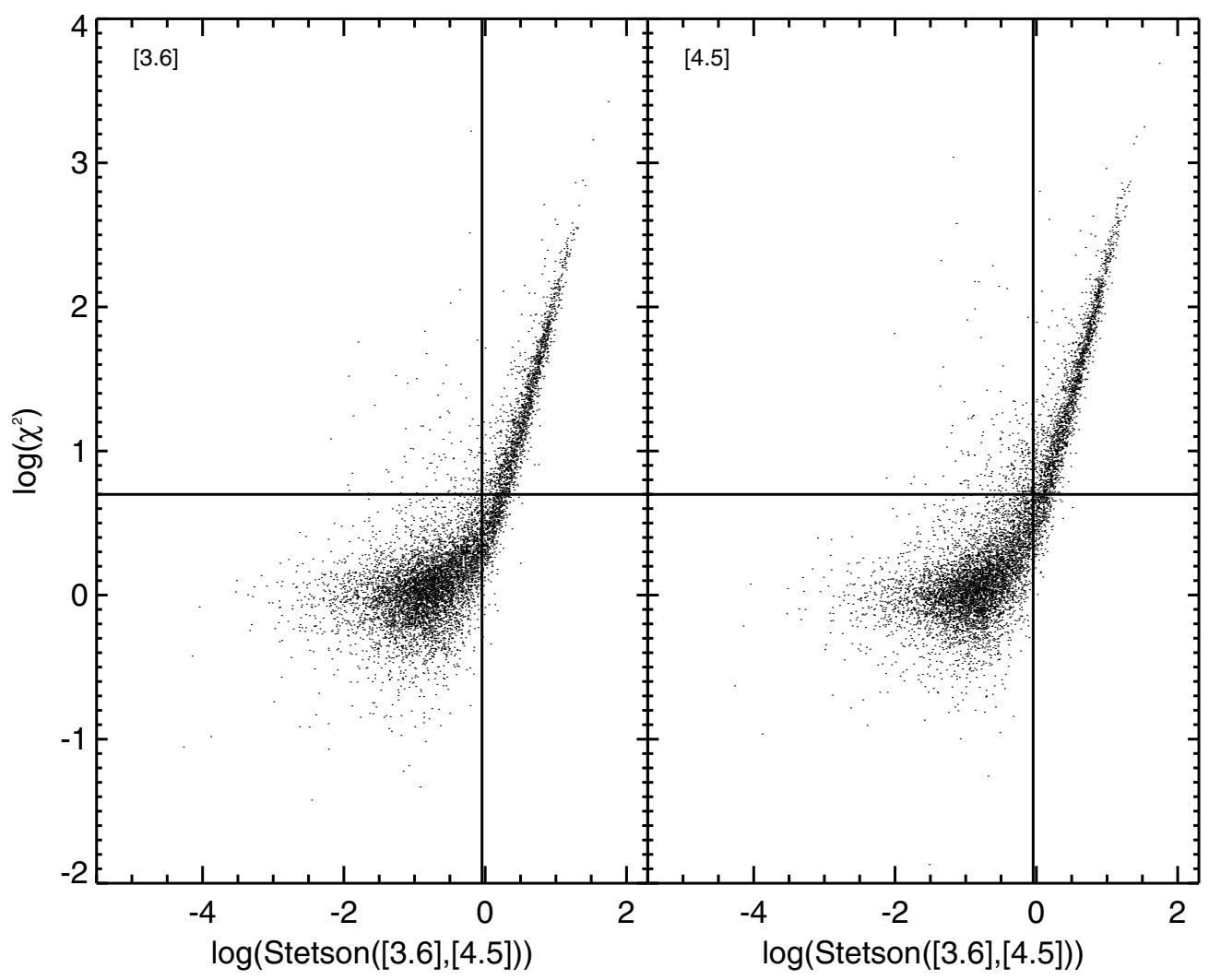

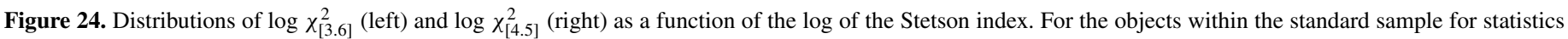

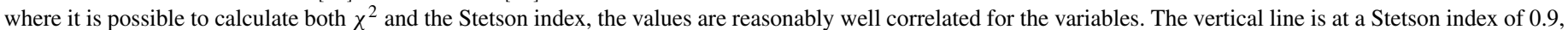

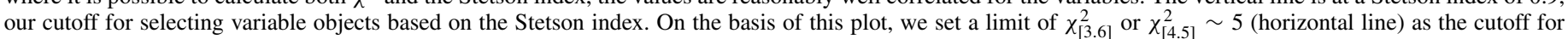
potential variability in those cases where only one $\chi^{2}$ can be calculated (e.g., where monitoring in only one band is available).

Figure 25 shows histograms of $\chi_{I 1}^{2}$ and $\chi_{I 2}^{2}$ for the standard sample for statistics, as well as for the subset of objects for which a Stetson index can be calculated (e.g., the sample used in the prior figure), and the smaller subsample of objects identified as variable using the Stetson index (Stetson index $>0.9$ ). We fit a Gaussian to the sharply peaked distributions, and found a $3 \sigma$ value of $\chi^{2} \lesssim 4.5$ in all cases. The bulk of the $\chi^{2}$ distribution for which the Stetson index is $>0.9$ also has $\chi^{2}>5$. To be conservative, we thus set a limit of $\chi_{I 1}^{2}$ or $\chi_{I 2}^{2}=5$ for identifying a candidate variable object. In the ideal case where there are multiple bands of monitoring data, one could assess each light curve with a large $\chi^{2}$ but small Stetson index for physical plausibility. To attempt to avoid identifying false variability due to instrumental effects, the light curves for each of these potentially variable objects will be examined by hand in the context of the individual cluster analysis to come in separate papers.

\subsection{Identifying Periodic Variables}

Finally, as in MC11, there are still legitimately variable sources within our standard statistical sample that fail both the Stetson index test (perhaps because only one band is available) and the $\chi^{2}$ test (perhaps because the amplitude of variability is small and our limits for identifying variability were conservative by design). There are many mathematical tools available for identifying periodic behavior in an unevenly sampled time series. The last test for variability we run here is a periodogram analysis using the NASA Exoplanet Archive
Periodogram Service ${ }^{34}$ (Akeson et al. 2013). This service provides period calculations using Lomb-Scargle (LS; Scargle 1982), Box-fitting Least Squares (BLS; Kovács et al. 2002), and Plavchan (Plavchan et al. 2008b) algorithms. These methods are varyingly more or less sensitive to periodic behavior shaped like sinusoids or flat-bottomed transits, and/or may be less sensitive to periodic behavior appearing in addition to other behavior, such as a period superimposed on a long-term trend. The expected periodic variability in our sample includes anything repeated, from a sinusoidal-like signal originating from hot or cool spots on a photosphere, to signals characteristic of close binaries, to repeated dips in the signal (like "dippers" or AA Tau; see, e.g., MC11), or even pulsations (e.g., Morales-Calderón et al. 2009).

Specifically, because of the variety of expected light curve shapes, and the weaknesses inherent in any of these methods for finding periodicity, and noting the approach used by (and results from) McQuillan et al. (2013a, 2013b), we also calculated the ACF for each light curve as a check on repeated patterns. We linearly interpolated the light curve onto evenly spaced times, and then calculated the ACF using the following expression where $L$ is a lag in days, and $x$ is the light curve (with elements $x_{k}$ ):

$$
\operatorname{ACF}_{x}(L)=\operatorname{ACF}_{x}(-L)=\frac{\sum_{k=0}^{N-L-1}\left(x_{k}-\bar{x}\right)\left(x_{k+L}-\bar{x}\right)}{\sum_{k=0}^{N-1}\left(x_{k}-\bar{x}\right)^{2}} .
$$

\footnotetext{
${ }^{34}$ http://exoplanetarchive.ipac.caltech.edu/cgi-bin/Periodogram/nphsimpleupload
} 


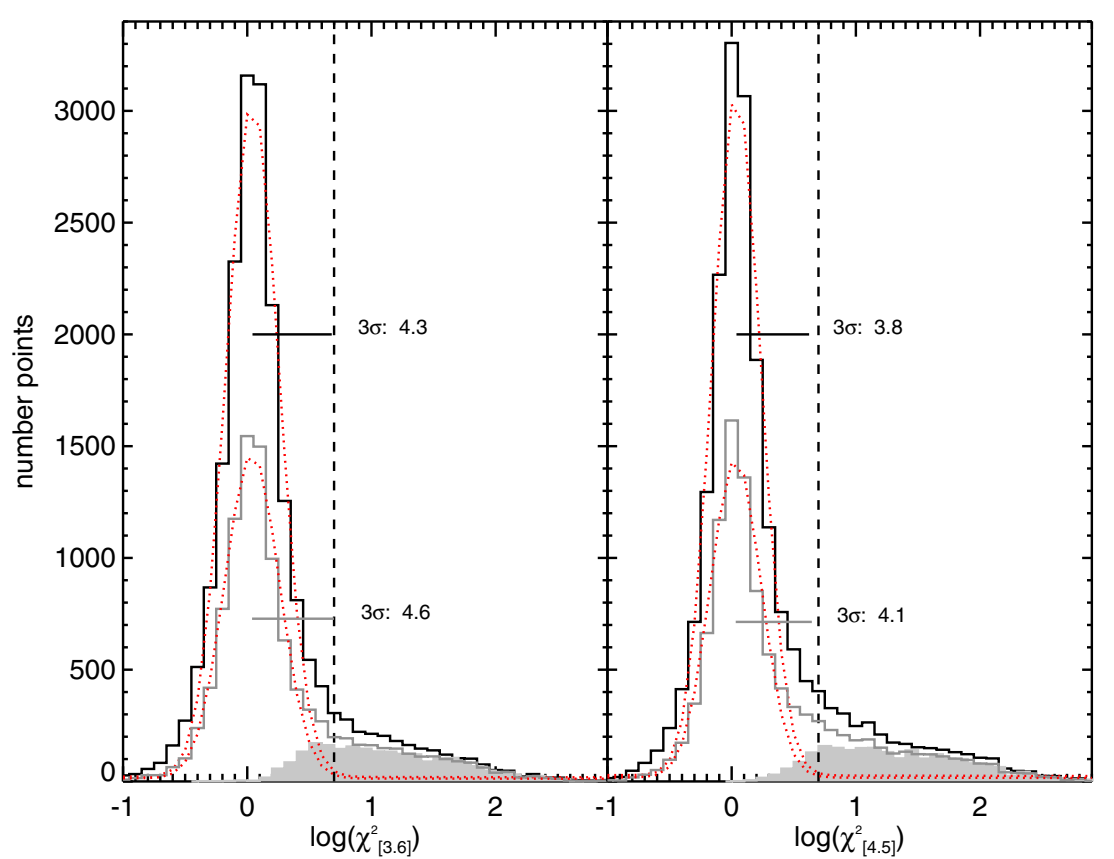

Figure 25. Distributions of $\log \chi_{[3.6]}^{2}$ (left) and $\log \chi_{[4.5]}^{2}$ (right) for all objects with $\chi^{2}$ values (black solid line histogram), for those objects with $\chi^{2}$ and Stetson indices (gray solid line histogram), and for those objects with $\chi^{2}$ and a Stetson index $>0.9$ (light gray filled histogram). (All objects shown here are from the standard set for statistics.) The red dotted line is a Gaussian fit to the corresponding histogram. The $3 \sigma$ values corresponding to that Gaussian, converted to linear $\chi^{2}$, are indicated. We identify a limit of $\chi_{[3.6]}^{2}$ or $\chi_{[4.5]}^{2} \sim 5$ as a conservative cutoff for potential variability in those cases where only one $\chi^{2}$ can be calculated (e.g., where monitoring in only one band is available). That limit is plotted as the vertical dashed line. We take objects with $\chi_{[3.6]}^{2}$ or $\chi_{[4.5]}^{2}>5$ as legitimately variable.

(A color version of this figure is available in the online journal.)

We experimented with several different timescales as obtained from the ACF, and settled on the location of the first peak, providing that the peak was above an ACF value of 0.2 . For those objects with significant periods, this coherence time should be well-matched to the period.

We looked for periods in light curves where we had at least 20 points (more restrictive than our standard statistical sample); we ran all four methods (LS, BLS, Plavchan, and ACF) on not just the $3.6 \mu \mathrm{m}$ and $4.5 \mu \mathrm{m}$ light curves, but also, where possible, the [3.6]-[4.5] light curves. In some cases, a long-term trend (astrophysical, not instrumental) is present in the individual I1 or I2 light curve, masking a periodic signal, but the color exhibits the periodic signal. We looked for periods between only 0.05 and 15 days, given the overall sampling of our data, and we require at least two complete periods over the typically $\sim 40$ day window of our observations. We investigated phased light curves for those periods calculated using all of these methods. Based on these many thousands of results, we concluded that LS is the best, for our data set, for finding reliable, plausible periods. BLS and the Plavchan algorithm, while they look for a wider variety of shapes of signals, struggle with light curves that typically have less than 100 points, as ours do; the ACF approach finds only the strongest signals. Typically, if the LS algorithm found a reliable period, those other three approaches found comparable periods.

Thus, we filtered first on the LS results. We excluded candidate periodic objects if the calculated false alarm probability (FAP; see, e.g., Scargle 1982) was $>0.03$, or if the recovered period was $>14.5$ days and the FAP for that period was $>0.01$, or if the period was $<0.1$ day (slightly larger than the lower limit over which we searched), or if the calculated period was exactly 15.0 days (by inspection of the light curves, input and phased, a returned period exactly equal to the upper limit of our search window was usually indicative not of a true periodicity, but instead of a long-term trend in the data). For each of the remaining objects, we investigated the phased light curve. Perhaps unsurprisingly, given our overall FAP cutoff of 0.03 , about $3 \%$ of the surviving candidate periodic light curves did not produce physically plausible phased signals. Those objects were omitted from the final set of periods, and will be identified as such in the corresponding cluster papers. The planned individual cluster papers may include a few additional periodic objects not automatically identified due to the presence of outlying photometry points which mask periodicity unless removed by hand.

We proceed to include all of the periodic objects identified via the LS algorithm (excluding the candidate periodic objects as described) in the set of variable objects, even if they fail the other (Stetson, $\chi^{2}$ ) variability tests. Individual objects will be discussed in the papers dedicated to each individual cluster, but anywhere from 1 to 15 objects, typically $\leqslant 5$, were added to the list of likely variables for each of the smaller-field clusters. For any given object, we wish to assign a single period to that object. We take any period derived from the [3.6] data first ([3.6] is less noisy than [4.5]), then, only if there is no [3.6] period of sufficient power, we take the period derived from [4.5], and finally, if no other period of sufficient quality is available, then we take that derived from [3.6]-[4.5].

A preliminary list of periodic objects in Orion appeared in MC11. The approach we are now using to search for periods is more stringent than that in MC11. Our current approach recovers the bulk of the objects that MC11 reported as periodic, but does not, for example, recover the objects reported as having $P>15$ days. A complete list of the $\sim 800$ periodic variables in Orion as derived from this YSOVAR data reduction will appear in a later paper. 


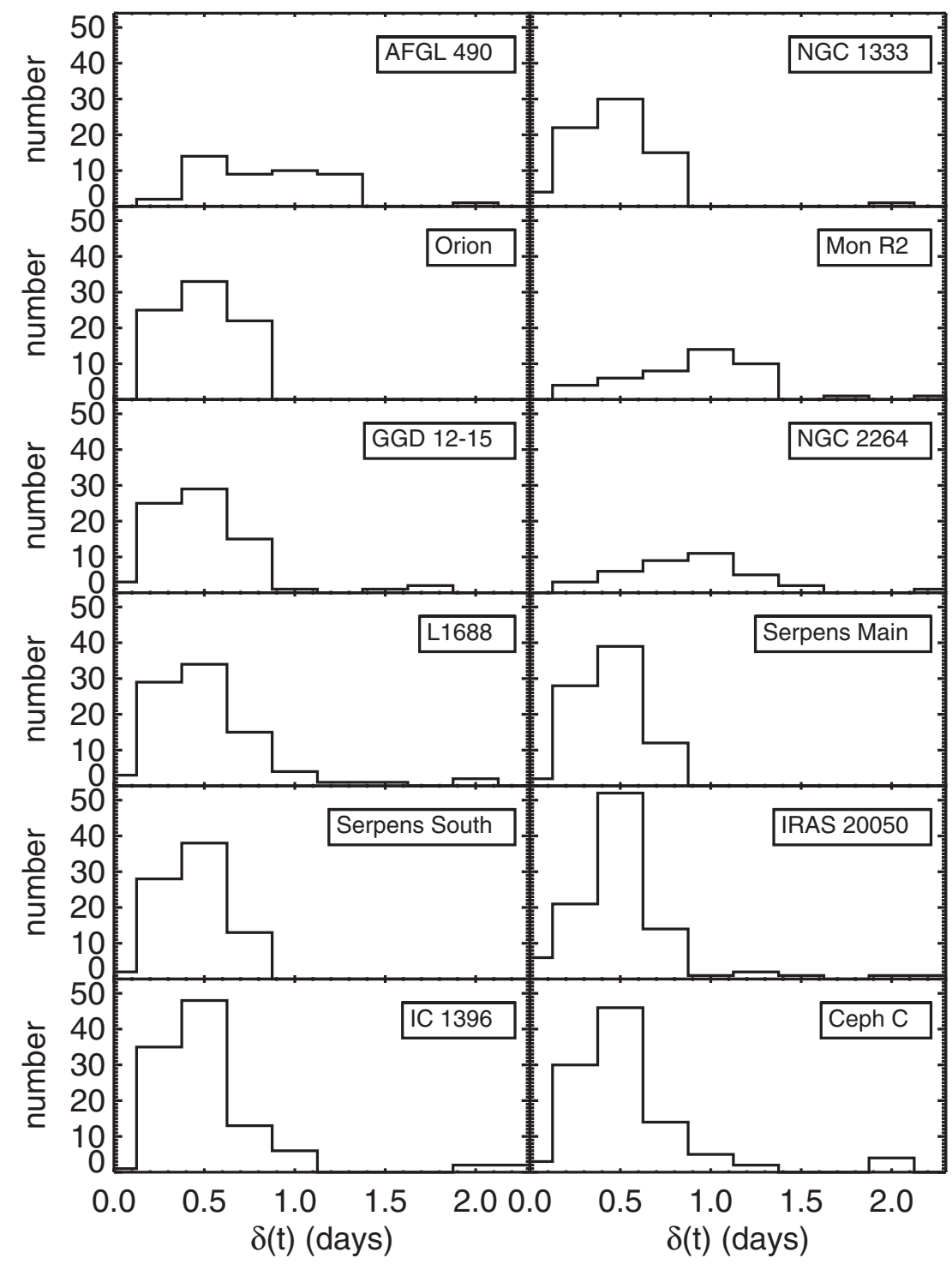

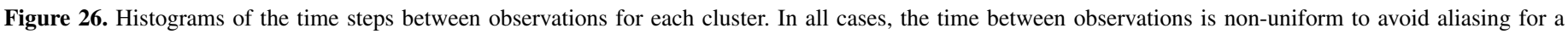
specific period. AFGL 490, Mon R2, and NGC 2264 have a lower overall sampling rates than all other clusters.

\subsection{Detection Limits for Variability}

Above, we described how variable sources are identified using the Stetson test, the $\chi^{2}$ test, and a search for periodic variability. The cut-off values for those tests were chosen to yield a conservative list of variable sources and to reject those sources where the variability stems mostly from instrumental artifacts. We now present Monte-Carlo simulations to quantify how much variability is required to meet those criteria.

Several different physical effects may contribute to the observed variability and this can lead to very complex patterns in the light curve. In the absence of a theoretical model to explain the different contributions, we concentrate on simple analytical prescriptions for light curves so as to gain a sense of the sensitivity of our statistical tools. First, we consider a source that has two states, a bright and a faint state. The light curve switches randomly between those two states. We simulate light curves for a different fraction of time spent in the upper state $(0 \%, 10 \%$, $20 \%, 30 \%, 40 \%$, and $50 \%$ ) and we expect that variability is more easily found for sources with a larger amplitude between the two states and an equal chance to find the source in each state. Second, we simulate sinusoidal light curves with different periods-0.1-2 days (in steps of 0.1 day) and 2-20 days (in steps of 2 days). We add Gaussian noise to each light curve and vary the ratio of the signal amplitude and the noise (from 0 to 10 in steps of 0.1 , where a relative amplitude of 0 means a constant light curve with noise only). The sensitivity of the Stetson test, the $\chi^{2}$ test, and the Lomb-Scargle periodogram is independent of the magnitude of a source (that is not noise-dominated); only the relative amplitude of the signal and the noise plays a role. Thus, it is equally possible to detect strong variability in a weak source (with large photometric uncertainties) as weak variability in a bright source (with small photometric uncertainties).

For each grid point in relative amplitude and fraction of time in the upper level or period, for each band, we simulate 10,000 light curves for each grid point for each band. The light curves are sampled at the time intervals of the actual YSOVAR observations. Figure 26 shows the histogram of the time steps between observations for each cluster (the typical min and max $\Delta t$ were given above in Table 3 ). For all clusters, the sampling is non-uniform to avoid aliasing for a specific period. However, the histograms fall in two groups, with AFGL 490, Mon R2, and 


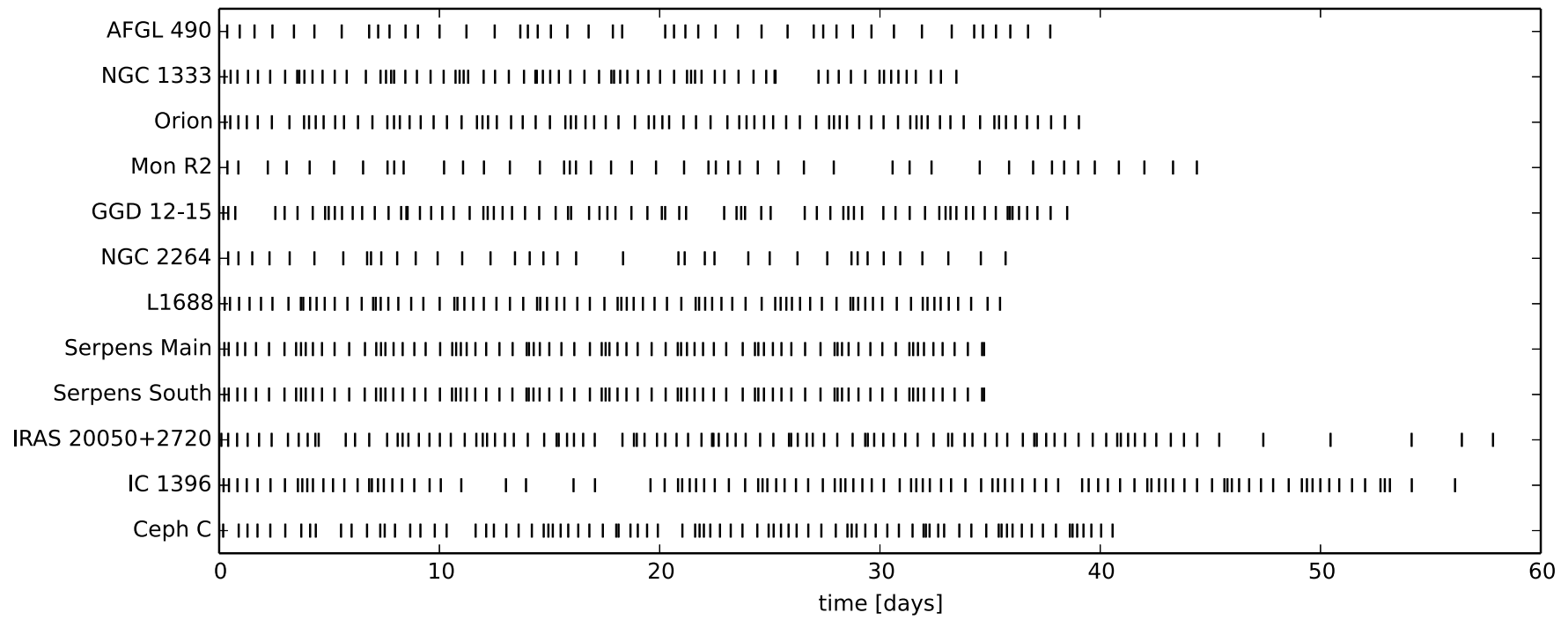

Figure 27. Representation of the relative time steps for the fast cadence observations, in a similar format to Figure 14, with a "l" denoting a time step (from the red sections of Figure 14). AFGL 490, Mon R2, and NGC 2264 can also be seen here as having a lower overall sampling rates.
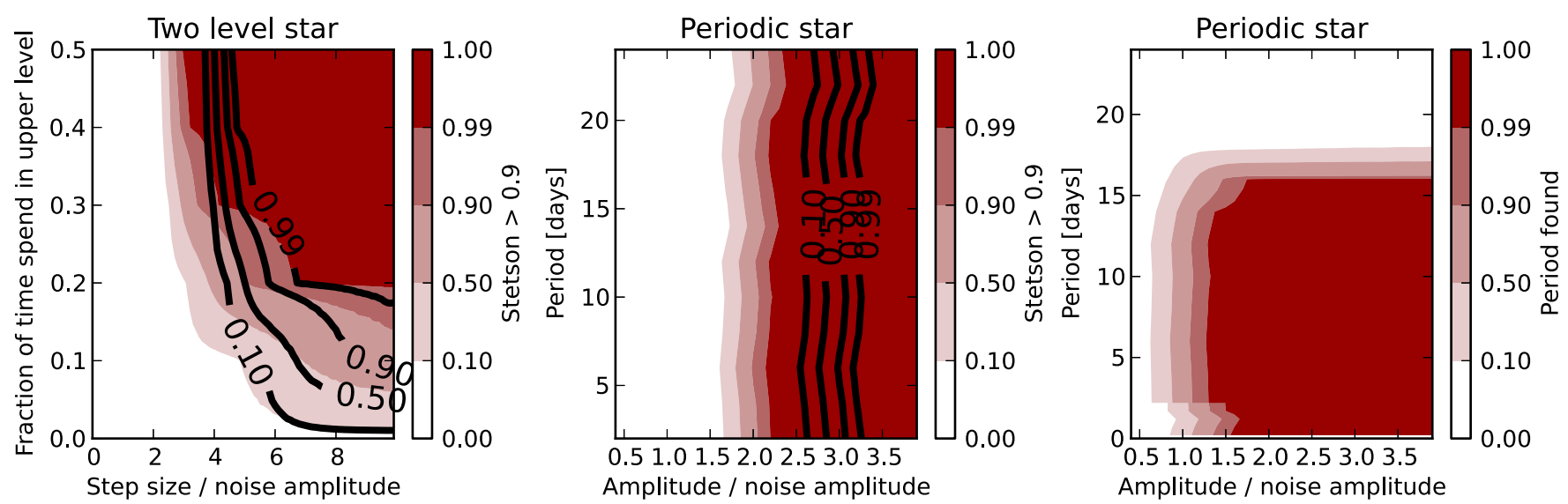

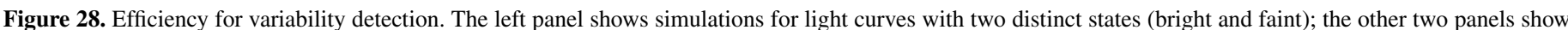

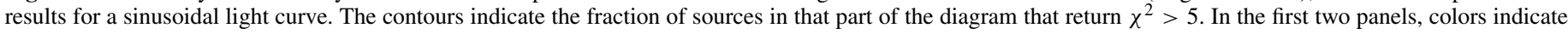

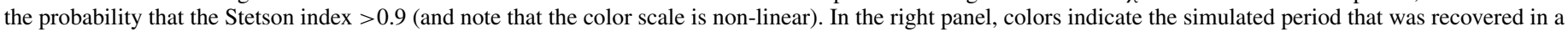
Lomb-Scargle periodogram. The time sampling is from the observations of L1688.

(A color version of this figure is available in the online journal.)

NGC 2264 having a lower overall sampling rate than the other clusters. Figure 27 is another representation of the sampling rate. In the style of Figure 14, it visually represents the sampling rates for the fast cadence monitoring. Here, AFGL 490, Mon R2, and NGC 2264 can also be seen to have a lower overall sampling rate than the other clusters. We ran all Monte Carlo simulations using the actual time sampling from L1688, one of the clusters with a high sampling rate and Mon R2, a representative cluster with a lower sampling rate.

Figure 28 shows results from the Monte Carlo simulations using the sampling of the L1688 cluster. The left panel presents the detection efficiency for light curves from an object with two distinct luminosities. If the star is found in each state half the time, the Stetson test will identify it as variable in almost all cases $(99 \%)$ if the step size is at least three times larger than the noise level. Since the $\chi^{2}$ test uses data from one band only, the step size must be larger (five times the noise level) to reach the same detection efficiency. If the star spends less than $20 \%$ of the time in either state, there is a reasonable chance that the variability will not be found, even for larger step sizes, since the sampling might find only few data points in this state.
For a periodic light curve (middle panel), variability is again found more easily in the Stetson test than in the $\chi^{2}$ test, and the period of the variability does not influence the detection efficiency, since those two tests do not consider the time ordering of the observed data. Using the LS approach, we are sensitive to periods $(P)$ between about 1 and 15 days. Even periods where the amplitude $(a)$ of the light curve $a \sin (2 \pi / P t)$ is only twice as large as the noise level are easily detected, almost independent of the period in the range 1-15 days. Such weak signals would not necessarily show up as variable in the Stetson or $\chi^{2}$ test (see the middle panel). In general, the region where the tests detect variability in some light curves but not in others is fairly narrow.

Figure 29 shows the same plots as Figure 28 but for the time sampling of Mon R2, one of the clusters with a lower cadence in the observations. The general shape of the regions in the parameter space where variability can be detected is the same, but, due to the lower number of observations and the larger time span between observations, a larger amplitude is required, and we are less sensitive to periods below about two days.

To explore more complicated light curves, we combined several effects, e.g., a sinusoidal periodicity overlaid on a 

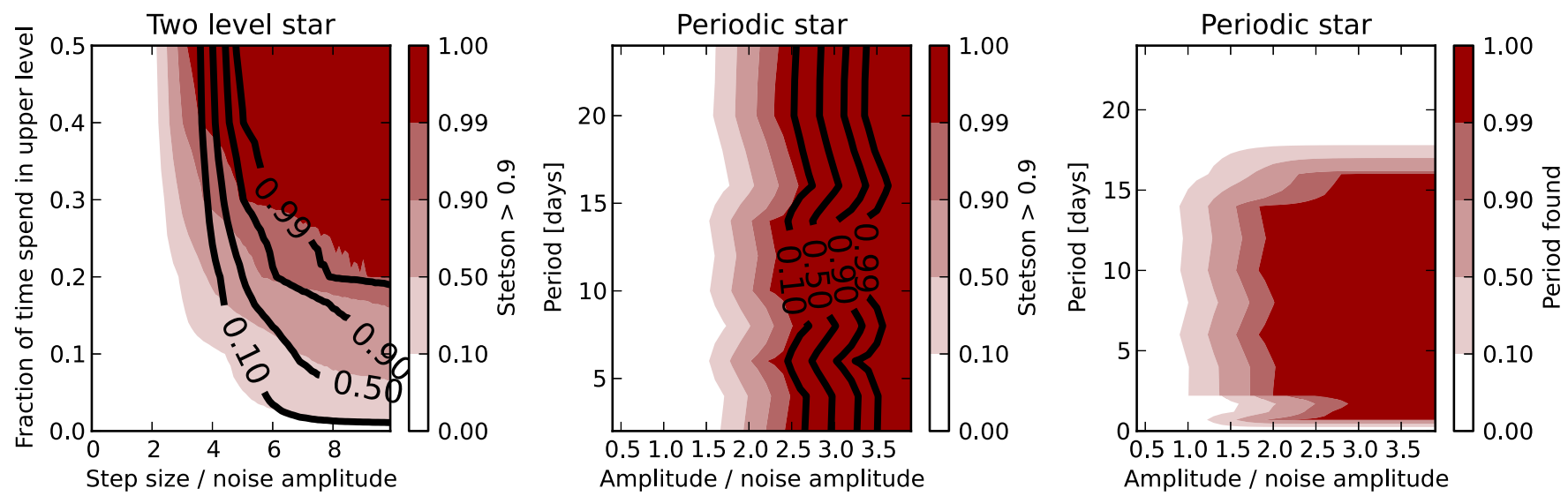

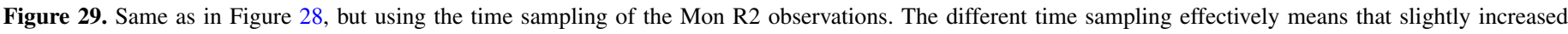
signal-to-noise ratios are required.

(A color version of this figure is available in the online journal.)

long-term trend. In these cases, the strongest effect generally determines how the variability will be seen. If the magnitude of the trend is large and the amplitude of the sine wave is small, then the light curve will be marked as variable, but the periodicity might not be detected.

Given the possible complexity of real light curves, it is not possible to cover the entire parameter space with Monte Carlo simulations. However, the scenarios presented here show that, in general, we can detect periodicity with an amplitude just twice the level of the noise, variability at 3-5 times the level of the noise with the Stetson test and about 6-10 times the level of the noise for single-band light curves with the $\chi^{2}$ test. Similar results for the relative sensitivity of the Stetson and the $\chi^{2}$ test were also found by Flaherty et al. (2013), although they used different cut off levels than this work.

As seen above in Figure 16 and in MC11, there is a reasonably strong correlation between the mean magnitude of a source and its mean error. This overall relation affects our ability to find variability or periodicity. For sources brighter than 13th mag, the total uncertainty is dominated by the error floor introduced in Section 2.5. For those brighter sources, we can detect periodicity if the amplitude is larger than about 0.02 mag and variability if it is larger than about $0.03-0.1 \mathrm{mag}$ (with the exact number depending on the signal shape).

\subsection{Identifying Variables: Cryo-to-post-cryo (Six to Seven Years)}

In addition to the variability probed on the YSOVAR monitoring timescale of $\sim 40$ days, we also are interested in the evidence for longer-timescale variations between observations of these same clusters in the Spitzer cryogenic epoch and the post-cryo (YSOVAR) epochs. To identify the variables in this case, for every object with a light curve, we can compare the average measurement from the earliest cryo era (Table 4), and the mean for that object over the YSOVAR standard statistical sample (Section 3.2).

The process we used to identify the long-term variables in each cluster is shown for AFGL 490 in Figure 30. We plot the difference between the cryo and post-cryo measurements for each object as a function of the cryo value and determine, for each cluster, the brightness at which photometric noise clearly dominates. This faintness limit is close to 16th mag, consistent with what we noted in Figures 19-22. We select this limit separately for each cluster and consider only objects brighter than this limit. We fit a Gaussian to the histogram of the difference between the cryo and post-cryo measurements, allowing the zero point as well as the height and width of the Gaussian to be free parameters. We classify as long-term variables all objects with cryo to post-cryo offset further than $3 \sigma$ from the peak of the (fitted) distribution. All objects from the standard statistical sample, not just the members (or only the variables), go into this process of defining the width of the distribution. The fraction of objects in each field that are classified as variable, and the subset of variables that are also cluster members, are both identified after the long-term variables are identified. A summary of the important parameters in these analysis steps to search for variables over this long-term baseline is in Table 7; the values we used for $\Delta t$, the time lapse between the cryo and post-cryo observations, are included in Table 4.

\section{DISCUSSION}

In this section, we present an analysis of the distribution of rotation rates as a function of IR excess, evidence (or lack thereof) for transient IR excesses, evidence for skews over time toward more brightening or fading sources, and how the long-term variability fraction varies as a function of cluster parameterization (from Section 4.1) or length of time baseline sampled.

\subsection{Periodic Variables}

Our YSOVAR map in Orion is far larger than the other cluster maps, which focus on the most embedded (possibly youngest) objects in these clusters. For these embedded objects, it is not generally possible to obtain a rotation period from ground-based optical or NIR data due to extinction. Moreover, for stars with more significant disks, it is less likely that the IR light curve will be strictly periodic. Many distinct processes can contribute to a YSO's mid-IR variability, and it often results in a stochastic light curve (Cody et al. 2014).

For the objects for which we can derive a period, we would like to compare our values to those from the literature as a check on our methodology. Since our clusters are, for the most part, very embedded, there are not very many known periods in the literature. As discussed in Section 2.1.2, there are many periods for Orion and NGC 2264, typically obtained in the optical, but with some values from the NIR. Parks et al. (2014) reports on NIR periods from objects in our region of L1688; there are other 

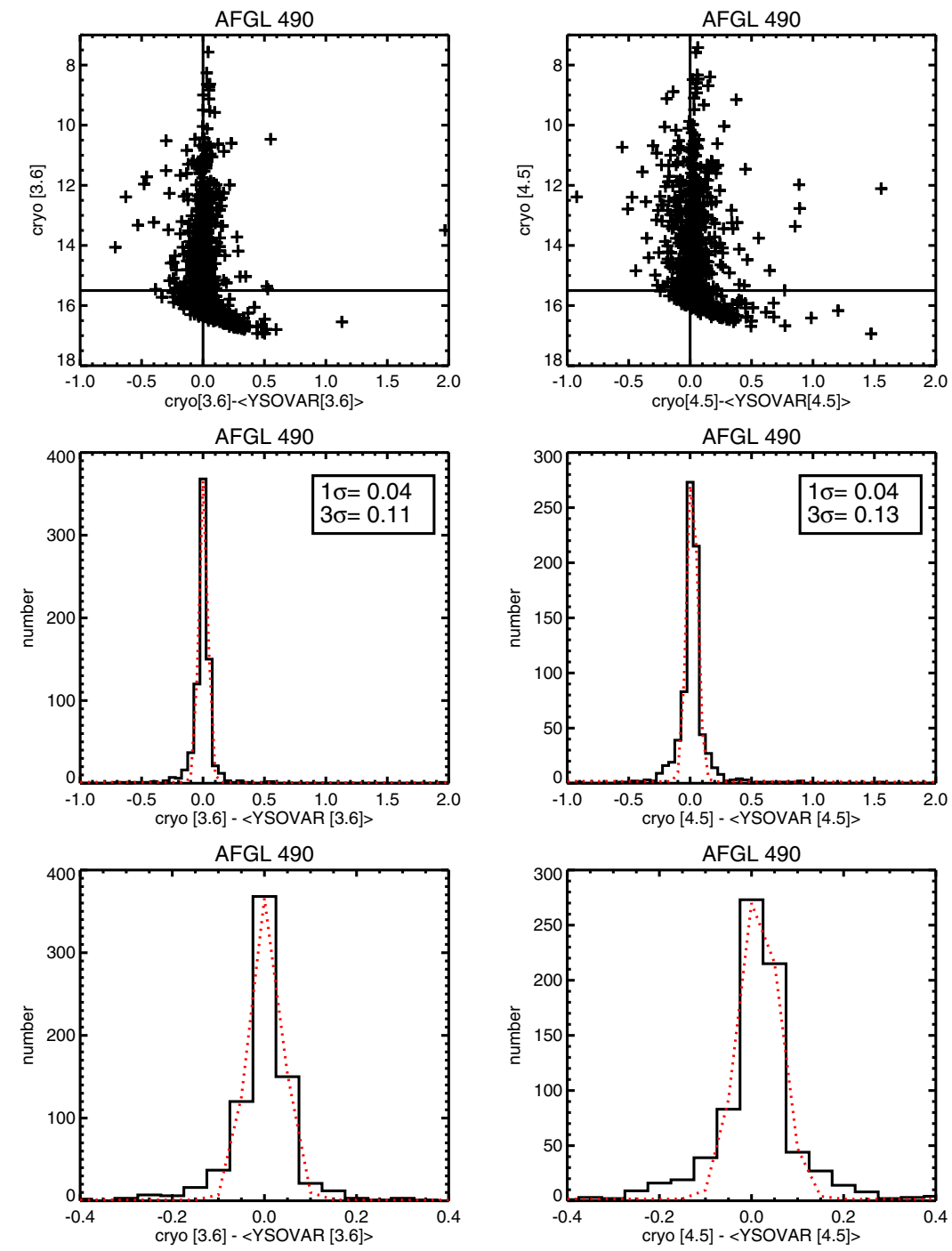

Figure 30. Illustration of the process used to identify long-term variables in the clusters. Top: plot of the difference between the cryogenic-era measurement and the mean measurement from the standard set for statistics against the cryogenic-era measurement (left: [3.6], right: [4.5]). Only objects brighter than 15.5 were used (in this cluster) for the next step. Middle: histograms of the difference in magnitudes for objects brighter than 15.5. The solid line is the data; the dotted line is a Gaussian fit to the histogram. Based on this fit, we retained objects for which the difference in magnitudes is greater than $3 \sigma$ away from the peak of the distribution as likely variables. Bottom: zoomed in view of the central portions of the prior two histograms. A summary of this analysis searching for variables over this long-term baseline is in Table 7.

(A color version of this figure is available in the online journal.)

Table 7

Statistics on Variable Objects on the Longest Timescales

\begin{tabular}{|c|c|c|c|c|c|c|c|c|}
\hline Cluster $^{a}$ & $\begin{array}{l}\text { I1 Faint Cutoff } \\
\text { (mag) }\end{array}$ & $\begin{array}{l}\text { I1 Center } \pm \sigma^{\mathrm{b}} \\
(\mathrm{mag})\end{array}$ & $\begin{array}{c}\text { I1 Vars/LCs }{ }^{\mathrm{c}} \\
(\mathrm{mag})\end{array}$ & I1 Memb.vars/LCs ${ }^{\mathrm{d}}$ & $\begin{array}{l}\text { I2 Faint Cutoff } \\
(\mathrm{mag})\end{array}$ & $\begin{array}{c}\text { I2 Center } \pm \sigma^{\mathrm{b}} \\
(\mathrm{mag})\end{array}$ & $\begin{array}{c}\text { I2 } \operatorname{Vars} / \mathrm{LCs}^{\mathrm{c}} \\
(\mathrm{mag})\end{array}$ & I2 Memb.vars/LCs \\
\hline AFGL 490 & 15.8 & $0.001 \pm 0.04$ & $86 / 907=0.09$ & $43 / 120=0.36$ & 15.8 & $0.017 \pm 0.05$ & $112 / 904=0.12$ & $58 / 155=0.37$ \\
\hline NGC 1333 & 16.0 & $0.033 \pm 0.05$ & $43 / 289=0.15$ & $24 / 115=0.21$ & 16.0 & $0.035 \pm 0.05$ & $82 / 393=0.21$ & $31 / 112=0.28$ \\
\hline Orion & 16.0 & $0.008 \pm 0.04$ & $738 / 5313=0.14$ & $439 / 2326=0.19$ & 16.0 & $0.013 \pm 0.04$ & $920 / 5921=0.16$ & $476 / 2426=0.20$ \\
\hline Mon R2 & 16.0 & $0.020 \pm 0.06$ & $57 / 431=0.13$ & $32 / 170=0.19$ & 16.0 & $0.031 \pm 0.11$ & $24 / 211=0.11$ & $10 / 90=0.11$ \\
\hline GGD 12-15 & 16.0 & $0.014 \pm 0.05$ & $56 / 336=0.17$ & $35 / 127=0.28$ & 16.0 & $0.046 \pm 0.06$ & $44 / 400=0.11$ & $28 / 138=0.20$ \\
\hline NGC 2264 & 15.8 & $0.011 \pm 0.06$ & $47 / 365=0.13$ & $29 / 142=0.20$ & 15.8 & $0.017 \pm 0.09$ & $58 / 492=0.12$ & $27 / 199=0.14$ \\
\hline L1688 & 15.5 & $0.017 \pm 0.05$ & $34 / 264=0.13$ & $18 / 48=0.38$ & 15.5 & $0.039 \pm 0.06$ & $47 / 312=0.15$ & $13 / 43=0.30$ \\
\hline Serpens Main & 15.5 & $0.008 \pm 0.04$ & $130 / 1112=0.12$ & $25 / 61=0.41$ & 15.5 & $0.038 \pm 0.05$ & $135 / 1161=0.12$ & $24 / 83=0.29$ \\
\hline Serpens South & 15.5 & $0.013 \pm 0.04$ & $59 / 587=0.10$ & $28 / 68=0.41$ & 15.5 & $0.031 \pm 0.05$ & $80 / 706=0.11$ & $24 / 78=0.31$ \\
\hline IRAS $20050+2720$ & 15.8 & $0.004 \pm 0.05$ & $123 / 1372=0.09$ & $31 / 114=0.27$ & 15.8 & $0.021 \pm 0.06$ & $112 / 1415=0.08$ & $32 / 106=0.30$ \\
\hline IC 1396A & 15.5 & $-0.007 \pm 0.03$ & $117 / 1122=0.10$ & $21 / 73=0.29$ & 15.5 & $0.003 \pm 0.04$ & $185 / 2116=0.09$ & $44 / 175=0.25$ \\
\hline Ceph C & 15.8 & $0.004 \pm 0.04$ & $51 / 531=0.10$ & $27 / 71=0.38$ & 15.8 & $0.030 \pm 0.05$ & $61 / 553=0.11$ & $33 / 82=0.40$ \\
\hline
\end{tabular}

Notes.

${ }^{\text {a }}$ The values we used for $\Delta t$, the time laps between the cryo and post-cryo observations, are included in Table 4.

${ }^{\mathrm{b}}$ Mean I1 or I2 cryo-to-postcryo offset, e.g., center (peak location) and $\sigma$ of Gaussian fit to distribution of differences in magnitudes brighter than the faint cutoff.

${ }^{c}$ Number of identified long-term variables over all of the objects with light curves/number of light curves (for all objects) $=$ long term variability fraction for all objects.

${ }^{\mathrm{d}}$ Number of identified long-term variables over only the standard set of members/number of light curves (for members) $=$ long term variability fraction for members. 


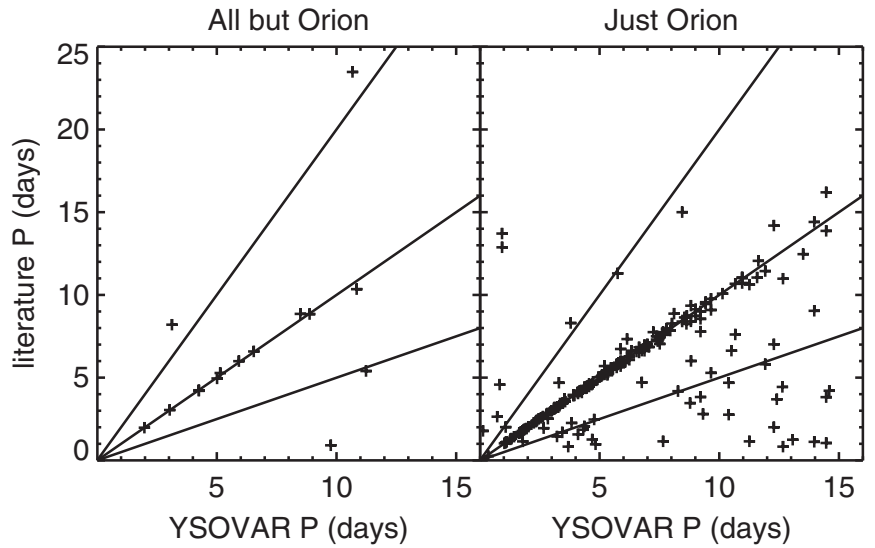

Figure 31. YSOVAR-determined period in days against the literature period in days for (left) NGC 2264, L1688, and IC 1396A, and (right) Orion alone. Solid lines indicate a 1:1 match, a 2:1 match, and a 1:2 match.

literature values for rotation periods of objects elsewhere in L1688, beyond our monitored region. We can roughly compare to the MIR timescales reported in Morales-Calderón et al. (2009) for IC 1396A.

Of the objects with periods in the literature, there are $\sim 200$ that also have periods derived here in Orion (excluding those from $\mathrm{MC} 11$, since those were derived from the same observations we use here), and an additional $\sim 15$ from NGC 2264, L1688, and IC 1396A. About $75 \%$ of those have period measurements that match to better than $10 \%$, so we have confidence that our period-finding approach is at least wellmatched to those in the literature. Figure 31 plots the YSOVARdetermined period against the literature period for those objects where it is possible. The clusters that are not Orion (NGC 2264, L1688, and IC 1396A) are plotted separately simply because Orion dominates the statistics, and it is useful to see if there are good matches outside Orion as well as within Orion. Three of the four objects from the three smaller-field clusters that are not well-matched to the YSOVAR-determined period are close to likely harmonics, and the periods are of comparatively low quality. The one that is most discrepant is from NGC 2264, SSTYSV 064101.40+093408.1, and is being compared to a period from Lamm et al. (2004). Our phased light curve looks correct (for our wavelength and epoch of observation). Of the $\sim 50$ Orion periods that do not agree to $10 \%$ (out of $\sim 200$ Orion period comparisons total), it is predominantly the case (by a ratio of 3 to 1 ) that the YSOVAR period is longer than the literature period. About $60 \%$ of these have [3.6]-[8] $>0.8$. All but five of those Orion periods were optically determined. Because we are working in longer wavelengths, it is possible that, particularly in those cases, we may be sampling a different location in the star-disk system, e.g., further away from the photosphere, where Keplerian rotation periods are longer.

A relation has already been found between IR excess and rotation rate for young stars, suggesting that IR excess and rotation rate are related; out of our 12 clusters, this relation has been found in Orion (Rebull et al. 2006) and NGC 2264 (Cieza \& Baliber 2007). In the 11 smaller-field clusters (i.e., all but Orion), there are $\sim 350$ stars with periods measured from YSOVAR light curves, but only $\sim 250$ of those also have cryogenic Spitzer measurements at 3.6 and $8 \mu \mathrm{m}$ from which we can get a clear indication of the IR excess in these systems. There are $\sim 800$ stars in Orion with measured YSOVAR periods, but only $\sim 430$ have [3.6] and [8] measurements.

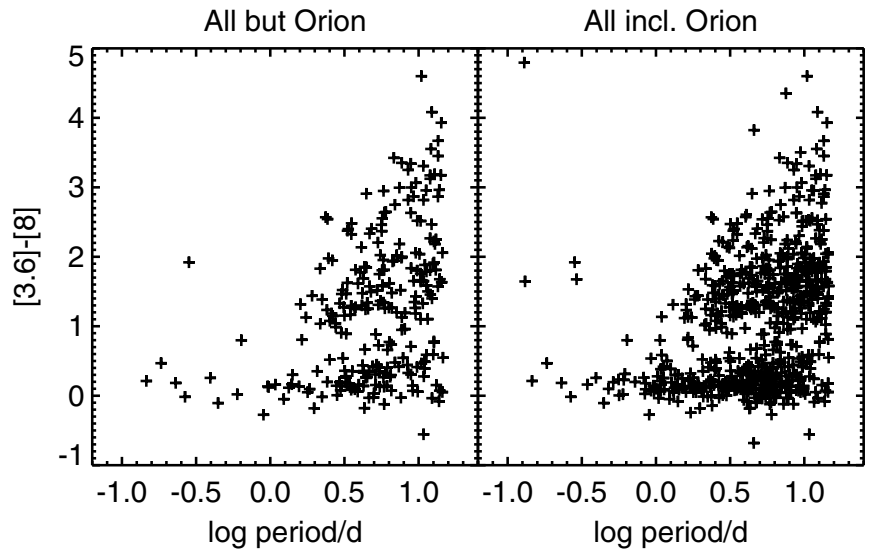

Figure 32. IR excess ([3.6]-[8]) vs. log (period in days) for objects in the YSOVAR clusters, using the periods derived from the YSOVAR data as described in the text. Left: the 11 clusters, excluding Orion; there are $\sim 250$ objects. Right: all 12 YSOVAR clusters, including Orion; there are $~ 430$ Orion objects plus the $\sim 250$ objects from left panel. The plots are similar, both to each other and to that obtained for Orion by Rebull et al. (2006), despite the fact that most of these stars are on average thought to be younger than those in Orion.

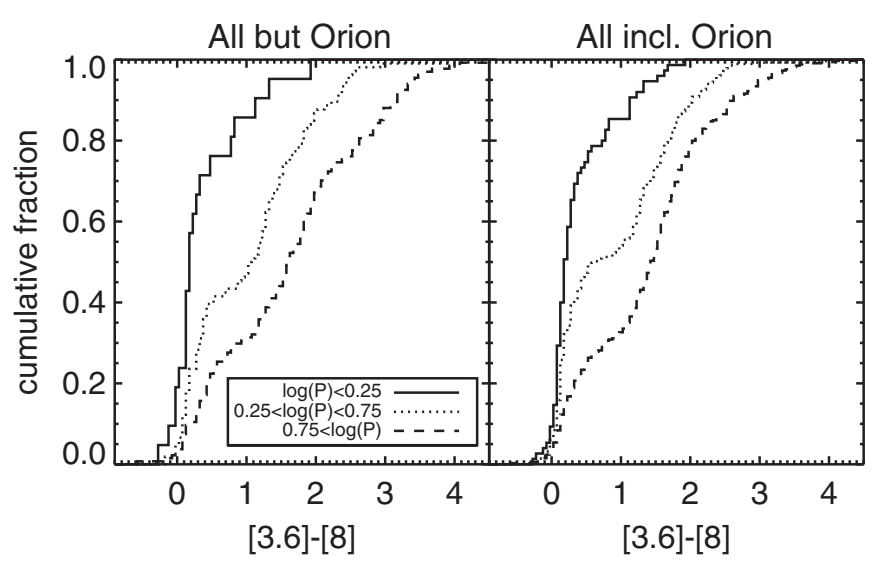

Figure 33. Cumulative distributions of IR excess ([3.6]-[8]) for objects with $\log (P) \leqslant 0.25$ (solid line), $0.25<\log (P) \leqslant 0.75$ (dotted line), and $0.75<\log (P)$ (dashed line). Left: the 11 smaller-field clusters, excluding Orion. Right: all 12 YSOVAR clusters, including Orion. The distributions are significantly different according to a K-S test.

Figure 32 shows the relationship between IR excess (specifically [3.6]-[8]) and YSOVAR-derived IR periodicity for these sources. In both cases, there is a gap near [3.6]-[8] 0.8, which divides the disk candidates (above that cutoff) from the non-disk candidates (below). There is also different behavior to the left and right of $\log (P) \sim 0.25$, or $P \sim 1.8$ days-excesses do not necessarily imply longer periods, but a star with a longer period is more likely than those with shorter periods to have an IR excess. Figure 33 shows the cumulative distributions of [3.6]-[8] for the same two panels as in Figure 32, for three different bins of $\log (P)$, divided at $\log (P)=0.25$ and 0.75 (1.78 days and 5.62 days, respectively). According to Kolmogorov-Smirnov (K-S) tests, the distributions of [3.6]-[8] are significantly different within each panel. The two distributions that are the most similar are the full (all 12 clusters) distributions for $0.25<$ $\log (P) \leqslant 0.75$, and $0.75<\log (P)$; the probability that those populations were drawn from the same distribution is $4 \%$. The probability that the populations were drawn from the same distribution for $\log (P) \leqslant 0.25$ and $0.75<\log (P)$ (again, for all 12 clusters) is $\sim 10^{-13}$; for $\log (P) \leqslant 0.25$ and $0.25<\log (P) \leqslant$ 0.75 , the probability that the populations were drawn from the 


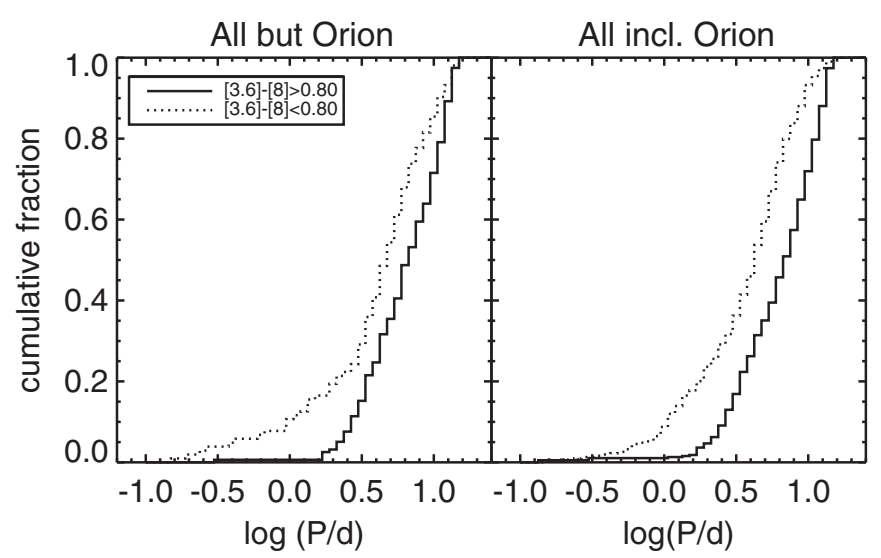

Figure 34. Cumulative distributions of $\log (P)$ for objects with [3.6]-[8] > 0.8 (solid line), and [3.6]-[8] $\leqslant 0.8$ (dotted line). Left: the 11 smaller-field clusters, excluding Orion. Right: all 12 YSOVAR clusters, including Orion. The distributions are significantly different according to a K-S test.

same distribution is $\sim 10^{-10}$. Similarly, Figure 34 shows the cumulative distributions of $\log (P)$ for the same two panels as in Figure 32, for two different bins of [3.6]-[8], divided at [3.6]$[8]=0.8$. Again, according to K-S tests, the distributions of $\log (P)$ are significantly different within each panel; the probability that either of the populations were drawn from the same distribution is $<10^{-17}$.

The plots in Figure 32 are very similar to that obtained for Orion by Rebull et al. (2006), and that by other investigators in other clusters, despite the fact that optically determined periods were used there. The periods derived from our IR YSOVAR data may be photospheric rotation rates, pulsation rates (see, e.g., Morales-Calderón et al. 2009), or inner disk rotation rates (see, e.g., Artemenko et al. 2012); on the other hand, for those clusters where there are periods available, we match the literature reasonably well, and the literature for the most part is using optical data to obtain periods. Therefore, it seems that we are, in most cases, not sampling much different locations in the star-disk system. However, an exception could be that the optical and IR observations are sampling two separate places whose movements are locked together, such as starspots on the photosphere and stellar-magnetosphere-driven disk disturbances at the corotation radius.

It is also surprising that the results for the aggregate set of clusters are so similar to that for Orion, because the clusters should be for the most part substantially younger than Orion. This could imply that disk locking may be in effect at even these young ages, or that accretion-powered stellar winds are the dominant mechanism to slow these objects. However, it is likely that we can obtain viable periods more easily for relatively unobscured stars, e.g., with these periods, we are also sampling the older end of the young star distributions in these clusters. Little is known about many of the objects outside of Orion shown here; additional study of the individual objects will help clarify matters. Individual objects will be discussed in the corresponding YSOVAR cluster paper.

\subsection{Disks Do Not Vanish or Appear}

There have been recent reports of debris disks undergoing significant short-term changes; both Meng et al. (2012) and Melis et al. (2012) report on systems that change significantly at wavelengths $>10 \mu \mathrm{m}$ over timescales of years. Our objects are much younger (a few million years rather than a few tens or hundreds of million of years) and our monitoring wavelengths are considerably shorter. However, Rice et al. (2012) also note that nine $(36 \%)$ of the stars in their sample of young stars in Cygnus OB7 (comparable in age to our sample) have a transient NIR $(J H K)$ excess. We can use this first look at our data to constrain the degree to which IR excesses in our sample vanish (or appear) on the timescales of years, namely between the cryoera observation and that of our post-cryo observations.

Irrespective of whether or not objects have been identified as variable above, we compared the cryo-era [3.6]-[4.5] color with the maximum and minimum [3.6]-[4.5] color obtained during our YSOVAR (fast-cadence) monitoring (standard set for statistics, the subset of which have measurements in both channels). Out of $\sim 11,000$ objects (cluster members as well as background objects included) for which we have [3.6]-[4.5] color light curves, there are at most 15 objects that seem to have legitimate substantial changes to the [3.6]-[4.5] color (changes of a size that might be consistent with big changes to a disk), and these are all relatively faint ([3.6] > $12 \mathrm{mag}$ ) objects. At most, two of those cases have an IR excess that appears to be possibly transient on these timescales, so at most $<0.02 \%$ frequency of occurrence. For the remaining 13 objects with plausibly real changes in color, the disk is still clearly present, but the brightness and color have changed substantially. Individual objects will be discussed in the cluster papers.

\subsection{Brightening as Likely as Fading}

In the literature (e.g., Giannini et al. 2009; Antoniucci et al. 2014), constraints have been placed on the timescales for brightening or fading by comparing how many sources are found to be getting brighter or getting fainter (e.g., for each source, given the two epochs, for how many cases is the second epoch brighter than the first, and for how many cases is the first epoch brighter than the second). If there are random fluctuations in brightness, the same number of sources should get brighter as get fainter. If, instead, there are more fading sources than brightening, then the type of variability may be characterized by a short rise and a long fall.

We can make a similar comparison among our long-term variable sample. To reduce scatter from noise, we consider only the standard set of members (Section 3.1), and consider only those objects identified as long-term variables independently in both [3.6] and [4.5] (Section 5.5). Figure 35 compares the numbers of these remaining sources that become brighter at both [3.6] and [4.5] with those that become fainter at both [3.6] and [4.5] for 11 of the clusters; errors are approximated by simple Poisson counting statistics. A significantly larger number of variables are found in Orion, with essentially equal numbers of sources getting brighter as getting fainter. For the smaller-field clusters, the numbers of sources that brighten is less consistently equal to the number of sources that fade, but there are also far fewer sources to count. Summing up the 11 smaller-field clusters, there are $107( \pm 10)$ sources that brighten and $88( \pm 9)$ sources that fade; these numbers are within $2 \sigma$ of each other, but both numbers have to be extended $\sim 1 \sigma$ toward each other. Fitting a line to the points in Figure 35, including Orion, results in a line of slope $1.05 \pm 0.12$, and an intercept of $-2.8 \pm 3.3$. This is consistent with no difference between the brightening and fading sources, but with only a $\sim 1 \sigma$ possibility that there are slightly more brightening sources. With or without Orion, there are very similar numbers brightening and fading.

In the case of several other papers in the literature, they were looking for much more significant variability than we 


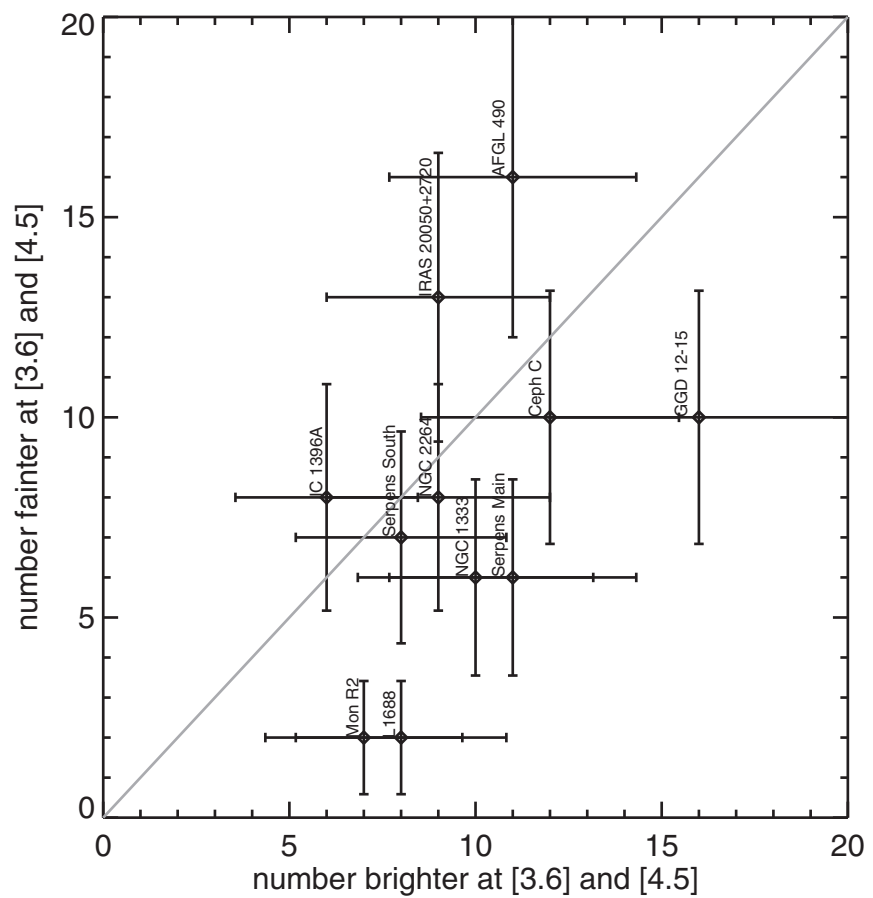

Figure 35. Plot of the number of members that become fainter vs. the number that become brighter in both [3.6] and [4.5] between the cryo epoch and the YSOVAR epoch. Error bars are approximated by Poisson statistics. The Orion point is to the far upper right, at $(156,153)$, with errors of $\sim 12$ in each direction. The gray line is the unity relation. There are similar numbers of objects that become brighter as become fainter; see the text.

are finding (e.g., FUors and EXors). They found internally consistent patterns of sharp rises and long falls in brightness. For our sample, we are apparently finding more varieties of variability, even on the long-term, such that the timescales average out over six to seven years to have no significant bias toward brightenings or fadings.

\subsection{Long-term Variability Fractions}

Having identified the long-term variables above (Section 5.5) for each cluster, we can look at the fraction of objects that are variable on the longest timescales we sample. Figure 36 shows the fraction of variables for all objects in each field (the standard set for statistics), including both members and likely field objects. The $x$ axis is our parameterization of the relative fractions of embedded sources (see Section 4.1), the ratio of Class I/total number of members. Figure 36 thus is somewhat incongruous in that the standard set of members is used in the $x$ axis, but the $y$ axis includes everything in the FOV. About 10\%-20\% of all objects with light curves are identified as variable in the long-term. However, as can be seen in the figure, the variability fraction of everything in the field is not a strong function of the Class I/total ratio- the slopes of the best-fit lines in Figure 36 are both small. (The slopes of these lines are given in the figure caption.) Calculating Pearson's correlation coefficient also suggests that there are no significant correlations shown in Figure 36. We expect that the fraction of stars that are variable should be a function of Galactic latitude (see approximate Galactic latitudes listed in Table 1), because the fraction of sources that are background/foreground stars will be higher in the Galactic plane, so the fraction of cluster members will be higher out of the Galactic plane, and since any young star (cluster member) is more likely to be variable, there should be a higher fraction of variable objects at higher Galactic latitude. Indeed, the Pearson's correlation coefficient suggests that the fraction of long-term variables for all objects in each field is strongly correlated with the absolute value of the Galactic latitude.

Figure 37 recasts Figure 36 by using the long-term variability fraction of only the standard set of members, rather than everything in the field. This time, there is a significant correlation; the higher the fraction of embedded members, the higher the fraction of long-term variables. If a cluster has more sources that are embedded and likely to be actively accreting and interacting with their circumstellar material, it also has more sources that are variable on timescales of years. The slopes of the best-fit lines shown in Figure 37 are given in the figure caption. Pearson's correlation coefficient suggests that there is a correlation here, and it is stronger in I1 than I2 (consistent with the calculated slopes).

Serpens South has the highest fraction of the most embedded sources; to test if it is providing a significant "lever arm" on this fit, we fit the remaining points omitting Serpens South, which does indeed weaken the correlation, as can be seen in the figure.

We tested the correlations seen in Figures 36 and 37 using a variety of other SED class-based parameterizations, and we found similar results - there is no significant correlation of the long-term variable fraction of all sources in the field with any SED class-based parameterization, and there is a correlation of the long-term variable fraction of the members with any parameterization chosen that uses fractions of various SED classes (or groups of classes). This correlation between the fraction of members that are variable on the longest timescales and the parameterization of "embeddedness" seems robust. We expected that young stars were more likely to be variable than field stars. Moreover, we have found that within the category of young stars, for a higher fraction of embedded sources (a higher fraction of presumably younger sources), we find a higher fraction of long-term variables. This is consistent with what has been found in individual clusters (e.g., NGC 2264 (Cody et al. 2014) and L1688 (Günther et al. 2014)).

The difference between fractions of embedded objects among these clusters is not the only potentially significant factor for this set of observations. Here, we sample timescales of $\sim 4.5$ to $\sim 7.5 \mathrm{yr}$. If the amplitude of variability of the members increases as the time baseline increases, we expect more members to be selected as variable in the long-term, and thus a higher longterm variability fraction as the time baseline increases. However, Figure 38 shows this relationship between the variability fraction and the time between epochs of observation (from Table 4). The best-fit lines and correlation coefficients are consistent with no significant effect on the variability fraction as a function of timescale sampled. Serpens South, because it was observed (indeed, discovered; Gutermuth et al. 2008b) comparatively late in the Spitzer mission, samples the shortest timescales. If the Serpens South point is omitted from the fit, the slopes become slightly steeper, but there is still no significant correlation from the correlation coefficients.

Scholz (2012), working in the $K$ band, finds that the longer one monitors a cluster, the larger the amplitude of variability, on timescales of years. That work specifically investigated the amplitude of the change in magnitude, for just one cluster at a time, and looked at the change of the range of that distribution of amplitudes as a function of time step. For the times we sampled on these longest timescales, analogous plots do not show a significant change in the median or the top quartile or the 90th 


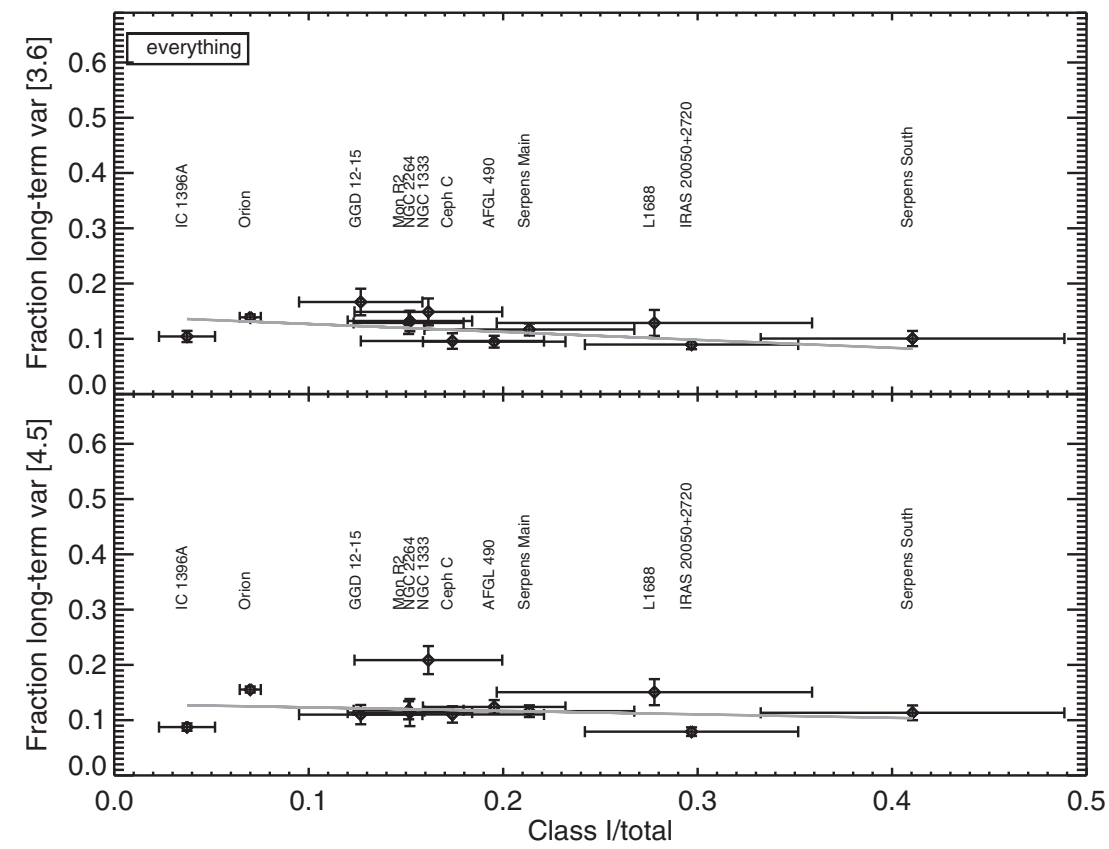

Figure 36. Fraction of long-term variables from the standard set for statistics, as a function of the ratio of Class I/total objects, for all objects in the field, for [3.6] (top) and [4.5] (bottom), for each cluster, as indicated. The range in the $y$ axis is set to match the range needed for the next figure. Error bars are calculated assuming Poisson counting statistics; Orion has by far the most sources, and so has by far the smallest error bars. The gray lines are the best-fit line, using errors in both directions for each point. The long-term variability fraction for everything in the field is relatively constant in this plot—-the slopes of these lines are, for [3.6], $-0.14 \pm 0.04$, and for [4.5], $-0.06 \pm 0.06$. Correlation coefficients suggest that there is no significant correlation here ([3.6]: $r=-0.41 ;[4.5]: r=-0.10)$.

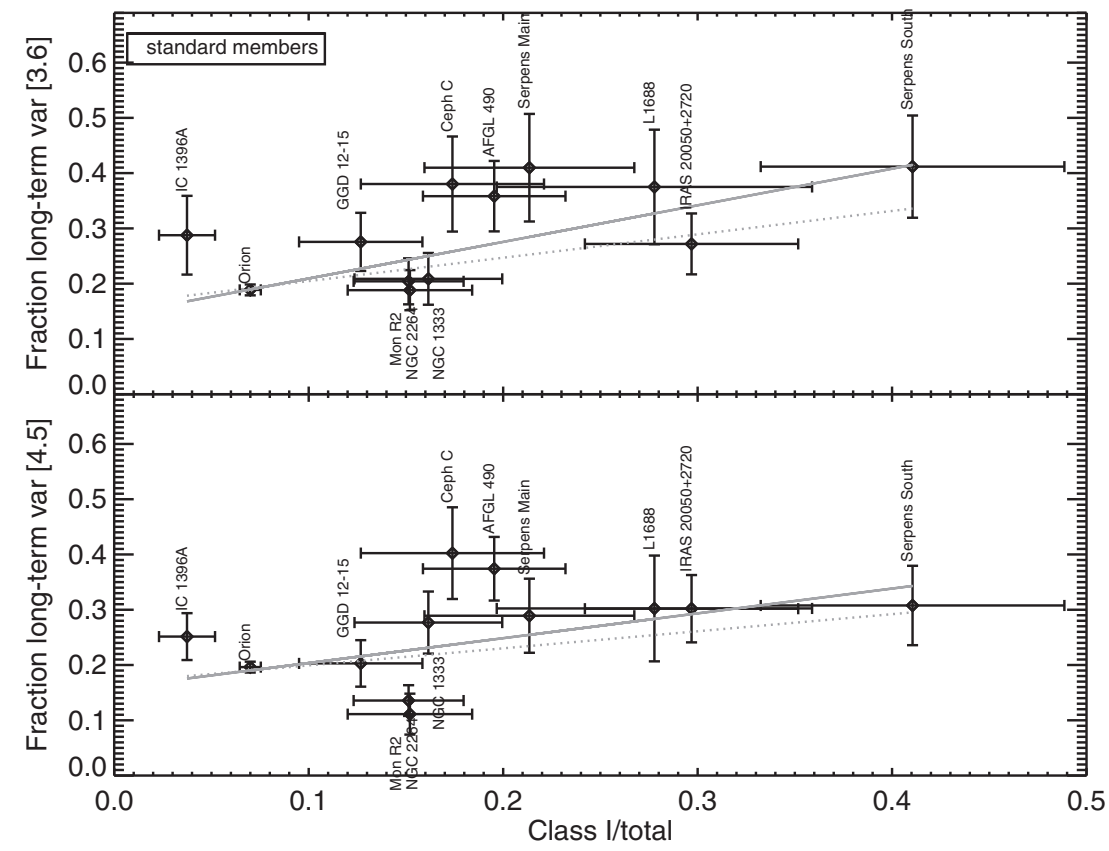

Figure 37. Similar to Figure 36, except that the $y$ axis is the fraction of long-term variable members. The slopes of the gray best-fit solid lines are, for [3.6], 0.66 \pm 0.19 , and for [4.5], $0.45 \pm 0.18$. Correlation coefficients are consistent with a correlation ([3.6]: $r=0.58 ;[4.5]: r=0.41)$. Since Serpens South has the largest fraction of embedded objects, we also tested fitting this relation omitting this point, and this does weaken the correlation. The dashed lines are these best fit values: for [3.6], $0.42 \pm 0.15$, and for [4.5], $0.31 \pm 0.14$. (Correlation coefficients are [3.6]: $r=0.45 ;$ [4.5]: $r=0.42$ ) The long-term variability fraction increases significantly as the degree of embeddedness increases (to the right).

percentile. We tried using the most stringent set of objects (only those in the standard set of members, standard set for statistics, that had light curves in both IRAC channels); we still did not find this effect. For this analysis, Scholz (2012) was only working in $\rho$ Oph, over a larger area than we were, using slightly different wavelengths, and a larger range of timescales, the maximum of which ( $\sim 2000$ days $)$ is comparable to the minimum $\Delta t(\sim 4.5 \mathrm{yr}$ $\sim 1600$ days) we consider here. These could account for the observed differences.

\section{CONCLUSIONS}

We present in this paper the data collection and reduction for the YSOVAR programs, representing nearly $800 \mathrm{hr}$ of Spitzer time studying the variability of young stars in 12 


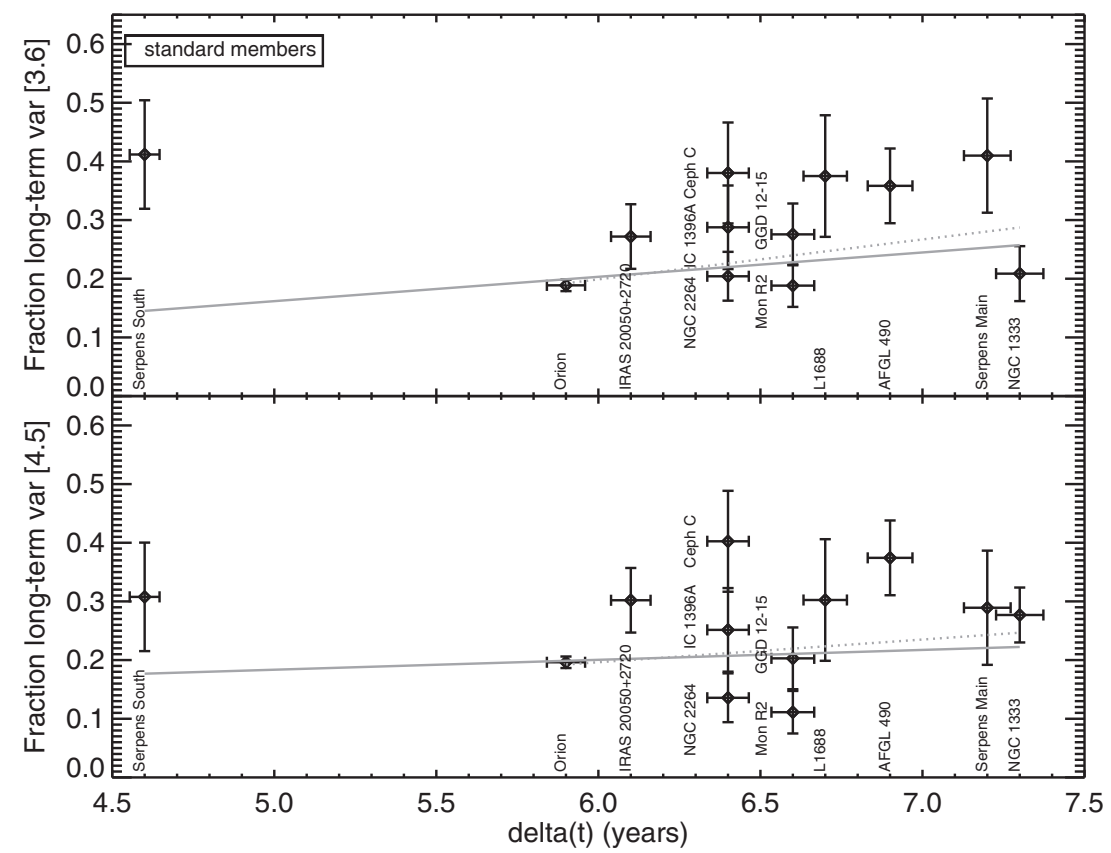

Figure 38. Long-term variable fraction for the standard set of members as a function of the time difference in years between the cryo-epoch and YSOVAR fast-cadence epoch. The slopes of the gray best-fit lines are, for [3.6], $0.04 \pm 0.02(r=-0.16)$, and for [4.5], $0.02 \pm 0.02(r=-0.0004)$. Excluding Serpens South as an outlier, the slopes of the gray dashed best-fit lines are $0.07 \pm 0.02$ and $0.04 \pm 0.02$, respectively. Correlation coefficients for these options are consistent with there being no significant correlation in either case ([3.6]: $r=0.35 ;[4.5]: r=0.23$ ). All of this evidence is consistent with no significant effect of the timescale on the fraction of long-term variables.

different clusters (AFGL 490, NGC 1333, Orion, Mon R2, GGD 12-15, NGC 2264, L1688, Serpens Main, Serpens South, IRAS 20050+2720, IC 1396A, and Ceph C). We also describe the assembly of broad collections of ancillary data for these clusters. There are $\sim 29,000$ unique objects of any sort matched to 39,000 [3.6] or [4.5] light curves in the YSOVAR data set.

The goals of the broader YSOVAR program include the following: to obtain the first extensive mid-infrared time series photometry of young stars to help reveal the structure of the inner disk region of YSOs, provide new constraints on accretion and extinction variability, assess timescales of mid-IR variability from seconds to years, identify new young eclipsing binaries, help identify new very low-mass substellar members of the surveyed clusters, constrain the short- and long-term stability of hot spots on the surfaces of YSOs, and determine rotational periods for objects too embedded for such monitoring in the optical.

In this paper, we set the stage for several planned papers. We establish here not only the data reduction approach, but also define the standard sample on which we calculate statistics (the fast cadence data, where there are at least five points per light curve), and a standard sample of members (the union of all IR-selected members and X-ray-selected members), with a provision for adding members identified in other ways (literature or variability itself).

We use three mechanisms to identify variables in the standard set for statistics (fast cadence data) - the Stetson index (calculated using both IRAC channels), the $\chi^{2}$ test (calculated for each channel individually), and searching for significant periodicity (working on light curves with at least 20 points, using primarily the LS approach, independently on [3.6], [4.5], and [3.6]-[4.5]). Based on simulations, for these YSOVAR data, we find that we are sensitive to a broad range of timescales and amplitudes. If the star is found in one of two states half the time, the Stetson test will identify it as variable in almost all cases (99\%) if the step size between states is at least three times larger than the noise level. If the star spends less than $20 \%$ of the time in either state, then there is a reasonable chance that the variability will not be found, even for larger step sizes between states, since the sampling might catch only few data points in this state. In general, we can detect periodicity with an amplitude twice the level of the noise, variability at 3-5 times the level of the noise with the Stetson test and 6-10 times the noise for single-band light curves with the $\chi^{2}$ test.

We also identified variables on the longest timescales possible using our data set, timescales of six to seven years, using a fourth method of identifying variability. By comparing measurements taken early in the Spitzer mission with the mean from our YSOVAR campaign (the standard set for statistics), we can identify those objects that have significantly changed between then and now. We show that the overall fraction of everything in each field that varies on these longest timescales is independent of the ratio of Class I/total members in each cluster. However, the fraction of members in each cluster that are variable on these longest timescales is a function of the ratio of Class I/ total members in each cluster, such that clusters that have a higher fraction of Class I objects also have a higher fraction of long-term variables. We find no dependence of the fraction of members in each cluster that are variable on these longest timescales with the time step between the observations (between the cryogenic and post-cryogenic observations). Among the most reliable of the long-term variables, we find no strong preference for brightening or fading over these timescales.

We find periods from our data in $\sim 1100$ objects, $\sim 800$ of which are in Orion alone. About 650 of those have data in both 3.6 and $8 \mu \mathrm{m}$ ( $\sim 430$ of which are in Orion), enabling us to compare [3.6]-[8] versus $\log (P)$ in a fashion similar to that found previously in Orion (Rebull et al. 2006) and NGC 2264 (Cieza $\&$ Baliber 2007). Very similar results are obtained-excesses do not necessarily imply longer periods, but a star with a longer 
period is more likely than those with shorter periods to have an IR excess. This is somewhat surprising, in that (1) the periods are determined from the IR, not the optical, as was done previously; and (2) the clusters besides Orion are thought to be substantially younger than Orion, suggesting that disk locking may be in effect at even these young ages. However, it is likely that we can obtain viable periods more easily for relatively unobscured stars, e.g., the older end of the young star distributions in these clusters. Little is known about many of the objects outside of Orion shown here; additional study of the individual objects will help clarify matters.

There have been recent reports of debris disks changing on timescales of years; both Meng et al. (2012) and Melis et al. (2012) report on systems that change significantly at wavelengths $>10 \mu \mathrm{m}$ over timescales of years. Our objects here are younger and our monitoring wavelengths are considerably shorter. Out of $\sim 11,000$ objects (cluster members as well as foreground/background objects included) for which there is an essentially simultaneous YSOVAR measurement in both [3.6] and [4.5], there are at most 15 objects that seem to have legitimate substantial changes to the [3.6]-[4.5] color (changes of a size that might be consistent with a disk appearing/ disappearing), and these are for the most part relatively faint ([3.6] > 12 mag) objects. At most, two of those cases have an IR excess that appears to be possibly transient on these timescales, so at most, $<0.02 \%$ frequency of occurrence. For the remaining 13 objects with plausibly real changes in color, the disk is still clearly present, but the brightness and color have changed substantially.

Details of individual objects of interest in each of the clusters will appear in the forthcoming YSOVAR papers.

We are grateful to the SSC scheduling staff for accommodating this complex cadence over the two years of the survey program. We thank L. Hartmann and M. Skrutskie for early help with the program and for helpful suggestions on the manuscript; we also thank N. Calvet, D. Ciardi, J. Holtzmann, J. Muzerolle, G. Rieke, K. Stapelfeldt, H. Sung, M. Werner, B. Whitney, E. Winston, and K. Wood for early help with the program. We thank T. Mazeh, L. Tal-Or, and the Wise Observatory for help in preliminary observations of Orion YSOs.

R.G. gratefully acknowledges funding support from NASA ADAP grants NNX11AD14G and NNX13AF08G and Caltech/ JPL awards 1373081, 1424329, and 1440160 in support of the Spitzer Space Telescope observing programs. H. Bouy is funded by the Ramón y Cajal fellowship program number RYC-2009-04497. This research has been funded by Spanish grants AYA2012-38897-C02-01, AYA2010-21161-C02-02, CDS2006-00070 and PRICIT-S2009/ESP-1496.

This work is based in part on observations made with the Spitzer Space Telescope, which is operated by the Jet Propulsion Laboratory, California Institute of Technology under a contract with NASA. Support for this work was provided by NASA through an award issued by JPL/Caltech. The research described in this paper was partially carried out at the Jet Propulsion Laboratory, California Institute of Technology, under contract with the National Aeronautics and Space Administration. The scientific results reported in this article are based in part on data obtained from the Chandra Data Archive including observations made by the Chandra $X$-ray Observatory and published previously in cited articles.

This research has made use of NASA's Astrophysics Data System (ADS) Abstract Service, and of the SIMBAD database, operated at CDS, Strasbourg, France. This research has made use of data products from the Two Micron All-Sky Survey (2MASS), which is a joint project of the University of Massachusetts and the Infrared Processing and Analysis Center, funded by the National Aeronautics and Space Administration and the National Science Foundation. The 2MASS data are served by the NASA/IPAC Infrared Science Archive, which is operated by the Jet Propulsion Laboratory, California Institute of Technology, under contract with the National Aeronautics and Space Administration. This research has made use of the NASA Exoplanet Archive, which is operated by the California Institute of Technology, under contract with the National Aeronautics and Space Administration under the Exoplanet Exploration Program.

\section{APPENDIX A \\ NAMING CONVENTION}

In our initial data release (MC11), we used a naming convention following the IAU naming standards using an acronym (Initial Spitzer Orion YSOVAR: ISOY) followed by the J2000 coordinates.

For the final version of our YSOVAR catalog, discussed here, the IAU-registered acronym is SSTYSV, for Spitzer Space Telescope, YSOVAR. We again follow it with the J2000 coordinates. Individual objects in this catalog need not be confirmed young stars, but simply have a light curve in this data set. Detailed data tables of cluster members for each cluster will be presented in the individual cluster papers, and it is our intention to deliver a final catalog of every object with a light curve to IRSA for general distribution.

\section{APPENDIX B \\ SED CLASSES \\ B.1. Background}

In the context of understanding the evolution of young stars from a very embedded state to a less embedded state, the community has chosen to parameterize objects based on the slope of the SED (see, e.g., Wilking et al. 2001). This classification is tied to the empirical shape of the SED. Very embedded (presumably very young) objects will have SEDs that peak at long wavelengths; as the object sheds its natal cocoon, the peak of the SED moves to shorter wavelengths. Objects with substantial circumstellar disks will emit more energy in the IR (and longer wavelengths) than in the optical. Objects with less substantial disks will have most of their energy emitted in wavelengths shorter than the NIR, but there will be additional energy contributions in the IR (and longer wavelengths) from the circumstellar dust and/or debris, i.e., the SED has an IR excess. The most embedded phase is referred to as Class 0, then proceeding (based on SED shape) through Class I (rising SED), Flat (flat SED), Class II (falling SED with an IR excess), and finally Class III SEDs, which are objects with photospheric or near-photospheric SEDs, with little or no IR excess, typically identified as young via other means such as X-rays or $\mathrm{H} \alpha$ emission. Sometimes additional classes such as transition disks are added near the end of this sequence. Classical $\mathrm{T}$ Tauri stars (CTTSs) are often identified with Class IIs, and weaklined T Tauri stars (WTTSs) are often identified with Class IIIs. Nomenclature is difficult and inconsistent across the literature; see Evans et al. (2009a) for a discussion of terminology. 
We need to establish at least an internally consistent definition of the SED classes so that we can investigate trends as a function of SED class as a proxy for age. The reliability of the translation between SED class and age has been discussed at length in the literature (and will continue to be discussed in the future); other factors such as inclination and multiplicity may play a large role. In the context of our work, we wish to establish an internally consistent approach that can be calculated for all sources in the YSOVAR fields.

\section{B.2. Definition}

In order to assemble our SEDs for each object, we include all the data described in Section 2 between $U$ and $25 \mu \mathrm{m}$. For the SED classes, only the IR bands are relevant.

In order to define an internally consistent placement of the YSOVAR objects into SED classes, in the spirit of Wilking et al. (2001), we define the near- to mid-IR $(2-24 \mu \mathrm{m})$ slope of the SED, $\alpha=d \log \lambda F_{\lambda} / d \log \lambda$, where $\alpha>0.3$ for a Class I, 0.3 to -0.3 for a flat-spectrum source, -0.3 to -1.6 for a Class II, and $<-1.6$ for a Class III. For each of the objects in our sample, we performed a simple least squares linear fit to all available photometry (only detections, not including upper or lower limits, but including archival and literature data) as observed between 2 and $24 \mu \mathrm{m}$, inclusive. We included the mean obtained from the standard set for statistics of the light curve in the SED, in addition to the cryo-era Spitzer points. (This means that there could be more than one point contributing to the fit at $3.6 \mu \mathrm{m}$, one from the cryo era and one from the mean of the YSOVAR light curves.) Formal errors (either from individual single-epoch measurements or the mean and standard deviation from the IRAC light curves) on the infrared points are so small as to not affect the fitted SED slope. However, if the mean is calculated over more of the light curve than our standard set for statistics (fast cadence) sample, the mean may be different enough, in the cases of sparse SEDs, that the class may change to an adjacent class; these cases will be noted in the cluster papers where relevant. The linear fit is performed on the observed SED, e.g., no reddening corrections are applied to the observed photometry before fitting.

In the literature, the precise definition of $\alpha$ can vary, or the distribution of slopes and classes can vary (e.g., Sung et al. 2009 lists Class I, II, II/III, pre-transition disk, transition disk categories for NGC 2264), which may result in different classifications for certain objects. Classification via our method is provided specifically to enable comparison within this paper (and to other YSOVAR papers) via internally consistent means. Our classification, since it is based on observed SED, is possible for all objects (not just those identified as YSO candidates). The formal classification definition puts no lower limit on the colors of Class III objects (thereby including those with SEDs resembling bare stellar photospheres, and allowing for other criteria such as X-ray brightness to define youth). The SED slopes and classes for all of the sources of interest will appear in the individual cluster papers. Histograms of the relative fractions of each class for the standard set of members for each cluster appear in Figure 17.

\section{B.3. Including or Ignoring the $24 \mu \mathrm{m}$ Point}

In terms of aggregate statistics over all data from all 12 clusters (members and non-members together), we can constrain the fraction of objects that change class depending on whether or not the $24 \mu \mathrm{m}$ point is included in the fit. There are about 21,000 objects with light curves over all 12 clusters for which an SED slope can be fit between 2 and $8 \mu \mathrm{m}$. Out of those $\sim 21,000$, there are only about 1760 with MIPS-24 detections (not limits), so only about $8 \%$ of the sources are affected. Admittedly, those sources that are detectable at $24 \mu \mathrm{m}$ are the ones with rising SEDs and thus are statistically more likely to be true cluster members than a source selected at random from the map. Out of the $\sim 1760$ with MIPS-24 detections, $\sim 72 \%$ of them do not change class when the $24 \mu \mathrm{m}$ point is included in the SED fit. The class bins of slopes are relatively large and thus relatively insensitive even to a point at the far red end of the SED. Of the $\sim 28 \%$ that do change class, $\sim 85 \%$ move only one step, to an adjacent class. As expected, there is a bias, when including the $24 \mu \mathrm{m}$ point, to move the objects to a more positive slope, e.g., toward the more embedded end of the sequence; of the ones that change class at all, $\sim 61 \%$ move one step earlier (toward more embedded, not necessarily in age) in the sequence, $\sim 24 \%$ of those move one step later (toward less embedded, not necessarily in age) in the sequence.

We conclude that it does not make a significant difference for the overwhelming majority of the sources if one uses the slope between 2 and $8 \mu \mathrm{m}$ or the slope between 2 and $24 \mu \mathrm{m}$. (Note that this is fitting all available points between these values, not a simple comparison of the two end points.) For any sources of interest in which the class might change depending on the inclusion of the $24 \mu \mathrm{m}$ point, they will be noted in the individual cluster papers.

\section{B.4. Comparison to $G 09$}

G09 provides placement into SED classes as part of the data presented there, and the same algorithm has been applied to our entire cryogenic-era catalog (Section 2.7). These classes are identified based on dereddened colors, and are only provided for objects thought to be young stars. Objects that are not thought to be young stars are identified as other things such as "PAH emission source"; objects missing bands such that the classification scheme cannot be run are also not classified. The SED classes we are using here are based on fits to the observed SED, not the dereddened SED, and are obtained for any source, regardless of its true underlying nature. To constrain the degree to which we might be introducing a bias by fitting the observed SED, we can compare, for some objects, the class obtained by G09 and by our mechanism above.

It is important to note that the classes provided by G09 are different than the classes we use here. G09 has Class 0, I, II, and II/III, but no Flat class. Here, we do not have Class 0; we have Class I, Flat, Class II, and Class III. Over all 12 clusters, there are $\sim 3500$ objects for which both classifications are available; $75 \%$ of those do not change class, even with the different bins that are defined. Out of the $\sim 3500$, about $14 \%$ are identified as being from a later (less embedded) class than G09, and about $11 \%$ are identified as being from an earlier (more embedded) class than G09.

G09 also dereddens the SEDs before placing them in classes; we are fitting observed SEDs. G09 does this to avoid a reddening bias toward youth - as discussed in Muench et al. (2007), the $K_{s}-$ [3.6] color can be affected by $A_{V} \sim 40$, though it takes $A_{V} \gtrsim 200$ to affect the [5.8]-[24] color. However, in our case, because we are fitting all available points between $K_{s}$ and [24], not just subtracting two points in the SED, the influence of reddening on this overall slope in the best case (where there are $K_{s}$, four bands of IRAC, and one MIPS band), simulations suggest that we would need $A_{J} \sim 11$ or $A_{V} \sim 40$ before a 
Class III object would be misclassified as a Class II. Admittedly, this is a best-case scenario, where the SED is well-populated. For a source without MIPS 24 but just $K_{S}$ through [8], we find that we need $A_{J} \sim 6$ or $A_{V} \sim 21$ before a Class III object would be misclassified as a Class II.

Our objects discussed in YSOVAR have to be bright enough to get good quality light curves at 3.6 and $4.5 \mu \mathrm{m}$, which is effectively brighter than 16th mag (see Figures 19 and 20), which means that our sources cannot generally have extremely high extinction. Out of the objects for which we have a Gutermuthderived value for $A_{K}$ and a light curve, $\sim 6 \%$ have $A_{K} \gtrsim 2$ (which corresponds to $A_{J} \gtrsim 6$ ), and $\sim 1 \%$ have $A_{K} \gtrsim 3.5$ (which corresponds to $A_{J} \gtrsim 11$ ). We conclude that any bias toward young objects is likely not substantial in our data set.

We have opted to use most often our SED class definition as described above for internal comparison and consistency. However, when discussing the Class II/Class I ratio reported in G09, we are using those classes from G09. In Sections 2.1.2 and 4.1, we discuss the Class II/Class I ratio derived in a very similar fashion as G09, using the G09 classes, but only for those objects with light curves in the standard set for statistics. This enables at least some comparison back to G09 and related works.

\section{APPENDIX C}

\section{OBSERVATION FOOTPRINTS}

In support of the discussion in Section 2.3, this Appendix includes the approximate sky coverage for a summed up image consisting of all epochs of the YSOVAR observations for each of the clusters (except for AFGL 490, which is included in the main body of the text). In each case, footprint outlines are superimposed on a reverse grayscale image of the cluster at $4.5 \mu \mathrm{m}$ obtained during the cryogenic mission. The thicker blue solid line is $3.6 \mu \mathrm{m}$ and the thicker red dashed line is $4.5 \mu \mathrm{m}$. If there is substantial field rotation during the YSOVAR campaigns, a single epoch of observation is also indicated by the thinner blue solid and red dashed lines, with the difference between the single epoch and the larger polygon due to (ecliptic latitude dependent) field rotation effects; see Section 2.3. North is up and east is to the left in each case. The distance between the farthest north and farthest south coverage here is noted in each caption. The relevant Chandra coverage in each cluster is shown as a yellow polygon.

\section{REFERENCES}

Akeson, R., Chen, X., Ciardi, D., et al. 2013, PASP, 125, 989

Alencar, S., Teixeira, P. S., Guimarães, M. M., et al. 2010, A\&A, 519, 88

Alves de Oliveira, C., \& Casali, M. 2008, A\&A, 485, 155

Anders, E., \& Grevesse, N. 1989, GeCoA, 53, 197

Antoniucci, S., Giannini, T., Le Causi, G., \& Lorenzetti, D. 2014, ApJ, 782, 51

Artemenko, S., Grankin, K., \& Petrov, P. 2012, AstL, 38, 783

Attridge, J. M., \& Herbst, W. 1992, ApJL, 398, L61

Barsony, M., Ressler, M., \& Marsh, K. 2005, ApJ, 630, 381

Benjamin, R., Churchwell, E., Babler, B. L., et al. 2003, PASP, 115, 953

Billot, N., Morales-Calderón, M., Stauffer, J., Megeath, S., \& Whitney, B. 2012, ApJ, 753, 35

Blauw, A. 1964, ARA\&A, 2, 213

Bouvier, J. 2008, A\&A, 489, L53

Bouvier, J., Cabrit, S., Fernandez, M., Martin, E. L., \& Matthews, J. M. 1993, A\&A, 272, 176

Bouvier, J., Forestini, M., \& Allain, S. 1997, A\&A, 236, 1023

Carey, S., Surace, J. A., Glaccum, W. J., et al. 2010, Proc. SPIE, 7731, 55

Carpenter, H., \& Hodapp, K. 2008, in Handbook of Star Forming Regions Vol. 1, ed. B. Reipurth (San Francisco, CA: ASP), 899

Carpenter, J., Hillenbrand, L., \& Skrutskie, M. 2001, AJ, 121, 3160 (CHS01)

Carpenter, J., Hillenbrand, L., Skrutskie, M., \& Meyer, M. 2002, AJ, 123, 1001
Choi, P. I., \& Herbst, W. 1996, AJ, 111, 283

Cieza, L., \& Baliber, N. 2007, ApJ, 671, 605

Cody, A. M., Stauffer, J., Baglin, A., et al. 2014, AJ, 147, 82

Cohen, M., \& Schwartz, R. 1976, MNRAS, 174, 137

Contreras, M., Sicilia-Aguilar, A., Muzerolle, J., et al. 2002, AJ, 124, 1585

Dawson, R., \& Fabrycky, D. 2010, ApJ, 722, 937

Dzib, S., Loinard, L., Mioduszewski, A. J., et al. 2010, ApJ, 718, 610

Eiroa, C., Djupvik, A., \& Casali, M. 2008, in Handbook of Star Forming Regions Vol. 2, The Northern Sky ASP Monograph Publications, ed. B. Reipurth (San Francisco, CA: ASP), 693

Elias, J., Lanning, H., \& Neugebauer, G. 1978, PASP, 90, 697

Evans, N., Allen, L. E., Blake, G. A., et al. 2003, PASP, 115, 965

Evans, N., Calvet, N., Cieza, L., et al. 2009a, arXiv:0901.1691

Evans, N., Dunham, M. M., Jørgensen, J. K., et al. 2009b, ApJS, 181, 321

Fazio, G., Hora, J. L., Allen, L. E., et al. 2004, ApJS, 154, 10

Fedele, D., van den Ancker, M. E., Petr-Gotzens, M. G., \& Rafanelli, P. 2007, A\&A, 472, 207

Feigelson, E., Getman, K, Townsley, L., et al. 2005, ApJS, 160, 379

Findeisen, K. 2014, PhD thesis, California Institute of Technology

Flaccomio, E., Michela, G., \& Sciortino, S. 2006, A\&A, 455, 903

Flagey, N., Boulanger, F., Verstraete, L., et al. 2006, A\&A, 453, 969

Flaherty, K. M., Muzerolle, J., Rieke, G., et al. 2013, AJ, 145, 66

Fruscione, A., McDowell, J. C., Allen, G. E., et al. 2006, SPIE Proc., 6270, $62701 \mathrm{~V}$

Getman, K., Feigelson, E., Townsley, L., et al. 2002, ApJ, 575, 354

Getman, K. V., Feigelson, E., Townsley, L., et al. 2006, ApJS, 163, 306

Getman, K. V., Feigelson, E. D., Sicilia-Aguilar, A., et al. 2012, MNRAS, 426, 2917

Getman, K. V., Flaccomio, E., Broos, P. S., et al. 2005, ApJS, 160, 319

Giannini, T., Lorenzetti, D., Elia, D., et al. 2009, ApJ, 704, 606

Giardino, G., Favata, F., Micela, G., Sciortino, S., \& Winston, E. 2007, A\&A, 463,275

Gregory, P., \& Loredo, T. 1992, ApJ, 398, 146

Grosso, N., Feigelson, E. D., Getman, K. V., et al. 2005, ApJS, 160, 530

Günther, H. M., Cody, A. M., Covey, K. R., et al. 2014, ApJ, in press (arXiv:1408.3063)

Günther, H. M., Wolk, S. J., Spitzbart, B., et al. 2012, AJ, 144, 101

Gutermuth, R., Bourke, T. L., Allen, L. E., et al. 2008a, ApJ, 673, 151

Gutermuth, R., Megeath, S. T., Myers, P. C., et al. 2009, ApJ, 184, 18 (G09)

Gutermuth, R., Megeath, S. T., Myers, P. C., et al. 2010, ApJS, 189, 352

Gutermuth, R., Myers, P. C., Megeath, S. T., et al. 2008b, ApJ, 674, 336

Haro, G. 1969, BOTT, 5, 79

Harvey, P., Merín, B., Huard, T. L., et al. 2007, ApJ, 663, 1149

Herbig, G. 1952, JRASC, 46, 222

Herbig, G. H., \& Kameswara Rao, N. 1972, ApJ, 174, 401

Herbst, W., Bailer-Jones, C. A. L., Mundt, R., Meisenheimer, K., \& Wackermann, R. 2002, A\&A, 396, 513

Herbst, W., Herbst, D., Grossman, E., \& Weinstein, D. 1994, AJ, 108, 1906

Herbst, W., LeDuc, K., Hamilton, C. M., et al. 2010, AJ, 140, 2025

Herbst, W., \& Racine, R. 1976, AJ, 81, 840

Hirota, T., Bushimata, T., Choi, Y. K., et al. 2008, PASJ, 60, 37

Hirota, T., Honma, M., Imai, H., et al. 2011, PASJ, 63, 1

Hodapp, K. 1999, AJ, 118, 1338

Imanishi, K., Koyama, K., \& Tsuboi, Y. 2001, ApJ, 557, 747

Irwin, J., Aigrain, S., Hodgkin, S., et al. 2007, MNRAS, 380, 541

Joy, A. 1945, ApJ, 102, 168

Kohno, M., Koyama, K., \& Hamaguchi, K. 2002, ApJ, 567, 423

Kovács, G., Zucker, S., \& Mazeh, T. 2002, A\&A, 391, 369

Lamm, M., Bailer-Jones, C., Mundt, R., Herbst, W., \& Scholz, A. 2014, A\&A, 417, 557

Lamm, M., Mundt, R., Bailer-Jones, C., \& Herbst, W. 2005, A\&A, 430, 1005

Lawrence, A., Warren, S. J., Almaini, O., et al. 2007, MNRAS, 379, 1599

Loinard, L., Torres, R., Mioduszewski, A., \& Rodriguez, L. 2008, ApJ, 675, 29

Loinard, L., Zapata, L., Rodríguez, L., et al. 2013, MNRAS, 430, L10

Lombardi, M., Lada, C., \& Alves, J. 2008, A\&A, 489, 143

Makidon, R., Rebull, L., Strom, S., Adams, M., \& Patten, B. 2004, AJ, 127,2228

Mamajek, E. 2008, AN, 329, 10

Masiunas, L., Gutermuth, R., Pipher, J., et al. 2012, ApJ, 752, 127

McQuillan, A., Aigrain, S., \& Mazeh, T. 2013a, MNRAS, 432, 1203

McQuillan, A., Mazeh, T., \& Aigrain, S. 2013b, ApJ, 775, 11

Megeath, T., Allen, L. E., Gutermuth, R. A., et al. 2004, ApJS, 154, 367

Megeath, T., Gutermuth, R., Muzerolle, J., et al. 2012, AJ, 144, 192

Melis, C., Zuckerman, B., Rhee, J., et al. 2012, Natur, 487, 74

Meng, H., Rieke, G., Su, K., et al. 2012, ApJ, 751, 17

Menten, K., Reid, M., Forbrich, J., \& Brunthaler, A. 2007, A\&A, 474, 515 
Morales-Calderón, M., Stauffer, J. R., Hillenbrand, L. A., et al. 2011, ApJ, 733, $50(\mathrm{MC} 11)$

Morales-Calderón, M., Stauffer, J. R., Rebull, L., et al. 2009, ApJ, 702, 1507

Morales-Calderon, M., Stauffer, J. R., Stassun, K. G., et al. 2012, ApJ, 753, 149

Moscadelli, L., Goddi, C., Cesaroni, R., Beltran, M., \& Furuya, R. 2009, ApJ, 693, 406

Muench, A. A., Lada, C. J., Luhman, K. L., Muzerolle, J., \& Young, E. 2007, AJ, 134,411

Nakajima, H., Imanishi, K., Takagi, S.-I., Koyama, K., \& Tsujimoto, M. 2003, PASJ, 55, 635

Padgett, D., Rebull, L. M., Stapelfeldt, K. R., et al. 2008, ApJ, 672, 1013

Park, B.-G., Sung, H., Bessell, M. S., \& Kang, Y. H. 2000, AJ, 120, 894

Parks, J. R., Plavchan, P., White, R., \& Gee, A. H. 2014, ApJS, 211, 3

Plavchan, P., Gee, A. H., Stapelfeldt, K., \& Becker, A. 2008a, ApJL, 684, L37

Plavchan, P., Güth, T., Laohakunakorn, N., \& Parks, J. 2013, A\&A, 554, 110

Plavchan, P., Jura, M., Kirkpatrick, J. D., Cutri, R. M., \& Gallagher, S. C. 2008b, ApJS, 175, 191

Plunkett, A., Arce, H., Corder, S., et al. 2013, ApJ, 774, 22

Raga, A., Noriega-Crespo, A., Carey, S., \& Arce, H. 2013, AJ, 145, 28

Ramirez, S., Rebull, L., Stauffer, J., et al. 2004a, AJ, 127, 2659

Ramirez, S., Rebull, L., Stauffer, J., et al. 2004b, AJ, 128, 787

Reach, W., Megeath, S. T., Cohen, M., et al. 2005, PASP, 117, 978

Rebull, L., Stapelfeldt, K. R., Evans, N. J., II, et al. 2007, ApJS, 171, 447

Rebull, L., Stauffer, J., Megeath, S., et al. 2006, ApJ, 646, 297

Rebull, L. M. 2001, AJ, 121, 1676

Rice, T., Wolk, S., \& Aspin, C. 2012, ApJ, 755, 65

Rieke, G., Young, E. T., Engelbracht, C. W., et al. 2004, ApJS, 154, 25

Rodríguez-Ledesma, M. V., Mundt, R., \& Eislöffel, J. 2009, A\&A, 502, 883

Rydgren, E., \& Vrba, F. 1983, ApJ, 267, 191

Scargle, J. D. 1982, ApJ, 263, 835

Scholz, A. 2012, MNRAS, 420, 1495

Skrutskie, M., Cutri, R. M., Stiening, R., et al. 2006, AJ, 131, 1163

Skrutskie, M., Meyer, M., Whalen, D., \& Hamilton, C. 1996, AJ, 112, 2168

Smith, R., Brickhouse, N., Leidahl, D., \& Raymond, J. 2001, ApJL, 556, L91
Spitzbart, B., Wolk, S., \& Bizunok, N. 2005, in Protostars and Planets V, ed. B Reipurth, D. Dewitt, \& K. Keil (Tucson, AZ: Univ. Arizona Press), 8518

Stassun, K., van den Berg, M., \& Feigelson, E. 2007, ApJ, 660, 704

Stassun, K., van den Berg, M., Feigelson, E., \& Flaccomio, E. 2006, ApJ, 649,914

Stassun, K. G., Mathieu, R. D., Mazeh, T., \& Vrba, F. J. 1999, AJ, 117, 2941

Stauffer, J., Cody, A. M., Baglin, A., et al. 2014, AJ, 147, 83

Stelzer, B., Flaccomio, E., Montmerle, T., et al. 2005, ApJS, 160, 557

Stetson, P. 1996, PASP, 108, 851

Straižys, V., Cernis, K., \& Bartasiute, S. 1996, BaltA, 5, 125

Straižys, V., \& Laugalys, V. 2008, in Handbook of Star Forming Regions, Vol. 1, The Northern Sky ASP Monograph Publications, ed. B. Reipurth (San Francisco, CA: ASP), 294

Stutz, A. M., Tobin, J. J., Stanke, T., et al. 2013, ApJ, 767, 36

Sung, H., Bessell, M. S., \& Lee, S.-W. 1997, AJ, 114, 2644

Sung, H., Stauffer, J., \& Bessell, M. 2009, AJ, 138, 1116

Testi, L., Palla, F., \& Natta, A. 1998, A\&AS, 133, 81

Teixeira, P., Lada, C. J., Young, E. T., et al. 2006, ApJL, 636, L45

Vrba, F., Chugainov, P. F., Weaver, W. B., \& Stauffer, J. S. 1993, AJ, 106, 1608

Vrba, F., Rydgren, A. E., Chugainov, P. F., Shakovskaia, N. I., \& Zak, D. S. 1986, ApJ, 306, 199

Walker, M. F. 1978, ApJ, 224, 546

Werner, M., Roellig, T. L., Low, F. J., et al. 2004, ApJS, 154, 1

Wilking, B., Gagné, M., \& Allen, L. 2008, in Handbook of Star Forming Regions, Vol. 2, ed. B. Reipurth (San Francisco, CA: ASP), 351

Wilking, B. A., Blackwell, J. H., Mundy, L. G., \& Howe, 1989, ApJ, 345, 257

Wilking, B. A., Bontemps, S., Schuler, R., Greene, T., \& André, P. 2001, ApJ, 551,357

Wilking, B. A., Meyer, M. R., Robinson, J. G., \& Greene, T. P. 2005, AJ, 130, 1733

Winn, J., Hamilton, C. M., Herbst, W. J., et al. 2006, ApJ, 644, 510

Winston, E., Megeath, S. T., Wolk, S. J., et al. 2007, ApJ, 669, 493

Winston, E., Megeath, S. T., Wolk, S. J., et al. 2010, AJ, 140, 266

Wolk, S., Rice, T., \& Aspin, C. 2013, ApJ, 773, 145

Zwintz, K., Hareter, M., Kuschnig, R., et al. 2009, A\&A, 502, 239 